\title{
On mesh restrictions to satisfy comparison principles, maximum principles, and the non-negative constraint: Recent developments and new results
}

\author{
Authored BY
}

\author{
M. K. Mudunuru \\ Graduate Student, University of Houston \\ K. B. Nakshatrala \\ Department of Civil \& Environmental Engineering \\ University of Houston, Houston, Texas 77204-4003. \\ phone: +1-713-743-4418, e-mail: knakshatrala@uh.edu \\ website: http://www.cive.uh.edu/faculty/nakshatrala
}

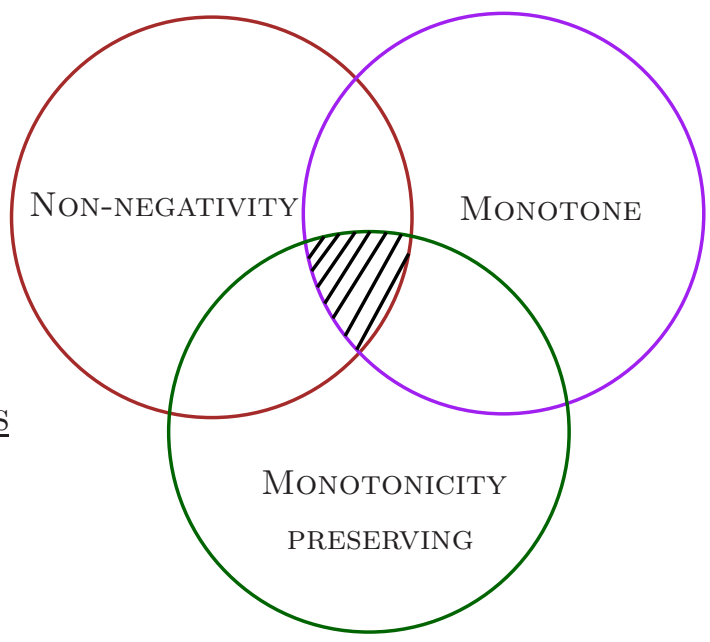

Designing a numerical methodology that possesses non-negativity, monotone, and monotonicity preserving properties is still an open problem. 


\title{
On mesh restrictions to satisfy comparison principles, maximum principles, and the non-negative constraint: Recent developments and new results
}

\author{
M. K. Mudunuru and K. B. Nakshatrala \\ Department of Civil and Environmental Engineering, University of Houston
}

\begin{abstract}
This paper concerns with mesh restrictions that are needed to satisfy several important mathematical properties - maximum principles, comparison principles, and the non-negative constraint - for a general linear second-order elliptic partial differential equation. We critically review some recent developments in the field of discrete maximum principles, derive new results, and discuss some possible future research directions in this area. In particular, we derive restrictions for a three-node triangular (T3) element and a four-node quadrilateral (Q4) element to satisfy comparison principles, maximum principles, and the non-negative constraint under the standard single-field Galerkin formulation. Analysis is restricted to uniformly elliptic linear differential operators in divergence form with Dirichlet boundary conditions specified on the entire boundary of the domain. Various versions of maximum principles and comparison principles are discussed in both continuous and discrete settings. In the literature, it is well-known that an acute-angled triangle is sufficient to satisfy the discrete weak maximum principle for pure isotropic diffusion. Herein, we show that this condition can be either too restrictive or not sufficient to satisfy various discrete principles when one considers anisotropic diffusivity, advection velocity field, or linear reaction coefficient. Subsequently, we derive appropriate restrictions on the mesh for simplicial (e.g., T3 element) and non-simplicial (e.g., Q4 element) elements. Based on these conditions, an iterative algorithm is developed to construct simplicial meshes that preserves discrete maximum principles using existing open source mesh generators. Various numerical examples based on different types of triangulations are presented to show the pros and cons of placing restrictions on a computational mesh. We also quantify local and global mass conservation errors using representative numerical examples, and illustrate the performance of metric-based meshes with respect to mass conservation.
\end{abstract}

\section{INTRODUCTION, MOTIVATION, AND CONTEMPORARY ADVANCEMENTS}

Diffusion-type equations are commonly encountered in various branches of engineering, sciences, and even in economics $[\mathbf{1}-\mathbf{3}]$. These equations have been well-studied in Applied Mathematics, and several properties and a priori estimates have been derived [4]. Numerous numerical formulations have been proposed and their performance has been analyzed both theoretically and numerically [5]. Several sophisticated software packages, such as ABAQUS [6], ANSYS [7], COMSOL [8], and

Key words and phrases. Mesh restrictions; anisotropic $\mathcal{M}$-uniform meshes; angle conditions; comparison principles; maximum principles; non-negative solutions; elliptic equations; local and global species balance. 
MATLAB's PDE Toolbox [9], have been developed to solve these types of equations. Special solvers for solving the resulting discrete equations have also been proposed and studied adequately [10].

This paper is concerned with numerical solutions for anisotropic advection-diffusion-reaction equations. Despite the aforementioned advances, it should be noted that a numerical solution always loses some mathematical properties that the exact solution possesses. In particular, the aforementioned software packages and popular numerical formulations do not satisfy the so-called discrete comparison principles (DCPs), discrete maximum principles (DMPs), and the non-negative constraint (NC). For example, consider the numerical simulation for pure anisotropic diffusion equation in an L-shaped domain with multiple holes using the commercial software package ABAQUS.

Numerical simulations are performed based on various unstructured finite element meshes (see Figure 1, which is to the scale), using the following popular anisotropic diffusivity tensor from hydrogeological and subsurface flow literature [11]:

$$
\mathbf{D}(\mathbf{x})=\mathbf{R D}_{\text {eigen }} \mathbf{R}^{\mathrm{T}}
$$

where $\mathbf{D}_{\text {eigen }}$ is a diagonal matrix comprised of the eigenvalues of $\mathbf{D}(\mathbf{x})$. The corresponding principal eigenvectors are the column entries in the orthogonal matrix $\mathbf{R}$. The expressions for $\mathbf{D}_{\text {eigen }}$ and $\mathbf{R}$ are assumed as follows:

$$
\begin{aligned}
\mathbf{R} & =\left(\begin{array}{cc}
\cos (\theta) & -\sin (\theta) \\
\sin (\theta) & \cos (\theta)
\end{array}\right) \\
\mathbf{D}_{\text {eigen }} & =\left(\begin{array}{cc}
d_{\text {max }} & 0 \\
0 & d_{\text {min }}
\end{array}\right)
\end{aligned}
$$

Herein, $d_{\max }$ and $d_{\min }$ correspond to the maximum and minimum eigenvalues. $\theta$ corresponds to the angle of orientation of the eigenvector coordinate system. It should be noted that these eigenvalues have physical significance and are related to the transverse and longitudinal diffusivities in the eigenvector coordinate system. Diffusion process is simulated based on equations (2.1a) $-(2.1 \mathrm{~b})$. We assume $\mathbf{v}(\mathbf{x})=\mathbf{0}, \alpha(\mathbf{x})=0$, and $f(\mathbf{x})=0$. The prescribed concentration on the sides of the L-shaped domain is equal to zero. Correspondingly, the concentration on the perimeter of the holes are set to be equal to one. The values of $d_{\max }, d_{\min }$, and $\theta$ for $\mathbf{D}(\mathbf{x})$ given by the equations (1.1)-(1.3) are assumed to be equal to 1000,1 , and $\frac{\pi}{3}$.

Very fine triangular (where the total number of nodes and mesh elements are equal to 86326 and 169453) and quadrilateral (where the total number of nodes and mesh elements are equal to 91778 and 90625) meshes are used to perform ABAQUS numerical simulations. The concentration profile obtained is shown in Figure 2. In this figure, we have not shown the concentration contour using four-node quadrilateral mesh, as it is almost identical to that of the contour obtained by employing three-node triangular mesh. The white area within the L-shaped domain with multiple holes represents the region in which the numerical value of concentration is negative and also exceeds the maximum. To be precise, in the case of triangular mesh, $2.22 \%$ and $3.92 \%$ of the nodes have violated the non-negative and maximum constraints. Correspondingly, the minimum and maximum values for concentration obtained are -0.0238 and 1.0076 . These are considerably far away from the possible values, which are between 0 and 1 . Similarly, for quadrilateral mesh, these values are slightly lower. Quantitatively, these are around $2.17 \%$ and $3.75 \%$. But the minimum and maximum values of concentration (-0.0287 and 1.0086) are slightly higher than that of the triangular mesh. Additionally, it is evident that more than $6 \%$ of the nodes have unphysical negative values for the concentration. There are nodes for which the concentration exceeded the maximum constraint. 
Furthermore, from Figures 3 and 4, it is evident that these values do not decrease with mesh refinement. In general, there are three possible routes to overcome such limitations and satisfy DCPs, DMPs, and NC; which we shall describe below.

1.1. Strategy - I: Mesh restrictions. The first approach is to place restrictions on the mesh to meet maximum principles and the non-negative constraint. For isotropic homogeneous diffusivity, Ciarlet and Raviart [12] have shown that numerical solutions based on the single-field Galerkin finite element formulation, in general, does not converge uniformly. It should however be noted that the single-field Galerkin formulation is a converging scheme. Ciarlet and Raviart have also shown that a sufficient condition for single-field Galerkin formulation to converge uniformly for isotropic diffusion is to employ a well-centered three-node triangular element mesh with low-order interpolation.

The obvious advantage of this approach is that one can use the single-field Galerkin formulation without any modification. The drawback is that an appropriate computational mesh may not exist because of the required restrictions on the shape and size of the finite element. For example, it is not an easy task (sometimes it not possible) to generate a well-centered triangular mesh for any given two-dimensional domain [13]. Note that requiring a mesh to be well-centered is a more stringent than requiring the mesh to be Delaunay. In fact, a well-centered mesh is Delaunay but the converse need not be true.

In scientific literature, there are numerous commercial and non-commercial mesh generators that produce premium quality structured and unstructured meshes for various complicated domains. For instance, the survey paper by Owen [14] accounts for more than 70 unstructured mesh generation software products. But it should be emphasized that Owen [14] rarely mentions about non-obtuse, acute, and anisotropic $\mathcal{M}$-uniform mesh generators. However, it is evident from the above discussion that these types of meshes have a profound impact on solving various important physical problems related to diffusion-type equations. In recent years, there has been considerable effort in developing such types of mesh generators. For example, some open source meshing software packages which are relevant to mesh restrictions methodology are as follows:

- Non-obtuse and acute triangulations in 2D: aCute [15-17] (a meshing software, which is based on Triangle [18])

- Anisotropic $\mathcal{M}$-uniform triangulations in 2D: BAMG [19] in FreeFem $++[\mathbf{2 0}, \mathbf{2 1}]$, BL2D [22]

- Anisotropic $\mathcal{M}$-uniform triangulations in 3D: Mmg3d [23]

- Locally uniform anisotropic Delaunay meshes (surface, 2D, and 3D): CGALmesh [24-27]

However, the use of these mesh generators in the area of numerical analysis and engineering, in particular, to construct mesh restrictions for diffusion-type equations to satisfy DCPs, DMPs, and NC is hardly known. Recently, Huang and co-workers [28-30] used BAMG to generate anisotropic simplicial meshes to satisfy various discrete properties for linear advection-diffusion-reaction equations. But in their research works, the computational domains under consideration are uncomplicated.

1.2. Strategy - II: Non-negativity, monotone, and monotonicity preserving formulations. The second approach is mainly concerned with developing new innovative numerical methodologies based on certain physical and variational principles, so that they satisfy DCPs, DMPs, and NC. Broadly, these methods can be classified into the following three categories:

- Non-negative formulations: A numerical method belongs to the class of non-negative formulations if the resulting numerical solution satisfies certain DMPs and NC. 
- Monotone formulations: A numerical method is said to be monotone if the resulting numerical solution satisfies certain DMPs, DCPs, and NC.

- Monotonicity preserving formulations: A numerical formulation is said to be monotonicity preserving if the resulting numerical solution does not exhibit spurious oscillations within itself.

It needs to be emphasized that a non-negative formulation need not satisfy monotone conditions, a monotone numerical method need not be monotonicity preserving, and vice-versa. It is still an open research problem to develop a numerical formulation that meets all the aforementioned properties.

Some popular formulations and notable research works in this direction are finite difference schemes (FDS) $[\mathbf{3 1}, \mathbf{3 2}]$, mimetic finite difference methods (MFDM) $[\mathbf{3 3}, \mathbf{3 4}]$, finite volume methods (FVM) [35-37], and finite element methods (FEM) [38-41]. It should be noted that most of these techniques are inherently non-linear. For example, the optimization-based finite element methodologies proposed by Nakshatrala and co-workers $[\mathbf{1 1}, \mathbf{4 1}-\mathbf{4 3}]$ enforce the desirable properties as explicit constraints. This is achieved by constructing variationally consistent constrained minimization problems for various numerical formulations. But one should note that this comes with an additional computational cost. However, Nakshatrala et al. [11] have shown through numerical experiments that the additional computational cost is less than $10 \%$.

1.3. Strategy - III: Post-processing methods. The third approach is about post-processing (PP) based methods. In literature, there are various types of PP methods, which can be used to recover certain discrete properties for diffusion-type equations. Some of the notable research works in this direction include:

- Local and global remapping/repair methods $[\mathbf{4 4 , 4 5 ]}$

- Constrained monotonic regression based methods [46]

- Cutoff methods (also known as the clipping methods) $[\mathbf{4 7}, \mathbf{4 8}]$

- A combination of remapping/repair methods and cutoff methods $[49,50]$

We shall now give a brief description of the pros and cons of these methods. Nevertheless, it is very difficult to apply these techniques to recover DCPs, DMPs, and NC for higher-order FEM methods, as the shape functions can change their sign within the element. In addition, most of the above methods do not have a variational basis.

The remapping/repair techniques proposed by Shashkov and co-workers are designed to improve the quality of the numerical solutions, so that they satisfy some discrete properties. Even though these are efficient, conservative, linearity and bound preserving interpolation algorithms, it should be emphasized that they are mesh dependent. In addition, application of such algorithms to anisotropic diffusion equations to satisfy DMPs and NC are seldom $[\mathbf{4 9}, \mathbf{5 0}]$.

The post-processing procedure based on a constrained monotonic regression problem proposed by Burdakov et al. [46] is locally conservative, bound preserving, and monotonicity recovering method. This is a constrained optimization-based PP method, wherein one needs to specify various constraints in order to fulfill certain discrete properties. It is applicable to FDS, FVM, and FEM. But in the case of FEM, this PP method is valid only for linear and multi-linear shape functions. In order to construct appropriate constraints for the optimization problem, one needs to know a prior information on the lower bounds, upper bounds, and monotonicity of the numerical solution for the physical problem. In general, obtaining the qualitative and quantitative nature of the solution to a given physical problem is not always possible. If such information on the monotonicity, lower and upper bounds for the numerical solution is not available, then this methodology reduces to the 
standard clipping procedure. In addition, one should note that it is not always possible to satisfy DCPs using this constrained monotonic regression algorithm. For instance, a counterexample similar to the research work by Nakshatrala et al. (see [11, Section 4]) can be constructed to show that it does not satisfy a DCP.

Finally, we would like to emphasize that a posterior cutoff analysis is a variational crime. In general, this method is neither conservative nor satisfies DMPs and DCPs. The primary objective of this method is to cut off the values of a numerical solution if it is less than a given number (which is the cutoff value). Hence, it is called the cutoff method. In the case of highly anisotropic diffusion problems and for distorted meshes, this method predicts erroneous numerical results $[\mathbf{1 1}, \mathbf{4 2}]$. By specifying the cutoff value to be zero, it is always guaranteed to satisfy NC through this methodology. In addition, if the nature of the solution is known a prior, then one can also prevent undershooting and overshooting of the numerical solution by chopping off those values.

1.4. Main contributions and an outline of this paper. Herein, we shall focus on the first approach of placing restrictions on the mesh to meet desired mathematical properties. We shall derive sufficient conditions for restrictions on the three-node triangular and four-node quadrilateral finite elements to meet comparison principles, maximum principles, and the non-negative constraint in the case of heterogeneous anisotropic advection-diffusion-reaction (ADR) equations. The notable contributions of this paper are as follows:

(i) We provide an in-depth review of various versions of comparison principles, maximum principles, and the non-negative constraint in the continuous setting.

(ii) We derive necessary and sufficient conditions on the coefficient (i.e., the "stiffness") matrix to satisfy discrete weak and strong comparison principles.

(iii) A relationship between various discrete principles within the context of mesh restrictions, numerical formulations, and post-processing methods is presented.

(iv) We propose an iterative method to generate simplicial meshes that satisfy discrete properties using open source mesh generators such as BAMG, FreeFem++, and Gmsh.

(v) Different types of non-dimensional quantities are proposed for anisotropic diffusivity, which are variants of the standard Péclet and Damköhler numbers. These quantities are extremely useful in numerical simulations and have not been discussed in the literature.

(vi) Lastly, several realistic numerical examples are presented to corroborate the theoretical findings as well as to show the importance of preserving discrete principles.

The remainder of this paper is organized as follows. In Section 2, we present the governing equations for a general linear second-order elliptic equation and discuss associated mathematical principles: comparison principles, maximum principles, and the non-negative constraint. Section 3 provides several important remarks on the continuous and discrete properties of elliptic equations. In Section 4, we shall derive mesh restrictions for the three-node triangular element and the rectangular element to meet the discrete versions of maximum principles, comparison principles, and the non-negative constraint. Finally, conclusions are drawn in Section 5.

We will denote scalars by lower case English alphabet or lower case Greek alphabet (e.g., concentration $c$ and density $\rho$ ). We will make a distinction between vectors in the continuum and finite element settings. Similarly, a distinction will be made between second-order tensors in the continuum setting versus matrices in the discrete setting. The continuum vectors are denoted by lower case boldface normal letters, and the second-order tensors will be denoted using upper case boldface normal letters (e.g., vector $\mathbf{x}$ and second-order tensor $\mathbf{D}$ ). In the finite element context, 
we shall denote the vectors using lower case boldface italic letters, and the matrices are denoted using upper case boldface italic letters (e.g., vector $\boldsymbol{f}$ and matrix $\boldsymbol{K}$ ). Other notational conventions adopted in this paper are introduced as needed.

\section{LINEAR SECOND-ORDER ELLIPTIC EQUATION AND ASSOCIATED MATHEMATICAL PRINCIPLES}

Let $\Omega \subset \mathbb{R}^{n d}$ be a open bounded domain, where " $n d$ " denotes the number of spatial dimensions. The boundary of the domain is denoted by $\partial \Omega$, which is assumed to be piecewise smooth. Mathematically, $\partial \Omega:=\bar{\Omega}-\Omega$, where a superposed bar denotes the set closure. A spatial point is denoted by $\mathbf{x} \in \bar{\Omega}$. The gradient and divergence operators with respect to $\mathbf{x}$ are, respectively, denoted by $\operatorname{grad}[\bullet]$ and $\operatorname{div}[\bullet]$. Let $c(\mathbf{x})$ denote the concentration field. We assume that Dirichlet boundary condition is prescribed (i.e., the concentration is prescribed) on the entire boundary.The remainder of this paper deals with the following boundary value problem, which is written in terms of a general linear second-order differential operator in divergence form:

$$
\begin{aligned}
& \mathcal{L}[c]:=-\operatorname{div}[\mathbf{D}(\mathbf{x}) \operatorname{grad}[c(\mathbf{x})]]+\mathbf{v}(\mathbf{x}) \cdot \operatorname{grad}[c(\mathbf{x})]+\alpha(\mathbf{x}) c(\mathbf{x})=f(\mathbf{x}) \quad \text { in } \Omega \\
& c(\mathbf{x})=c^{\mathrm{p}}(\mathbf{x}) \quad \text { on } \partial \Omega
\end{aligned}
$$

where $\mathcal{L}$ denotes the second-order linear differential operator, $f(\mathbf{x})$ is the prescribed volumetric source, $\alpha(\mathbf{x})$ is the linear reaction coefficient, $\mathbf{v}(\mathbf{x})$ is the velocity vector field, $\mathbf{D}(\mathbf{x})$ is the anisotropic diffusivity tensor, and $c^{\mathrm{p}}(\mathbf{x})$ is the prescribed concentration. Physics of the problem demands that the diffusivity tensor (which is a second-order tensor) be symmetric. That is,

$$
\mathbf{D}^{\mathrm{T}}(\mathbf{x})=\mathbf{D}(\mathbf{x}) \quad \forall \mathbf{x} \in \Omega
$$

REMARK 2.1. In mathematical analysis, the divergence form is a suitable setting for energy methods. However, some studies on maximum principles do employ the non-divergence form, which can be written as follows:

$$
\mathcal{L}[c]=\sum_{i, j=1}^{n d}(\mathbf{P})_{i j} \frac{\partial^{2} c}{\partial x_{i} \partial x_{j}}+\sum_{i=1}^{n d}(\mathbf{q})_{i} \frac{\partial c}{\partial x_{i}}+r(\mathbf{x}) c
$$

where the coefficient $(\mathbf{P})_{i j},(\mathbf{q})_{i}$, and $r(\mathbf{x})$, can be related to the physical quantities such as the diffusivity tensor, velocity field, and linear reaction coefficient. It should be, however, noted that the non-divergence form exists irrespective of differentiability of the diffusivity tensor. If $\mathbf{D}(\mathbf{x})$ is continuously differentiable, then there exists a one-to-one correspondence between the divergence form and the non-divergence form. In such cases, the operator $\mathcal{L}$ in the divergence form given by equation (2.1a) can be put into the following non-divergence form [51, Chapter 6]:

$$
\mathcal{L}[c]=-\mathbf{D}(\mathbf{x}) \cdot \operatorname{grad}[\operatorname{grad}[c(\mathbf{x})]]+(\mathbf{v}(\mathbf{x})-\operatorname{div}[\mathbf{D}(\mathbf{x})]) \cdot \operatorname{grad}[c(\mathbf{x})]+\alpha(\mathbf{x}) c(\mathbf{x})
$$

where we have used the following identity in combination with equation (2.2) to obtain equation $(2.4)$

$$
\operatorname{div}\left[\mathbf{D}^{\mathrm{T}}(\mathbf{x}) \operatorname{grad}[c(\mathbf{x})]=\mathbf{D}(\mathbf{x}) \cdot \operatorname{grad}[\operatorname{grad}[c(\mathbf{x})]]+\operatorname{div}[\mathbf{D}(\mathbf{x})] \cdot \operatorname{grad}[c(\mathbf{x})]\right.
$$

Based on the nature of the coefficients and connectedness of the physical domain, different versions of maximum and comparison principles exist in the mathematical literature [51-53]. As stated earlier in this paper, we shall restrict ourselves to the boundary value problem given by the equations (2.1a)-(2.1b). Further analysis pertaining to Neumann boundary conditions and mixed 
boundary conditions within the context of maximum principles, comparison principles, and the non-

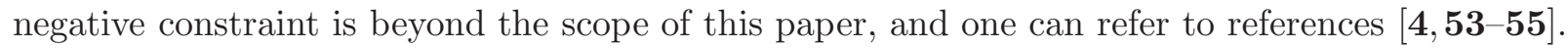

We shall say that the operator $\mathcal{L}$ is elliptic at a point $\mathbf{x} \in \Omega$ if

$$
0<\lambda_{\min }(\mathbf{x}) \boldsymbol{\xi} \cdot \boldsymbol{\xi} \leq \boldsymbol{\xi} \cdot \mathbf{D}(\mathbf{x}) \boldsymbol{\xi} \leq \lambda_{\max }(\mathbf{x}) \boldsymbol{\xi} \cdot \boldsymbol{\xi} \quad \forall \boldsymbol{\xi} \in \mathbb{R}^{n d} \backslash\{\mathbf{0}\}
$$

where $\lambda_{\min }(\mathbf{x})$ and $\lambda_{\max }(\mathbf{x})$ are, respectively, the minimum and maximum eigenvalues of $\mathbf{D}(\mathbf{x})$. The operator $\mathcal{L}$ is said to be strictly elliptic if there exists a constant $\lambda_{0}$, such that

$$
0<\lambda_{0} \leq \lambda_{\min }(\mathbf{x}) \quad \forall \mathbf{x} \in \Omega
$$

and uniformly elliptic if

$$
0<\frac{\lambda_{\max }(\mathbf{x})}{\lambda_{\min }(\mathbf{x})}<+\infty \quad \forall \mathbf{x} \in \Omega
$$

In the studies on maximum principles, it is common to impose the following restrictions on the velocity field $\mathbf{v}(\mathbf{x})$ and the linear reaction coefficient $\alpha(\mathbf{x})$ :

$$
\begin{aligned}
& \alpha(\mathbf{x}) \geq 0 \quad \forall \mathbf{x} \in \Omega \\
& \alpha(\mathbf{x})-\frac{1}{2} \operatorname{div}[\mathbf{v}(\mathbf{x})] \geq 0 \quad \forall \mathbf{x} \in \Omega \\
& 0 \leq \frac{\left|(\mathbf{v}(\mathbf{x}))_{i}\right|}{\lambda_{\min }(\mathbf{x})} \leq \beta_{0}<+\infty \quad \forall \mathbf{x} \in \Omega \quad \text { and } \quad \forall i=1, \cdots, n d
\end{aligned}
$$

where $\beta_{0}$ is a bounded non-negative constant. If $(\mathbf{D})_{i j}$ and $(\mathbf{v})_{i}$ are continuous in $\Omega$, then the operator $\mathcal{L}$ is uniformly elliptic for any bounded subdomain $\Omega^{\prime} \subset \subset \Omega$ (which means that $\Omega^{\prime}$ is compactly embedded in $\Omega$ ) and the condition given in equation $(2.9 \mathrm{c})$ holds. The restrictions given in equation (2.9b) can be relaxed in some situations (e.g., see references $[\mathbf{2 9 , 3 0 ]}$ ). But the constraint on $\alpha(\mathbf{x})$ given by equation (2.9a) cannot be relaxed. If $\alpha(\mathbf{x})<0$, then equation (2.1a) is referred to as an Helmholtz-type equation, which does not possess a maximum principle. From the theory of partial differential equations, it is well-known that the aforementioned boundary value problem given by equations (2.1a)-(2.1b) satisfies the so-called (weak and strong) comparison principles, (weak and strong) maximum principles, and the non-negative constraint. For future reference and for completeness, we shall briefly outline the main results. For a more detailed mathematical treatment, one could consult references $[\mathbf{4}, \mathbf{5 1}, \mathbf{5 2}]$.

TheOREM 2.2 (Continuous weak and strict weak maximum principles). Let $\mathcal{L}$ be a uniformly elliptic operator satisfying the conditions given by equations (2.9a)-(2.9c). In addition, let $\mathbf{D}(\mathbf{x})$ be continuously differentiable. Suppose that $c(\mathbf{x}) \in C^{2}(\Omega) \cap C^{0}(\bar{\Omega})$ satisfies the differential inequality $\mathcal{L}[c] \leq 0$ in $\Omega$, then the maximum of $c(\mathbf{x})$ in $\bar{\Omega}$ is obtained on $\partial \Omega$. That is, $c(\mathbf{x})$ possesses the weak maximum principle (wMP), which can be written as follows:

$$
\max _{\mathbf{x} \in \bar{\Omega}}[c(\mathbf{x})] \leq \max \left[0, \max _{\mathbf{x} \in \partial \Omega}[c(\mathbf{x})]\right]
$$

Moreover, if $\alpha(\mathbf{x})=0$, then we have the strict weak maximum principle (WMP):

$$
\max _{\mathbf{x} \in \bar{\Omega}}[c(\mathbf{x})]=\max _{\mathbf{x} \in \partial \Omega}[c(\mathbf{x})]
$$

Proof. For a proof, see references $[\mathbf{5 1 , 5 2}]$. 
TheOREM 2.3 (Continuous strong and strict strong maximum principles). Let the domain $\Omega$ be simply connected. Given that $c(\mathbf{x})$ satisfies $\mathrm{wMP}$ and the conditions given in Theorem 2.2, then $c(\mathbf{x})$ cannot attain an interior non-negative maximum in $\bar{\Omega}$ unless it is a constant. This means that, $c(\mathbf{x})$ possesses the strong maximum principle (sMP) if the following hold:

$$
\max _{\mathbf{x} \in \Omega}[c(\mathbf{x})]=\max _{\mathbf{x} \in \bar{\Omega}}[c(\mathbf{x})]=m \geq 0 \quad \Rightarrow \quad c(\mathbf{x}) \equiv m \quad \text { in } \bar{\Omega}
$$

Moreover, if $\alpha(\mathbf{x})=0$ and $c(\mathbf{x})$ satisfies WMP, then we have the strict strong maximum principle (SMP) given as follows:

$$
\max _{\mathbf{x} \in \Omega}[c(\mathbf{x})]=\max _{\mathbf{x} \in \bar{\Omega}}[c(\mathbf{x})]=m \quad \Rightarrow \quad c(\mathbf{x}) \equiv m \quad \text { in } \bar{\Omega}
$$

Proof. For a proof, see references $[\mathbf{5 1 , 5 2}]$.

TheOREM 2.4 (Continuous weak and strong comparison principles). Let $c_{1}(\mathbf{x}), c_{2}(\mathbf{x}) \in$ $C^{2}(\Omega) \cap C^{0}(\bar{\Omega})$. Suppose $\mathcal{L}$ be a uniformly elliptic operator satisfying the conditions given by the equations $(2.9 \mathrm{a})-(2.9 \mathrm{c})$. Then $\mathcal{L}$ is said to possess

- the weak comparison principle $(\mathrm{wCP})$ if $c_{1}(\mathbf{x})$ and $c_{2}(\mathbf{x})$ satisfies $\mathrm{wMP}, \mathcal{L}\left[c_{1}\right] \leq \mathcal{L}\left[c_{2}\right]$ in $\Omega$, and $c_{1}(\mathbf{x}) \leq c_{2}(\mathbf{x})$ on $\partial \Omega$, then the following holds:

$$
c_{1}(\mathbf{x}) \leq c_{2}(\mathbf{x}) \quad \forall \mathbf{x} \in \bar{\Omega}
$$

- the strong comparison principle $(\mathrm{sCP})$ if $c_{1}(\mathbf{x})$ and $c_{2}(\mathbf{x})$ satisfies $\mathrm{sMP}, \mathcal{L}\left[c_{1}\right]<\mathcal{L}\left[c_{2}\right]$ in $\Omega$, and $c_{1}(\mathbf{x}) \leq c_{2}(\mathbf{x})$ on $\partial \Omega$, then the following holds:

$$
c_{1}(\mathbf{x})<c_{2}(\mathbf{x}) \quad \forall \mathbf{x} \in \Omega
$$

PROOF. For proof, see reference [52].

Numerical formulations based on the finite element method, finite volume method, and finite difference method exist to solve the boundary value problem given by the equations (2.1a)-(2.1b). It is well-known that the framework offered by the finite element method is particularly attractive in obtaining accurate numerical results for elliptic partial differential equations. In particular, the single-field Galerkin formulation is a very popular finite element formulation. In this paper, we shall use the single-field Galerkin formulation to derive mesh restrictions. It should be, however, noted that restrictions imposed on a mesh may alter if an alternate numerical formulation is employed. But the overall procedure presented in this paper can be employed to derive mesh restrictions for other numerical formulations.

2.1. Single-field Galerkin formulation. Let us define the following function spaces:

$$
\begin{aligned}
\mathcal{C} & :=\left\{c(\mathbf{x}) \in H^{1}(\Omega) \mid c(\mathbf{x})=c^{\mathrm{p}}(\mathbf{x}) \text { on } \partial \Omega\right\} \\
\mathcal{W} & :=\left\{w(\mathbf{x}) \in H^{1}(\Omega) \mid w(\mathbf{x})=0 \text { on } \partial \Omega\right\}
\end{aligned}
$$

where $H^{1}(\Omega)$ is a standard Sobolev space [51]. For weak solutions, the regularity of the diffusivity tensor can be relaxed as follows:

$$
\int_{\Omega} \operatorname{tr}\left[\mathbf{D}(\mathbf{x})^{\mathrm{T}} \mathbf{D}(\mathbf{x})\right] \mathrm{d} \Omega<+\infty
$$


where $\operatorname{tr}[\bullet]$ is the standard trace operator used in tensor algebra and continuum mechanics [56]. Given two fields $a(\mathbf{x})$ and $b(\mathbf{x})$ on a set $\mathcal{D}$, the standard $L_{2}$ inner-product over $\mathcal{D}$ will be denoted as follows:

$$
(a ; b)_{\mathcal{D}}=\int_{\mathcal{D}} a(\mathbf{x}) \cdot b(\mathbf{x}) \mathrm{d} \mathcal{D}
$$

The subscript on the inner-product will be dropped if $\mathcal{D}=\Omega$. The single-field Galerkin formulation for the boundary value problem given by equations $(2.1 \mathrm{a})-(2.1 \mathrm{~b})$ can be written as follows: Find $c(\mathbf{x}) \in \mathcal{C}$, such that we have

$$
\mathcal{B}(w ; c)=L(w) \quad \forall w(\mathbf{x}) \in \mathcal{W}
$$

where the bilinear form and the linear functional are, respectively, defined as follows:

$$
\begin{aligned}
\mathcal{B}(w ; c) & :=(w ; \alpha(\mathbf{x}) c)+(w ; \mathbf{v}(\mathbf{x}) \cdot \operatorname{grad}[c])+(\operatorname{grad}[w] ; \mathbf{D}(\mathbf{x}) \operatorname{grad}[c]) \\
L(w) & :=(w ; f(\mathbf{x}))
\end{aligned}
$$

2.2. Discrete single-field Galerkin formulation. Let the computational domain $\Omega$ be decomposed into "Nele" non-overlapping open sub-domains, which in the finite element context will be elements. That is,

$$
\bar{\Omega}=\bigcup_{e=1}^{\text {Nele }} \bar{\Omega}^{e}
$$

The boundary of $\Omega^{e}$ is denoted as $\partial \Omega^{e}:=\bar{\Omega}^{e}-\Omega^{e}$. Let $\mathbb{P}^{1}\left(\Omega^{e}\right)$ denote the vector space spanned by linear polynomials on the sub-domain $\Omega^{e}$. We shall define the following finite dimensional subsets of $\mathcal{C}$ and $\mathcal{W}$ :

$$
\begin{aligned}
\mathcal{C}^{h} & :=\left\{c^{h}(\mathbf{x}) \in \mathcal{C}\left|c^{h}(\mathbf{x}) \in C^{0}(\bar{\Omega}) ; c^{h}(\mathbf{x})\right|_{\Omega^{e}} \in \mathbb{P}^{1}\left(\Omega^{e}\right) ; e=1, \cdots, \text { Nele }\right\} \\
\mathcal{W}^{h} & :=\left\{w^{h}(\mathbf{x}) \in \mathcal{W}\left|w^{h}(\mathbf{x}) \in C^{0}(\bar{\Omega}) ; w^{h}(\mathbf{x})\right|_{\Omega^{e}} \in \mathbb{P}^{1}\left(\Omega^{e}\right) ; e=1, \cdots, \text { Nele }\right\}
\end{aligned}
$$

A corresponding finite element formulation can be written as follows: Find $c^{h}(\mathbf{x}) \in \mathcal{C}^{h}$, such that we have

$$
\mathcal{B}\left(w^{h} ; c^{h}\right)=L\left(w^{h}\right) \quad \forall w^{h}(\mathbf{x}) \in \mathcal{W}^{h}
$$

where $\mathcal{B}\left(w^{h} ; c^{h}\right)$ and $L\left(w^{h}\right)$ are, respectively, given as follows:

$$
\begin{aligned}
\mathcal{B}\left(w^{h} ; c^{h}\right) & :=\left(w^{h} ; \alpha(\mathbf{x}) c^{h}\right)+\left(w^{h} ; \mathbf{v}(\mathbf{x}) \cdot \operatorname{grad}\left[c^{h}\right]\right)+\left(\operatorname{grad}\left[w^{h}\right] ; \mathbf{D}(\mathbf{x}) \operatorname{grad}\left[c^{h}\right]\right) \\
L\left(w^{h}\right) & :=\left(w^{h} ; f(\mathbf{x})\right)
\end{aligned}
$$

Let " $n_{t}$ " denote the total number of degrees-of-freedom, " $n_{f}$ " denote the free degrees-offreedom, and " $n_{p}$ " be the prescribed degrees-of-freedom for the concentration vector. Obviously, we have $n_{t}=n_{f}+n_{p}$. We assume that $n_{t}, n_{p} \geq 2$. After finite element discretization, the discrete equations for the boundary value problem take the following form:

$$
K c=r
$$

where $\boldsymbol{K} \equiv\left[\boldsymbol{K}_{f f} \mid \boldsymbol{K}_{f p}\right]$ is the stiffness matrix, $\boldsymbol{c} \equiv\left[\boldsymbol{c}_{f}^{\mathrm{T}} \mid \boldsymbol{c}_{p}^{\mathrm{T}}\right]^{\mathrm{T}}$ is the vector containing nodal concentration, and $\boldsymbol{r}=\left[\boldsymbol{r}_{f}\right]^{\mathrm{T}}$ is the corresponding nodal volumetric source vector. The stiffness 
matrices $\boldsymbol{K}, \boldsymbol{K}_{f f}$, and $\boldsymbol{K}_{f p}$ are, respectively, of size $n_{f} \times n_{t}, n_{f} \times n_{f}$, and $n_{f} \times n_{p}$. Correspondingly, the nodal concentration vectors $\boldsymbol{c}, \boldsymbol{c}_{f}$, and $\boldsymbol{c}_{p}$ are of sizes $n_{t} \times 1, n_{f} \times 1$, and $n_{p} \times 1$. Similar inference is applicable to the load vector $\boldsymbol{r}$.

Before we state a discrete version of (weak and strong) maximum and comparison principles, we introduce the required notation. The symbols $\preceq$ and $\succeq$ shall denote component-wise inequalities for vectors and matrices. That is, given two (finite dimensional) vectors $\boldsymbol{a}$ and $\boldsymbol{b}$

$$
\boldsymbol{a} \preceq \boldsymbol{b} \text { means that } a_{i} \leq b_{i} \forall i
$$

Correspondingly, given two matrices $\boldsymbol{A}$ and $\boldsymbol{B}$

$$
\boldsymbol{A} \preceq \boldsymbol{B} \text { means that }(\boldsymbol{A})_{i j} \leq(\boldsymbol{B})_{i j} \forall i, j
$$

Similarly, one can define the symbol $\succeq, \prec$, and $\succ$. In the remainder of this paper, we will be frequently using the symbols $\mathbf{0}$ and $\boldsymbol{O}$, which, respectively, denote a zero vector and a zero matrix.

We shall now briefly outline the main results corresponding to the discrete weak and strong maximum principles in the form of definitions and theorems. Using these results, we shall discuss in detail about discrete comparison principles. However, it should be noted that Theorem 2.8 and its proof are new and have not been discussed elsewhere. We shall also present the necessary and sufficient conditions on the stiffness matrices $\boldsymbol{K}_{f f}$ and $\boldsymbol{K}_{f p}$ to satisfy different versions of discrete maximum principles and the non-negative constraint. For more details, see references $[\mathbf{5 7}, \mathbf{5 8}]$.

Definition 2.5 (Discrete maximum principles [58]). A numerical formulation is said to possess

- the discrete weak maximum principle (DwMP) if

$$
\boldsymbol{r} \preceq \mathbf{0} \quad \text { implies } \quad \max [\boldsymbol{c}] \leq \max \left[0, \max \left[\boldsymbol{c}_{p}\right]\right]
$$

- the discrete strict weak maximum principle (DWMP) if

$$
\boldsymbol{r} \preceq \mathbf{0} \quad \text { implies } \quad \max [\boldsymbol{c}]=\max \left[\boldsymbol{c}_{p}\right]
$$

- the discrete strong maximum principle (DsMP) if it possesses DwMP and satisfies the following condition:

$$
\boldsymbol{r} \preceq \mathbf{0}, \quad \text { and } \quad \max [\boldsymbol{c}]=\max \left[\boldsymbol{c}_{f}\right]=m \geq 0 \quad \text { implies } \quad \boldsymbol{c}=m \mathbf{1}
$$

- the discrete strict strong maximum principle (DSMP) if it possesses DWMP and satisfies the following condition:

$$
\boldsymbol{r} \preceq \mathbf{0}, \quad \text { and } \quad \max [\boldsymbol{c}]=\max \left[\boldsymbol{c}_{f}\right]=m \quad \text { implies } \quad \boldsymbol{c}=m \mathbf{1}
$$

where $\max [\bullet]$ denotes the maximal element of a vector and the symbol $\mathbf{1}$ is the vector whose components are all equal to 1.

THEOREM 2.6 (Necessary and sufficient conditions to satisfy DMPs). The stiffness matrix $\boldsymbol{K}$ given by equation (2.25) is said to possess

- the discrete weak maximum principle $\left(\mathrm{DwMP}_{\boldsymbol{K}}\right)$ if and only if all of the following conditions are satisfied:

$$
\text { (a) } \boldsymbol{K}_{f f}^{-1} \succeq \boldsymbol{O} \quad(\mathrm{b})-\boldsymbol{K}_{f f}^{-1} \boldsymbol{K}_{f p} \succeq \boldsymbol{O} \quad(\mathrm{c})-\boldsymbol{K}_{f f}^{-1} \boldsymbol{K}_{f p} \mathbf{1} \preceq \mathbf{1}
$$


- the discrete strict weak maximum principle $\left(\mathrm{DWMP}_{\boldsymbol{K}}\right)$ if and only if all of the following conditions are satisfied:

$$
\text { (a) } \boldsymbol{K}_{f f}^{-1} \succeq \boldsymbol{O} \quad(\mathrm{b})-\boldsymbol{K}_{f f}^{-1} \boldsymbol{K}_{f p} \succeq \boldsymbol{O} \quad \text { (c) }-\boldsymbol{K}_{f f}^{-1} \boldsymbol{K}_{f p} \mathbf{1}=\mathbf{1}
$$

- the discrete strong maximum principle $\left(\operatorname{DsMP}_{\boldsymbol{K}}\right)$ if and only if all of the following conditions are satisfied:
(a) $\boldsymbol{K}_{f f}^{-1} \succ \boldsymbol{O}$
(b) $-\boldsymbol{K}_{f f}^{-1} \boldsymbol{K}_{f p} \succ \boldsymbol{O}$
(c) $-\boldsymbol{K}_{f f}^{-1} \boldsymbol{K}_{f p} \mathbf{1} \prec \mathbf{1} \quad$ or $\quad-\boldsymbol{K}_{f f}^{-1} \boldsymbol{K}_{f p} \mathbf{1}=\mathbf{1}$

- the discrete strict strong maximum principle $\left(\operatorname{DSMP}_{\boldsymbol{K}}\right)$ if and only if all of the following conditions are satisfied:

$$
\text { (a) } \boldsymbol{K}_{f f}^{-1} \succ \boldsymbol{O} \quad(\mathrm{b})-\boldsymbol{K}_{f f}^{-1} \boldsymbol{K}_{f p} \succ \boldsymbol{O} \quad(\mathrm{c})-\boldsymbol{K}_{f f}^{-1} \boldsymbol{K}_{f p} \mathbf{1}=\mathbf{1}
$$

Proof. For a proof, see reference [58].

DEFINITION 2.7 (Discrete weak and strong comparison principles). A numerical formulation is said to possess

- the discrete weak comparison principle (DwCP) if it satisfies DwMP, and

$$
\boldsymbol{c}_{1} \preceq \boldsymbol{c}_{2} \text { on } \partial \Omega \text { and } \boldsymbol{r}_{1} \preceq \boldsymbol{r}_{2} \text { in } \Omega \quad \text { implies } \quad \boldsymbol{c}_{1} \preceq \boldsymbol{c}_{2} \text { in } \bar{\Omega}
$$

- the discrete strong comparison principle (DsCP) if it satisfies DsMP, and

$$
\boldsymbol{c}_{1} \preceq \boldsymbol{c}_{2} \text { on } \partial \Omega \text { and } \quad \boldsymbol{r}_{1} \prec \boldsymbol{r}_{2} \text { in } \Omega \quad \text { implies } \quad \boldsymbol{c}_{1} \prec \boldsymbol{c}_{2} \text { in } \Omega
$$

THEOREM 2.8 (Necessary and sufficient conditions to satisfy DCPs). Let $\boldsymbol{c}_{1}$ and $\boldsymbol{c}_{2}$ be two nodal concentration vectors corresponding to the volumetric source vectors $\boldsymbol{r}_{1}$ and $\boldsymbol{r}_{2}$ based on the equation (2.25). If $\boldsymbol{c}_{1}$ and $\boldsymbol{c}_{2}$ satisfy DwMP and the hypothesis of DwCP (i.e., $\boldsymbol{c}_{1} \preceq \boldsymbol{c}_{2}$ on $\partial \Omega$ and $\boldsymbol{r}_{1} \preceq \boldsymbol{r}_{2}$ in $\Omega$ ), then a necessary and sufficient condition to satisfy the discrete weak comparison principle $\left(\mathrm{DwCP}_{\boldsymbol{K}}\right.$ ) (which means that $\boldsymbol{c}_{1} \preceq \boldsymbol{c}_{2}$ in $\bar{\Omega}$ ) is that the stiffness matrix $\boldsymbol{K}$ possess $\mathrm{DwMP}_{\boldsymbol{K}}$ (which is given by equation (2.32) in Theorem 2.6).

If $\boldsymbol{c}_{1}$ and $\boldsymbol{c}_{2}$ satisfy DsMP and the hypothesis of $\mathrm{DsCP}$, (i.e., $\boldsymbol{c}_{1} \preceq \boldsymbol{c}_{2}$ on $\partial \Omega$ and $\boldsymbol{r}_{1} \prec \boldsymbol{r}_{2}$ in $\Omega)$, then a necessary and sufficient condition to satisfy the discrete strong comparison principle $\left(\mathrm{DsCP}_{\boldsymbol{K}}\right.$ ) (which means that $\boldsymbol{c}_{1} \prec \boldsymbol{c}_{2}$ in $\Omega$ ) is that the stiffness matrix $\boldsymbol{K}$ possess $\operatorname{DsMP}_{\boldsymbol{K}}$ (which is given by equation (2.34) in Theorem 2.6).

Proof. For convenience, let us define the following:

$$
\begin{aligned}
& \boldsymbol{c}_{3}:=\boldsymbol{c}_{1}-\boldsymbol{c}_{2} \\
& \boldsymbol{r}_{3}:=\boldsymbol{r}_{1}-\boldsymbol{r}_{2}
\end{aligned}
$$

Clearly, $\boldsymbol{c}_{3}$ and $\boldsymbol{r}_{3}$ satisfy the following:

$$
K c_{3}=r_{3}
$$


Necessary condition to satisfy $\operatorname{DwCP}_{\boldsymbol{K}}$ : Let $\boldsymbol{c}_{1} \preceq \boldsymbol{c}_{2}$ in $\bar{\Omega}$, which implies that $\boldsymbol{c}_{3} \preceq \mathbf{0}$ in $\bar{\Omega}$. The hypothesis of $\mathrm{DwCP}_{\boldsymbol{K}}$ and the fact that $\boldsymbol{c}_{3} \preceq \mathbf{0}$ in $\bar{\Omega}$ imply the following:

$$
\begin{aligned}
& \boldsymbol{r}_{3} \preceq \mathbf{0} \quad \text { in } \Omega \\
& \boldsymbol{c}_{3} \preceq \mathbf{0} \text { on } \partial \Omega \\
& \max \left[\boldsymbol{c}_{3}\right] \leq 0 \text { on } \partial \Omega \\
& \max _{\bar{\Omega}}\left[\boldsymbol{c}_{3}\right] \leq \max \left[0, \max _{\partial \Omega}\left[\boldsymbol{c}_{3}\right]\right]=0
\end{aligned}
$$

which implies that $\boldsymbol{c}_{3}$ satisfies DwMP (based on equation (2.28) in Definition 2.5). But vector $\boldsymbol{c}_{3}$ also satisfies equation (2.39). Hence, according to equation (2.32) and the hypothesis of Theorem 2.6, it is evident that $\boldsymbol{K}$ must possess $\operatorname{DwMP}_{\boldsymbol{K}}$. This completes the proof for the necessary condition to satisfy $\mathrm{DwCP}_{\boldsymbol{K}}$.

Sufficient condition to satisfy $\mathrm{DwCP}_{\boldsymbol{K}}$ : It is given that $\boldsymbol{c}_{1}$ and $\boldsymbol{c}_{2}$ satisfy DwMP. Equations $(2.38 \mathrm{a})-(2.38 \mathrm{~b})$ and $\mathrm{DwCP}_{\boldsymbol{K}}$ imply that

$$
\begin{array}{ll}
\boldsymbol{c}_{3} \preceq \mathbf{0} & \text { on } \partial \Omega \\
\boldsymbol{r}_{3} \preceq \mathbf{0} & \text { in } \Omega
\end{array}
$$

If the stiffness matrix $\boldsymbol{K}$ possess $\mathrm{DwMP}_{\boldsymbol{K}}$, it is evident from Theorem 2.6 and equations (2.39), (2.41a)-(2.41b) that vector $\boldsymbol{c}_{3}$ satisfies DwMP. Hence, from Definition 2.5 and equations (2.28), (2.41a)-(2.41b), we have the following result:

$$
\max _{\bar{\Omega}}\left[\boldsymbol{c}_{3}\right] \leq \max \left[0, \max _{\partial \Omega}\left[\boldsymbol{c}_{3}\right]\right]=0
$$

From equation (2.42), it is evident that the least upper bound for any component of vector $\boldsymbol{c}_{3}$ is equal to zero. Hence, we have $\boldsymbol{c}_{3} \preceq \mathbf{0}$ in $\bar{\Omega}$. This implies that $\boldsymbol{c}_{1} \preceq \boldsymbol{c}_{2}$ on $\bar{\Omega}$, which completes the proof for the sufficient condition to satisfy $\mathrm{DwCP}_{\boldsymbol{K}}$.

Necessary condition to satisfy $\mathrm{DsCP}_{K}$ : Following the arguments about the proof for the necessary condition to satisfy the $\mathrm{DwCP}_{\boldsymbol{K}}$ property, it is evident that $\boldsymbol{c}_{3}$ satisfies DwMP. In addition, we are given that $\boldsymbol{c}_{1} \prec \boldsymbol{c}_{2}$ in $\Omega$. This implies $\boldsymbol{c}_{3} \prec \mathbf{0}$ in $\Omega$. Based on the hypothesis of $\mathrm{DsCP}_{\boldsymbol{K}}$ and utilizing the fact that $\boldsymbol{c}_{3} \prec \mathbf{0}$ in $\Omega$ yields the following:

$$
\begin{array}{ll}
\max \left[\boldsymbol{c}_{3}\right] \leq 0 & \text { on } \partial \Omega \\
\max \left[\boldsymbol{c}_{3}\right]<0 & \text { in } \Omega
\end{array}
$$

This means that vector $\mathbf{0}$ is the least upper bound for $\boldsymbol{c}_{3}$ in $\bar{\Omega}$, and any component of $\boldsymbol{c}_{3}$ is strictly less than zero in the interior of the domain $\Omega$. From equation (2.43a) and $(2.43 \mathrm{~b})$, it is clear that the non-negative maximum value for vector $\boldsymbol{c}_{3}$ occurs on the boundary $\partial \Omega$. From equation (2.30) in Definition 2.5, it follows that vector $\boldsymbol{c}_{3}$ satisfies DsMP. Hence, according to conditions specified by equation (2.34) and the hypothesis of Theorem 2.6, it is evident that $\boldsymbol{K}$ must possess the DsMP $\boldsymbol{K}$ property. This completes the proof for the necessary condition to satisfy $\operatorname{DsCP}_{\boldsymbol{K}}$.

Sufficient condition to satisfy $\mathrm{DsCP}_{\boldsymbol{K}}$ : Given that $\boldsymbol{c}_{1}$ and $\boldsymbol{c}_{2}$ satisfy DsMP. Under the assumptions of $\mathrm{DsCP}_{\boldsymbol{K}}$ and from equations (2.38a)-(2.38b), we have the following relations:

$$
\begin{array}{ll}
\boldsymbol{r}_{3} \prec \mathbf{0} & \text { in } \Omega \\
\boldsymbol{c}_{3} \preceq \mathbf{0} & \text { on } \partial \Omega
\end{array}
$$


If the stiffness matrix $\boldsymbol{K}$ possess $\operatorname{DsMP}_{\boldsymbol{K}}$, it is evident from Theorem 2.6 and equations (2.39), (2.44a)-(2.44b) that vector $\boldsymbol{c}_{3}$ satisfies DsMP. Hence, by appealing to Definition 2.5 and equation (2.30), if vector $\boldsymbol{c}_{3}$ does not attain a non-negative maximum value at an interior point of $\Omega$, then we have the following result:

$$
\max _{\Omega}\left[\boldsymbol{c}_{3}\right]<\max \left[0, \max _{\partial \Omega}\left[\boldsymbol{c}_{3}\right]\right]=0
$$

which implies that each component of vector $\boldsymbol{c}_{3}$ is less than zero. Hence, we have $\boldsymbol{c}_{1} \prec \boldsymbol{c}_{2}$ in $\Omega$. Suppose, if vector $\boldsymbol{c}_{3}$ attains a non-negative maximum value at an interior point of $\Omega$, then according to the surmise of DsMP, we first need to satisfy DwMP. So from equation (2.28), we have the following relation:

$$
\max _{\bar{\Omega}}\left[\boldsymbol{c}_{3}\right] \leq \max \left[0, \max _{\partial \Omega}\left[\boldsymbol{c}_{3}\right]\right]=0
$$

Secondly, according to DsMP, we also need to satisfy the equation (2.30). These conditions in terms of vector $\boldsymbol{c}_{3}$ are given as follows:

$$
\begin{aligned}
\max _{\bar{\Omega}}\left[\boldsymbol{c}_{3}\right] & =\max _{\Omega}\left[\boldsymbol{c}_{3}\right]=m \geq 0 \\
\boldsymbol{c}_{3} & =m \mathbf{1} \quad \text { in } \bar{\Omega}
\end{aligned}
$$

From equations (2.46) and $(2.47 \mathrm{a})-(2.47 \mathrm{~b})$, it is evident that $m=0$; which implies that $\boldsymbol{c}_{3}=\mathbf{0}$. Thus, we have $\boldsymbol{c}_{1}=\boldsymbol{c}_{2}$ in $\bar{\Omega}$. But from equation (2.39), it is obvious that $\boldsymbol{r}_{3}=\mathbf{0}$ in $\Omega$, which contradicts the hypothesis of $\operatorname{DsCP}_{\boldsymbol{K}}$ given by the equation (2.44a). Hence, we have the final result $\boldsymbol{c}_{1} \prec \boldsymbol{c}_{2}$ in $\Omega$, which completes the proof for the sufficient condition to satisfy DsCP $\boldsymbol{K}$.

In the next section, we shall discuss the various factors (i.e., mesh restrictions, numerical formulations, and post-processing methods) that influence the satisfaction of discrete versions of maximum principles, comparison principles, and the non-negative constraint.

\section{YET ANOTHER LOOK AT CONTINUOUS AND DISCRETE PRINCIPLES}

Based on the finite element methodology outlined in subsection 2.2, we shall analyze the properties that the stiffness matrix $\boldsymbol{K}$ inherits from the continuous problem. An important attribute that the discrete system needs to have in order to mimic the mathematical properties that the continuous system possesses is that the stiffness matrix $\boldsymbol{K}_{f f}$ has to be a (reducible or irreducible) monotone matrix. The part (a) in all the equations (2.32)-(2.35) of Theorem 2.6 corresponds to reducibility or irreducibility of $\boldsymbol{K}_{f f}$.

On general computational grids, it is well-known that the stiffness matrix $\boldsymbol{K}_{f f}$ obtained via low-order finite element discretization might not be a monotone matrix $[\mathbf{1 2}, \mathbf{3 9}, \mathbf{5 8}]$. So, the discrete single-field Galerkin formulation might (or shall) violate the non-negative constraint, discrete maximum principles, and discrete comparison principles on unstructured computational meshes $[\mathbf{1 2}, \mathbf{4 0}-\mathbf{4 2}, \mathbf{5 7}, \mathbf{5 8}]$. The violation is more severe if the diffusion tensor is anisotropic. One of the ways to overcome such unphysical values for concentration and preserve the discrete properties is to restrict the element shape and size in a computational mesh. This can be achieved by developing sufficient mesh conditions under which $\boldsymbol{K}_{f f}$ is ensured to be a reducibly or irreducibly diagonally dominant matrix [59]. Before we discuss such a class of monotone matrices, which are easily amenable for deriving mesh restrictions, some important remarks on various DMPs and their 
relationship to DCPs and NC are in order. We would like to emphasize that such a comprehensive discussion is not reported elsewhere in the literature.

3.1. Simply connected vs. multiple connected domains. For many applications in mathematics, sciences, and engineering, it is necessary to at least satisfy the weak or strict weak maximum principle. But there are numerous cases where in it is required to satisfy a strong version of the maximum principle $[\mathbf{3 9}, \mathbf{5 7}]$. In such scenarios, geometry and topology of the domain play a vital role. According to the hypothesis of Theorem 2.3, it is evident that a strong maximum principle exists if the domain is simply connected (see reference [52, Chapter 3]). However, one should not immediately conclude that if a domain is not simply connected, then a strong maximum principle will not exist $[\mathbf{5 3}, \mathbf{5 8}]$.

In a discrete setting, Ishihara [57], Drăgănescu et.al. [39], and Mincsovics and Hovárth [58] have conducted various numerical experiments related to discrete strong maximum principles for multiple connected domains. They performed analysis related to satisfaction of $\operatorname{DsMP}_{\boldsymbol{K}}$ and $\mathrm{DSMP}_{\boldsymbol{K}}$ for various non-obtuse and acute triangulations for multiple connected domains. In particular, Mincsovics and Hovárth discuss various interesting examples related to the irreducibility property of the stiffness matrix $\boldsymbol{K}_{f f}$ when the domain is not simply connected. In all of their examples, they solve the following equations:

$$
\begin{array}{ll}
\alpha c-\Delta c=0 & \text { in } \Omega \\
c(\mathbf{x})=c^{\mathrm{p}}(\mathbf{x}) & \text { on } \partial \Omega
\end{array}
$$

where the linear decay $\alpha=0$ or $\alpha=128$. Through numerical experiments, the authors demonstrate that even though the triangulation satisfies the non-obtuse or acute angled mesh condition (proposed by Ciarlet and Raviart [12]), it is not guaranteed to fulfill either DsMP or DSMP. This means that non-obtuse [15] and well-centered triangulation [13] of any given domain will always satisfy the weak DMPs, but need not satisfy the strong DMPs.

Within the context of directed graphs $[\mathbf{3 9}, \mathbf{5 9}]$, there is a one-to-one correspondence between irreducibility of the stiffness matrix $\boldsymbol{K}_{f f}$ and the interior vertices of the computational mesh [30]. In order to satisfy the discrete (strong and strictly strong) maximum principle, the mesh has to be interiorly connected, which in turn implies that $\boldsymbol{K}_{f f}$ has to be irreducible [60]. By interiorly connected mesh, we mean that any pair of interior vertices of the mesh are connected at least by an interior edge path [39]. Hence, $\boldsymbol{K}_{f f}^{-1} \succ \mathbf{0}$ and $-\boldsymbol{K}_{f f}^{-1} \boldsymbol{K}_{f p} \succ \mathbf{0}$ in Theorem 2.6 correspond to this discrete connectedness property of the computational mesh $[\mathbf{3 9}, \mathbf{5 8}]$. However, it should be noted that irreducibility is a necessary condition, but not sufficient. For other details on numerical aspects related to mesh connectivity, see references [58, Section 4, Figures 1-4], [39], and [30].

3.2. Minimum principles, and non-negative and min-max constraints. Due to linearity of the operator $\mathcal{L}$, similar theorems corresponding to minimum principles and the non-negative constraint for equations (2.1a) $-(2.1 \mathrm{~b})$ can be derived. To obtain the non-negative solution and corresponding min-max constraint on $c(\mathbf{x})$, we shall appeal to the continuous weak minimum/minimummaximum principle, which can be written as follows $[\mathbf{5 1}, \mathbf{5 2}]$ :

LEMMA 3.1 (Continuous weak minimum/minimum-maximum principle). Let $\mathcal{L}$ be a uniformly elliptic operator satisfying the conditions given by $(2.9 \mathrm{a})-(2.9 \mathrm{c})$ and $\mathbf{D}(\mathbf{x})$ be continuously differentiable. Given that $\mathcal{L}[c] \geq 0, c^{\mathrm{p}}(\mathbf{x}) \geq 0$, and $c(\mathbf{x}) \in C^{2}(\Omega) \cap C^{0}(\bar{\Omega})$, then $c(\mathbf{x})$ possess a continuous 
weak minimum principle, which is given as follows:

$$
\min _{\mathbf{x} \in \bar{\Omega}}[c(\mathbf{x})] \geq \min \left[0, \min _{\mathbf{x} \in \partial \Omega}[c(\mathbf{x})]\right]
$$

Moreover, if $\mathcal{L}[c]=0$, then we obtain the classical weak minimum-maximum principle for $c(\mathbf{x})$ in $\bar{\Omega}$, which is given as follows:

$$
\min \left[0, \min _{\mathbf{x} \in \partial \Omega}[c(\mathbf{x})]\right] \leq c(\mathbf{x}) \leq \max \left[0, \max _{\mathbf{x} \in \partial \Omega}[c(\mathbf{x})]\right]
$$

Proof. For a proof, see references $[\mathbf{5 1 , 5 2}]$.

It is evident from equation (3.2) that for $f(\mathbf{x}) \geq 0$ and $c^{\mathrm{p}}(\mathbf{x}) \geq 0$, we have $c(\mathbf{x}) \geq 0$ for any $\mathbf{x} \in \bar{\Omega}$. Correspondingly, a discrete version of continuous weak minimum principle and weak minimum-maximum principle is given as follows:

DEFINITION 3.2 (Discrete weak minimum/minimum-maximum principle). A numerical formulation is said to possess

- the discrete weak minimum principle if

$$
\boldsymbol{r} \succeq \mathbf{0} \quad \text { implies } \quad \min [\boldsymbol{c}] \geq \min \left[0, \min \left[\boldsymbol{c}_{p}\right]\right]
$$

- the discrete weak minimum-maximum principle if

$$
\boldsymbol{r}=\mathbf{0} \quad \text { implies } \quad c_{\min } \mathbf{1} \preceq c \preceq c_{\max } \mathbf{1}
$$

where $c_{\min }:=\min \left[\boldsymbol{c}_{p}\right], c_{\max }:=\max \left[\boldsymbol{c}_{p}\right]$, and $\min [\bullet]$ denotes the minimal element of a vector.

3.3. High-order finite element methods. An attribute of low-order finite elements, which plays a central role in designing non-negative formulations for diffusion-type equations, is that the shape functions for these elements are monotonic and do not change their sign within the element [42]. Moreover, they are convenient to generate computational meshes for complex geometries [61], to perform error analysis [62], and for adaptive local mesh refinement [63]. High-order finite elements are widely use for solving smooth problems, as one can obtain exponential convergence under high-order interpolations for these problems. But the shape functions of high-order finite elements change the sign within an element, which makes them not suitable under most of the current non-negative formulations (e.g., see reference $[\mathbf{4 2 , 6 4 ]}$ ). The conditions presented in this paper will also not be applicable to high-order finite elements for the same reason of change in sign of interpolation functions within an element.

As compared to low-order finite element methods, the discrete counterparts of continuous weak and strong maximum principles for high-order finite element methods is not well understood yet. This is because of the complicated task related to the test of non-negativity of a multivariate polynomial [65]. It should be noted that construction of non-negative high-order shape functions for finite element methods is still an unsolved problem and its roots can be traced back to the famous Hilbert's $17^{\text {th }}$ problem $[\mathbf{6 6}, \mathbf{6 7}]$. Within the context of variational methods, probably, the research works by Ciarlet $[\mathbf{6 8}, \mathbf{6 9}]$ are the first attempt to develop high-order non-negative shape functions to satisfy a discrete maximum principle. This study is based on a general theory of discrete Green's function (DGF) for uniformly elliptic linear partial differential operators. Later, various attempts were made by different researchers to develop shape functions and derive mesh restrictions based on the DGF approach. Most of them are pertinent to one-dimensional problems or particular cases of isotropic diffusion. The conditions to be met for high-order elements to satisfy 
DMPs based on DGF methodology are much more stringent and have a less broad scope for general applications. Furthermore, one should be aware that the discrete analogues of continuous Green's functions are applicable only for linear problems, and cannot be extended to to non-linear problems (such as semi-linear and quasi-linear elliptic partial differential equations). Hence, such a method will have limited scope. For more details, one can consult the following references $[\mathbf{6 5}, \mathbf{7 0}, \mathbf{7 1}]$.

3.4. Relationship between various DCPs and DMPs. It is evident from Definitions 2.5, 2.7, and 3.2; Lemma 3.1; and Theorems 2.6 and 2.8 that if a numerical formulation satisfies either $\mathrm{DwCP} / \mathrm{DwCP}_{\boldsymbol{K}}$ or $\mathrm{DsCP} / \mathrm{DsCP}_{\boldsymbol{K}}$, then it automatically obeys $\mathrm{DwMP}_{\mathrm{DwMP}} \boldsymbol{K}$ and $\mathrm{NC}$. Figure 5 illustrates a graphical representation among various numerical solution spaces that satisfy different DMPs and DCPs within the context of mesh restrictions. By a (finite dimensional) numerical solution space $\mathcal{V}_{\mathfrak{p}}$, we mean a set of numerical solutions $\left\{\boldsymbol{c}_{i}\right\}_{i=1}^{n}$, which satisfy a given discrete property given by $\mathfrak{p}$. For example, if a concentration vector $\boldsymbol{c}_{i}$ corresponding to a given volumetric source vector $\boldsymbol{r}_{i}$ satisfies the discrete property DwMP, then $\boldsymbol{c}_{i} \in \mathcal{V}_{\mathrm{DwMP}}$. It should be noted that within the context of DMPs, DCPs, and NC; $\mathcal{V}_{\mathfrak{p}}$ is not a vector space. This is because if $\boldsymbol{c}_{i} \in \mathcal{V}_{\mathfrak{p}}$, then according to non-negative property $-\boldsymbol{c}_{i} \notin \mathcal{V}_{\mathfrak{p}}$, which is one of the properties needed for $\mathcal{V}_{\mathfrak{p}}$ to be a vector space.

Herein, we would like to emphasize that the route taken to satisfy $\mathfrak{p}$ is very important. One can fulfill a discrete property $\mathfrak{p}$ in numerous ways. In general, this is achieved by either placing mesh restrictions or developing a new (non-negative or monotone or monotonicity based) numerical formulation or through various post-processing methods. Couple of these techniques are developed based along the lines similar to Theorem 2.6 and others based on Definition 2.5. But it should be noted that developing numerical formulations accordant to Theorem 2.6 is much more difficult than that of Definition 2.5. This is because in order to satisfy Theorem 2.6, we need to place restrictions on the stiffness matrices $\boldsymbol{K}_{f f}$ and $\boldsymbol{K}_{f p}$. On the other hand, the hypothesis of Definition 2.5 does not assume any particular constraints on $\boldsymbol{K}$. Hence, we would like to differentiate between the set of discrete properties given by $\operatorname{DwMP}_{\boldsymbol{K}}, \mathrm{DWMP}_{\boldsymbol{K}}, \operatorname{DsMP}_{\boldsymbol{K}}, \mathrm{DSMP}_{\boldsymbol{K}}, \mathrm{DwCP}_{\boldsymbol{K}}$, and $\mathrm{DsCP}_{\boldsymbol{K}}$ to that of DwMP, DWMP, DsMP, DSMP, DwCP, and DsCP.

In spite of the fact that there are several numerical methods available to satisfy a given discrete property $\mathfrak{p}$, from the characterization of $\mathcal{V}_{\mathfrak{p}}$, it is evident that the resulting numerical solution spaces will be the same (for example, we have $\mathcal{V}_{\mathrm{DwCP}_{K}} \equiv \mathcal{V}_{\mathrm{DwCP}}$ ). From Theorems 2.6 and 2.8, it is evident that among various DMPs and DCPs, we have the following set inclusions:

$$
\begin{aligned}
& \mathcal{V}_{\mathrm{DSMP}_{K}} \subset \mathcal{V}_{\mathrm{DsMP}_{K}} \subset \mathcal{V}_{\mathrm{DWMP}_{K}} \subset \mathcal{V}_{\mathrm{DwMP}_{K}} \\
& \mathcal{V}_{\mathrm{DsCP}_{K}} \subset \mathcal{V}_{\mathrm{DwCP}_{K}}
\end{aligned}
$$

But it should be noted that a similar type of enclosure for numerical solution spaces between DMPs and DCPs does not hold:

$$
\begin{aligned}
& \mathcal{V}_{\mathrm{DwCP}_{K}} \not \subset \mathcal{V}_{\mathrm{DWMP}_{K}} \\
& \mathcal{V}_{\mathrm{DwCP}_{K}} \not \subset \mathcal{V}_{\mathrm{DsMP}_{K}} \\
& \mathcal{V}_{\mathrm{DwCP}_{K}} \not \subset \mathcal{V}_{\mathrm{DSMP}_{K}} \\
& \mathcal{V}_{\mathrm{DsCP}_{K}} \not \subset \mathcal{V}_{\mathrm{DSMP}_{K}}
\end{aligned}
$$

The reason for such a non-enclosure stems from the hypothesis of Theorems 2.6 and 2.8, wherein we only need to satisfy $\mathrm{DwMP}_{\boldsymbol{K}}$ for $\mathrm{DwCP}_{\boldsymbol{K}}$ and $\operatorname{DsMP}_{\boldsymbol{K}}$ for $\operatorname{DsCP}_{\boldsymbol{K}}$. In a discrete setting, a numerical methodology may inherit one or more than one of these discrete principles and in some 
cases none. Now, we shall discuss in detail a class of numerical formulations, which satisfy a certain discrete property $\mathfrak{p}$. We shall epitomize our findings based on various popular research works in literature, which span across different disciplines, such as computational geometry, optimization theory, numerical linear algebra, and partial differential equations.

(a) Isotropic and anisotropic non-obtuse angle conditions: Recently, Huang and co-workers [28-30] were able to satisfy certain discrete properties through mesh restrictions, which are based on anisotropic $\mathcal{M}$-uniform mesh generation techniques. However, their theoretical investigation is mainly restricted to linear simplicial elements, specifically, the three-node triangular element and four-node tetrahedral element. But one should note that it is difficult to extend the procedure outlined by Huang and co-workers to multi-linear elements, such as the four-node quadrilateral element, six-node wedge element, and eight-node brick element. This is because the partial derivatives of the shape functions for multi-linear finite elements are not constant (for more details, see subsection 4.4 of this paper, which discusses mesh restrictions for a rectangular element). Nevertheless, constructing a WCT mesh [13] or an anisotropic $\mathcal{M}$ uniform triangular mesh $[\mathbf{7 2}, \mathbf{7 3}]$ that satisfies various DMPs for an arbitrary domain is still an open problem [74]. The key-concept we would like to emphasize is that the numerical solutions obtained for isotropic diffusion-type equations using WCT meshes and anisotropic diffusiontype equations using the diffusivity tensor based anisotropic $\mathcal{M}$-uniform meshes satisfy all versions of discrete maximum principles. In addition, if the hypothesis of $\mathrm{DwCP}_{\boldsymbol{K}}$ and $\mathrm{DsCP}_{\boldsymbol{K}}$ is satisfied, then these meshes also satisfy all versions of discrete comparison principles.

(b) Non-linear finite volume and mimetic finite difference methods: Le Potier's method [35] and Lipnikov et al. [75] are some of the noteworthy works in the direction of FVM that satisfy the non-negative constraint, but do not possess a discrete version of the comparison principles and the maximum principles. However, it should be noted that recently these authors have developed techniques based on non-linear finite volume methods $[\mathbf{3 5}, \mathbf{3 7}]$ and mimetic finite difference methods [34] to satisfy various versions of DMPs for a certain specific class of linear self-adjoint elliptic operators. But it should be noted that there is no discussion on satisfying various DCPs.

(c) Optimization-based finite element methods: Based on the works by Liska and Shashkov [40] and Nakshatrala and co-workers $[\mathbf{1 1}, \mathbf{4 1}-\mathbf{4 3}]$, the optimization-based low-order finite element methods, under certain conditions (when $\boldsymbol{K}_{f f}$ is symmetric and positive definite), can be written as follows:

$$
\begin{aligned}
& \boldsymbol{K}_{f f} \boldsymbol{c}_{f}=\boldsymbol{r}-\boldsymbol{K}_{f p} \boldsymbol{c}_{p}+\boldsymbol{\lambda}_{\min }-\boldsymbol{\lambda}_{\max } \\
& c_{\min }^{*} \mathbf{1} \preceq \boldsymbol{c}_{f} \preceq c_{\max }^{*} \mathbf{1} \\
& \boldsymbol{\lambda}_{\min } \succeq \mathbf{0} \\
& \boldsymbol{\lambda}_{\max } \succeq \mathbf{0} \\
& \left(\boldsymbol{c}_{f}-c_{\min }^{*} \mathbf{1}\right) \cdot \boldsymbol{\lambda}_{\min }=0 \\
& \left(c_{\max }^{*} \mathbf{1}-\boldsymbol{c}_{f}\right) \cdot \boldsymbol{\lambda}_{\max }=0
\end{aligned}
$$

where $c_{\min }^{*}$ and $c_{\max }^{*}$ are the minimum and maximum concentration values possible in $\bar{\Omega}$. These values can be obtained based on the boundary conditions and a prior knowledge about the solution. $\boldsymbol{\lambda}_{\min }$ is the vector of Lagrange multipliers corresponding to the constraint $c_{\min }^{*} \mathbf{1} \preceq \boldsymbol{c}_{f}$ 
and similarly $\boldsymbol{\lambda}_{\max }$ is the vector of Lagrange multipliers corresponding to the constraint $\boldsymbol{c}_{f} \preceq$ $c_{\max }^{*} 1$.

Based on the nature of constraints, one can satisfy different discrete principles and it should be emphasized that DsMP, DSMP, DwCP, and DsCP can be fulfilled only under certain conditions. If either $\boldsymbol{r} \succ \mathbf{0}$ or $\boldsymbol{r} \prec \mathbf{0}$, then the non-negative constraint and the weaker versions of discrete minimum/maximum principles can be satisfied by specifying either $c_{\min }^{*}$ or $c_{\max }^{*}$. But in the case of $\boldsymbol{r}=\mathbf{0}$, both $c_{\min }^{*}$ and $c_{\max }^{*}$ can be prescribed based on the Dirichlet boundary conditions; moreover, according to Definition 3.2 and from equation (3.5), we have $c_{\min }^{*}=c_{\min }$ and $c_{\max }^{*}=c_{\max }$. However, one should note that if the qualitative and quantitative nature of the solution is known a prior, then one can satisfy all of the discrete versions of (weak and strong) maximum principles by specifying $c_{\min }^{*}$ and $c_{\max }^{*}$ in the Karush-Kuhn-Tucker conditions given by equations (3.8a)-(3.8f). In general, these methods do not inherit a discrete strong maximum principle and a discrete comparison principle. For more details, a counter example is shown in the reference [11, Section 4, Figure 1]). Nevertheless, satisfying DsMP, DSMP, DwCP, and $\mathrm{DsCP}$ is still an open problem and are interesting topics to investigate in future endeavors.

(d) Variationally inconsistent methods: In literature, there are various post-processing methods [46-48] available that can recover certain discrete properties if a prior information about the numerical solution is known. However, one should note that such methods are variationally inconsistent. A summary of the above discussion between various discrete principles within the context of FDS, MFDM, FVM, FEM and PP based methods is pictorially described in Figure 6.

In the next section, we shall derive sufficient conditions on the three-node triangular element and four-node quadrilateral element to satisfy discrete versions of comparison principles, maximum principles, and the non-negative constraint.

\section{MESH RESTRICTIONS TO SATISFY DISCRETE PRINCIPLES}

In this section, we shall utilize and build upon the research works of Huang and co-workers [28-30, 72] for linear second-order elliptic equations. We first present, without proofs, relevant mathematical and geometrical results required to obtain mesh restrictions for simplicial elements. We will then use these results to construct mesh restriction theorems and generate various types of triangulations (see Algorithm 1 and the discussion in subsection 4.3) using the open source mesh generators, such as Gmsh [76] and BAMG [19] available in the FreeFem ++ software package [20,21]. It should be emphasized that these results cannot be extended to Q4 element, as this element is not simplicial. For more details on mesh restrictions for Q4 element, see subsection 4.4 of this paper.

Let $\hat{\boldsymbol{x}}_{1}, \hat{\boldsymbol{x}}_{2}, \ldots, \hat{\boldsymbol{x}}_{n d+1}$ denote the vertices of an arbitrary simplex $\Omega_{e} \in \mathcal{T}_{h}$, where $\mathcal{T}_{h}$ is a simplicial triangulation of the domain $\Omega$. The subscript ' $h$ ' in the triangulation $\mathcal{T}_{h}$ corresponds to the maximum element size (which will be described later in this section, see equation (4.20a)). Based on various values of $h$, we have an affine family of such simplicial meshes denoted by $\left\{\mathcal{T}_{h}\right\}$. Designate the total number of vertices and the corresponding interior vertices of $\mathcal{T}_{h}$ by ' $N v$ ' and ' $N i v$ '. The edge matrix of $\Omega_{e}$, which is denoted by $\boldsymbol{E}_{\Omega_{e}}$, is defined as follows:

$$
\boldsymbol{E}_{\Omega_{e}}:=\left[\hat{\boldsymbol{x}}_{2}-\hat{\boldsymbol{x}}_{1}, \hat{\boldsymbol{x}}_{3}-\hat{\boldsymbol{x}}_{1}, \ldots, \hat{\boldsymbol{x}}_{n d+1}-\hat{\boldsymbol{x}}_{1}\right] \quad \forall \Omega_{e} \in \mathcal{T}_{h}
$$

then an edge connecting vertices $\hat{\boldsymbol{x}}_{p}$ and $\hat{\boldsymbol{x}}_{q}$ of $\Omega_{e}$ is denoted as $e_{p q}$. Correspondingly, the edge vector $\boldsymbol{e}_{p q, \Omega_{e}}$ (which can be expressed as a linear combination of the elements corresponding to the 
edge matrix $\left.\boldsymbol{E}_{\Omega_{e}}\right)$ and the element boundary $\partial \Omega_{e}$ in-terms of $\hat{\boldsymbol{x}}_{p}, \hat{\boldsymbol{x}}_{q}$, and $e_{p q}$ are given by:

$$
\begin{aligned}
\boldsymbol{e}_{p q, \Omega_{e}} & =\hat{\boldsymbol{x}}_{q}-\hat{\boldsymbol{x}}_{p} \quad \forall p, q=1,2, \ldots, n d+1 \text { and } p \neq q \\
\partial \Omega_{e} & =\bigcup_{\substack{p, q=1 \\
p \neq q}}^{n d+1} e_{p q}
\end{aligned}
$$

Following references $[\mathbf{2 8}, \mathbf{7 7}]$, a set of $\boldsymbol{q}$-vectors corresponding to this edge matrix $\boldsymbol{E}_{\Omega_{e}}$ are defined as follows:

$$
\begin{aligned}
& \boldsymbol{E}_{\Omega_{e}}^{-\mathrm{T}}:=\left[\boldsymbol{q}_{2}, \boldsymbol{q}_{3}, \ldots, \boldsymbol{q}_{n d+1}\right] \\
& \boldsymbol{q}_{1}+\sum_{p=2}^{n d+1} \boldsymbol{q}_{p}=\mathbf{0} \quad \forall \Omega_{e} \in \mathcal{T}_{h}
\end{aligned}
$$

Let $\varphi_{p_{g}}$ denote the linear basis function associated with the $p_{g^{-}}$-th global vertex in the triangulation $\mathcal{T}_{h}$. Then, $\left\{\varphi_{p_{g}}\right\}_{p_{g}=1}^{N v}$ and $\left\{\varphi_{p_{g}}\right\}_{p_{g}=1}^{N i v}$ span the respective finite dimensional subsets $\mathcal{C}^{h}$ and $\mathcal{W}^{h}$ given by equations $(2.22 \mathrm{a})-(2.22 \mathrm{~b})$. Denote the face opposite to vertex $\hat{\boldsymbol{x}}_{p}$ by $F_{p}$ and the corresponding unit inward normal pointing towards the vertex $\hat{\boldsymbol{x}}_{p}$ by $\mathbf{n}_{p}$. The perpendicular distance (or the height) from vertex $\hat{\boldsymbol{x}}_{p}$ to face $F_{p}$ is denoted by $h_{p}$. In the case of $2 \mathrm{D}$, the above set of geometrical properties are pictorially described in Figure 7.

Definition 4.1 (Positive linear maps). Let $\mathbb{M}_{n}:=\mathbb{M}_{n \times n}(\mathbb{R})$ be the set of all real matrices of size $n \times n$, which forms a vector space over the field $\mathbb{R}$. A linear map $\Phi: \mathbb{M}_{n} \rightarrow \mathbb{M}_{k}$ is called positive if $\Phi(\boldsymbol{A})$ is positive semi-definite whenever $\boldsymbol{A}$ is positive semi-definite. Similarly, $\Phi$ is called strictly positive if $\Phi(\boldsymbol{A})$ is positive definite whenever $\boldsymbol{A}$ is positive definite.

THEOREM 4.2 (Strictly positive linear mapping of anisotropic diffusivity). Let $\Phi[\bullet]:=$ $\int_{\Omega_{e}}[\bullet] \mathrm{d} \Omega$. Show that $\Phi[\bullet]$ is a linear map and $\Phi[\mathbf{D}(\mathbf{x})]$ is symmetric, uniformly elliptic, and bounded above.

Proof. From Definition 4.1, it is evident that $\Phi[\bullet]$ is a linear map and $\Phi[\mathbf{D}(\mathbf{x})]$ is symmetric. From equations (2.6) and (2.21), we have $\boldsymbol{\xi} \cdot \mathbf{D}(\mathbf{x}) \boldsymbol{\xi}>0$ and meas $\left(\Omega_{e}\right)>0$. It is well known that Lebesgue integration of a scalar for a strictly positive measure is always greater than zero. Hence, $\int_{\Omega_{e}} \boldsymbol{\xi} \cdot \mathbf{D}(\mathbf{x}) \boldsymbol{\xi} \mathrm{d} \Omega>0 \quad \forall \mathbf{x} \in \Omega_{e}$. Now, integrating equation (2.6) over $\Omega_{e}$ results in the following relation:

$$
0<\gamma_{\min } \boldsymbol{\xi} \cdot \boldsymbol{\xi} \leq \frac{1}{\operatorname{meas}\left(\Omega_{e}\right)} \int_{\Omega_{e}} \boldsymbol{\xi} \cdot \mathbf{D}(\mathbf{x}) \boldsymbol{\xi} \mathrm{d} \Omega \leq \gamma_{\max } \boldsymbol{\xi} \cdot \boldsymbol{\xi} \quad \forall \boldsymbol{\xi} \in \mathbb{R}^{n d} \backslash\{\mathbf{0}\} \text { and } \forall \mathbf{x} \in \Omega_{e}
$$

where the positive constants $\gamma_{\min }$ and $\gamma_{\max }$ are respectively the integral average of minimum and maximum eigenvalues of $\mathbf{D}(\mathbf{x})$ over $\Omega_{e}$. These are given as follows:

$$
\begin{aligned}
\gamma_{\min } & :=\frac{1}{\operatorname{meas}\left(\Omega_{e}\right)} \int_{\Omega_{e}} \lambda_{\min }(\mathbf{x}) \mathrm{d} \Omega \\
\gamma_{\max } & :=\frac{1}{\operatorname{meas}\left(\Omega_{e}\right)} \int_{\Omega_{e}} \lambda_{\max }(\mathbf{x}) \mathrm{d} \Omega
\end{aligned}
$$


Since the vector $\boldsymbol{\xi}$ is independent of $\mathbf{x}$ and $\Omega_{e}$, we can interchange the order of integration. This gives us the following equation:

$$
0<\gamma_{\min } \boldsymbol{\xi} \cdot \boldsymbol{\xi} \leq \frac{1}{\operatorname{meas}\left(\Omega_{e}\right)} \boldsymbol{\xi} \cdot \Phi[\mathbf{D}(\mathbf{x})] \boldsymbol{\xi} \leq \gamma_{\max } \boldsymbol{\xi} \cdot \boldsymbol{\xi} \quad \forall \boldsymbol{\xi} \in \mathbb{R}^{n d} \backslash\{\mathbf{0}\} \text { and } \forall \mathbf{x} \in \Omega_{e}
$$

which shows that $\Phi[\mathbf{D}(\mathbf{x})]$ is a strictly positive linear map of $\mathbf{D}(\mathbf{x})$ and indeed preserves its properties.

4.1. Geometrical properties and finite element analysis of simplicial elements. Based on the above notation, we have the following important mathematical results relating the linear basis functions, finite element matrices, and geometrical properties of simplicial elements.

(1) The integral element average anisotropic diffusivity $\widetilde{\boldsymbol{D}}_{\Omega_{e}}$ is given by:

$$
\widetilde{\boldsymbol{D}}_{\Omega_{e}}:=\frac{1}{\operatorname{meas}\left(\Omega_{e}\right)} \int_{\Omega_{e}} \mathbf{D}(\mathbf{x}) \mathrm{d} \Omega \quad \forall \Omega_{e} \in \mathcal{T}_{h}
$$

is a strictly positive linear map (see Definition 4.1 and Theorem 4.2).

(2) For any arbitrary simplicial element $\Omega_{e} \in \mathcal{T}_{h}$, the vector $\boldsymbol{q}_{p}$ associated with the face $F_{p}$, the gradient of the linear basis function $\operatorname{grad}\left[\varphi_{p_{g}}\right]$, the unit inward normal $\mathbf{n}_{p}$, and the height $h_{p}$ are related as follows $[\mathbf{7 4}, \mathbf{7 7}]$ :

$$
\boldsymbol{q}_{p}=\left.\operatorname{grad}\left[\varphi_{p_{g}}\right]\right|_{\Omega_{e}}=\frac{\mathbf{n}_{p}}{h_{p}} \quad \forall p=1,2, \ldots, n d+1
$$

(3) The dihedral angle $\beta_{p q}$ (measured in the Euclidean metric) between any two faces $F_{p}$ and $F_{q}$ is related to $\boldsymbol{q}$-vectors $\left(\mathbf{q}_{p}\right.$ and $\left.\mathbf{q}_{q}\right)$ and unit inward normals $\left(\mathbf{n}_{p}\right.$ and $\left.\mathbf{n}_{q}\right)$ by the following equation $[\mathbf{2 8}, \mathbf{2 9}]$ :

$$
\cos \left(\beta_{p q}\right)=-\mathbf{n}_{p} \cdot \mathbf{n}_{q}=-\frac{\mathbf{q}_{p} \cdot \mathbf{q}_{q}}{\left\|\mathbf{q}_{p}\right\|\left\|\mathbf{q}_{q}\right\|} \quad \forall p, q=1,2, \ldots, n d+1 \quad \text { and } \quad p \neq q
$$

where $\|\bullet\|$ is the standard Euclidean norm. Similarly, the dihedral angle $\beta_{p q, \widetilde{\boldsymbol{D}}_{\Omega_{e}}^{-1}}$ measured in $\widetilde{\boldsymbol{D}}_{\Omega_{e}}^{-1}$ metric is given as follows:

$$
\cos \left(\beta_{p q, \widetilde{\boldsymbol{D}}_{\Omega_{e}}^{-1}}\right)=-\frac{\mathbf{q}_{p} \cdot \widetilde{\boldsymbol{D}}_{\Omega_{e}} \mathbf{q}_{q}}{\left\|\mathbf{q}_{p}\right\|_{\widetilde{\boldsymbol{D}}_{\Omega_{e}}}\left\|\mathbf{q}_{q}\right\|_{\widetilde{\boldsymbol{D}}_{\Omega_{e}}}} \quad \forall p, q=1,2, \ldots, n d+1 \quad \text { and } \quad p \neq q
$$

where $\|\bullet\|_{\widetilde{\boldsymbol{D}}_{\Omega_{e}}}$ denotes the norm in $\widetilde{\boldsymbol{D}}_{\Omega_{e}}$ metric. For example, $\left\|\mathbf{q}_{p}\right\|_{\widetilde{\boldsymbol{D}}_{\Omega_{e}}}=\sqrt{\mathbf{q}_{p} \cdot \widetilde{\boldsymbol{D}}_{\Omega_{e}} \mathbf{q}_{p}}$.

(4) For any simplex $\Omega_{e} \in \mathcal{T}_{h}$, the gradient of the linear basis functions $\left(\operatorname{grad}\left[\varphi_{p_{g}}\right]\right.$ and $\left.\operatorname{grad}\left[\varphi_{q_{g}}\right]\right)$, the dihedral angle $\beta_{p q}$, and heights $\left(h_{p}\right.$ and $\left.h_{q}\right)$ are related as follows $[\mathbf{2 8}, \mathbf{2 9}]$ :

$$
\begin{aligned}
\operatorname{meas}\left(\Omega_{e}\right)\left(\left.\left.\operatorname{grad}\left[\varphi_{p_{g}}\right]\right|_{\Omega_{e}} \cdot \operatorname{grad}\left[\varphi_{q_{g}}\right]\right|_{\Omega_{e}}\right)= & -\frac{\operatorname{meas}\left(\Omega_{e}\right) \cos \left(\beta_{p q}\right)}{h_{p} h_{q}} \\
& \forall p, q=1,2, \ldots, n d+1 \quad \text { and } \quad p \neq q
\end{aligned}
$$

and in $2 \mathrm{D}$, equation (4.11) reduces to:

$$
\begin{aligned}
\operatorname{meas}\left(\Omega_{e}\right)\left(\left.\left.\operatorname{grad}\left[\varphi_{p_{g}}\right]\right|_{\Omega_{e}} \cdot \operatorname{grad}\left[\varphi_{q_{g}}\right]\right|_{\Omega_{e}}\right)= & -\frac{\cot \left(\beta_{p q}\right)}{2} \\
& \forall p, q=1,2, \ldots, n d+1 \quad \text { and } \quad p \neq q
\end{aligned}
$$


(5) For any arbitrary simplicial element $\Omega_{e} \in \mathcal{T}_{h}$, the integral element average anisotropic diffusivity $\widetilde{\boldsymbol{D}}_{\Omega_{e}}$, the gradient of the linear basis functions $\left(\operatorname{grad}\left[\varphi_{p_{g}}\right]\right.$ and $\left.\operatorname{grad}\left[\varphi_{q_{g}}\right]\right)$, the dihedral angle $\beta_{p q, \widetilde{\boldsymbol{D}}_{\Omega_{e}}^{-1}}$ measured in $\widetilde{\boldsymbol{D}}_{\Omega_{e}}^{-1}$ metric, and heights $\left(h_{p}\right.$ and $\left.h_{q}\right)$ are related as follows $[\mathbf{2 9}]$ :

$$
\begin{aligned}
\operatorname{meas}\left(\Omega_{e}\right)\left(\left.\left.\operatorname{grad}\left[\varphi_{p_{g}}\right]\right|_{\Omega_{e}} \cdot \widetilde{\boldsymbol{D}}_{\Omega_{e}} \operatorname{grad}\left[\varphi_{q_{g}}\right]\right|_{\Omega_{e}}\right)= & -\frac{\operatorname{meas}\left(\Omega_{e}\right) \cos \left(\beta_{p q, \widetilde{\boldsymbol{D}}_{\Omega_{e}}^{-1}}\right.}{\left\|\mathbf{q}_{p}\right\|_{\widetilde{\boldsymbol{D}}_{\Omega_{e}}^{-1}}^{-1}\left\|\mathbf{q}_{q}\right\|_{\widetilde{\boldsymbol{D}}_{\Omega_{e}}}^{-1}} \\
& \forall p, q=1,2, \ldots, n d+1 \text { and } p \neq q
\end{aligned}
$$

and in $2 \mathrm{D}$, equation $(4.13)$ reduces to:

$$
\begin{aligned}
\operatorname{meas}\left(\Omega_{e}\right)\left(\left.\left.\operatorname{grad}\left[\varphi_{p_{g}}\right]\right|_{\Omega_{e}} \cdot \widetilde{\boldsymbol{D}}_{\Omega_{e}} \operatorname{grad}\left[\varphi_{q_{g}}\right]\right|_{\Omega_{e}}\right)= & -\frac{\operatorname{det}\left[\widetilde{\boldsymbol{D}}_{\Omega_{e}}\right]^{\frac{1}{2}}}{2} \cot \left(\beta_{p q, \widetilde{\boldsymbol{D}}_{\Omega_{e}}^{-1}}\right) \\
& \forall p, q=1,2, \ldots, n d+1 \text { and } p \neq q
\end{aligned}
$$

4.2. Sufficient conditions for a three-node triangular element. Using the mathematical results outlined in subsection 4.1, we shall present various sufficient conditions on the T3 element to satisfy different types of discrete properties. In general, there are two different approaches to obtain sufficient conditions. The first approach, which shall be called global stiffness restriction method, involves manipulating the entries of the global stiffness matrix, so that it is either weakly or strictly diagonally dominant (see Theorems 4.3 and 4.4) based on the nature of $\alpha(\mathbf{x})$. This means that $\boldsymbol{K}_{f f}^{-1}$ exists and $\boldsymbol{K}_{f f}^{-1} \succeq \mathbf{0}$. The component wise entries of $\boldsymbol{K}_{f f}$ satisfy the following conditions:

(a) Positive diagonal entries: $\left(\boldsymbol{K}_{f f}\right)_{i i}>0$,

(b) Non-positive off-diagonal entries: $\left(\boldsymbol{K}_{f f}\right)_{i j} \leq 0 \quad \forall i \neq j$, and

one of the following two conditions:

(c) Strict diagonal dominance of rows: $\left|\left(\boldsymbol{K}_{f f}\right)_{i i}\right|>\sum_{i \neq j}\left|\left(\boldsymbol{K}_{f f}\right)_{i j}\right| \quad \forall i, j$

(c) Weak diagonal dominance of rows: $\left|\left(\boldsymbol{K}_{f f}\right)_{i i}\right| \geq \sum_{i \neq j}\left|\left(\boldsymbol{K}_{f f}\right)_{i j}\right| \quad \forall i, j$

The second method, which shall be called local stiffness restriction method, engineers on stiffness matrices at the local level, so that they are weakly diagonally dominant. Once we ascertain that all of the local stiffness matrices are weakly diagonally dominant, then the standard finite element assembly process [78] guarantees that the global stiffness matrix $\boldsymbol{K}_{f f}$ is monotone and weakly diagonally dominant. In particular, if $\alpha(\mathbf{x})>0$, then $\boldsymbol{K}_{f f}$ is strictly diagonally dominant. It should be noted that the above three conditions are sufficient, but not necessary, for the global stiffness matrix $\boldsymbol{K}_{f f}$ to be monotone.

4.2.1. Global and local stiffness restriction methods. In general, to get an explicit analytical formula for $\boldsymbol{K}_{f f}^{-1}$ is extremely difficult and not practically viable. Hence, it is not feasible to find mesh restrictions based on the condition that $\boldsymbol{K}_{f f}^{-1} \succeq \mathbf{0}$. So an expedient route to obtain monotone stiffness matrices through mesh restrictions is by means of weakly or strictly diagonally dominant matrices, which form a subset to the class of monotone matrices [60, Section 3, Corollary 3.20 and Corollary 3.21]. The obvious edge being that there is no need to compute $\left(\boldsymbol{K}_{f f}^{-1}\right)_{i j}$ explicitly. Based on the global stiffness restriction method, we shall now present stronger and weaker mesh restriction theorems. A brief and concise proof to the theorems shall be put forth, which is along 
similar lines to the one presented in references $[\mathbf{2 9}, \mathbf{3 0}]$. These mesh restriction theorems shall be used in constructing triangular meshes to satisfy different discrete principles (see subsection 4.3).

THEOREM 4.3 (Anisotropic non-obtuse angle condition). If any nd-simplicial mesh satisfies the following anisotropic non-obtuse angle condition:

$$
\begin{gathered}
0<\frac{h_{p}\|\boldsymbol{v}\|_{\infty, \bar{\Omega}_{e}}}{(n d+1) \Lambda_{m i n, \widetilde{\boldsymbol{D}}_{\Omega_{e}}}}+\frac{h_{p} h_{q}\|\alpha\|_{\infty, \bar{\Omega}_{e}}}{(n d+1)(n d+2) \Lambda_{m i n, \widetilde{\boldsymbol{D}}_{\Omega_{e}}}} \leq \cos \left(\beta_{p q, \widetilde{\boldsymbol{D}}_{\Omega_{e}}^{-1}}\right) \\
\forall p, q=1,2, \cdots, n d+1, p \neq q, \Omega_{e} \in \mathcal{T}_{h}
\end{gathered}
$$

then we have the following three results:

- The global stiffness matrix $\boldsymbol{K}_{f f}$ is (reducibly/irreducibly) weakly diagonally dominant if $\alpha(\mathbf{x}) \geq 0$ and is (reducibly/irreducibly) strictly diagonally dominant if $\alpha(\mathbf{x})>0$ for all $\mathrm{x} \in \bar{\Omega}$.

- The discrete single-field Galerkin formulation given by equations (2.24a)-(2.24b) in combination with equations (4.7)-(4.14) satisfies $\operatorname{DwMP}_{\boldsymbol{K}} / \mathrm{DWMP}_{\boldsymbol{K}}$, where $\|\boldsymbol{v}\|_{\infty, \bar{\Omega}_{e}}$ and $\|\alpha\|_{\infty, \bar{\Omega}_{e}}$ are defined as:

$$
\begin{gathered}
\|\boldsymbol{v}\|_{\infty, \bar{\Omega}_{e}}:=\underset{\boldsymbol{x} \in \bar{\Omega}_{e}}{\operatorname{maximize}}\|\boldsymbol{v}(\boldsymbol{x})\| \\
\|\alpha\|_{\infty, \bar{\Omega}_{e}}:=\underset{\boldsymbol{x} \in \bar{\Omega}_{e}}{\operatorname{maximize}} \alpha(\boldsymbol{x})
\end{gathered}
$$

and $\Lambda_{\text {min, } \widetilde{\boldsymbol{D}}_{\Omega_{e}}}$ denotes the minimum eigenvalue of $\widetilde{\boldsymbol{D}}_{\Omega_{e}} ; h_{p}, h_{q}$, and $\beta_{p q, \widetilde{\boldsymbol{D}}_{\Omega_{e}}^{-1}}$ are respectively the heights and metric based dihedral angle opposite to the face $F_{r}$ of element $\Omega_{e}$.

- Moreover, if the triangulation $\mathcal{T}_{h}$ is interiorly connected, then the global stiffness matrix $\boldsymbol{K}_{f f}$ is irreducibly weakly or strictly diagonally dominant based on the nature of $\alpha(\mathbf{x})$ and the discrete single-field Galerkin formulation given by equations (2.24a)-(2.24b) satisfies $\operatorname{DsMP}_{\boldsymbol{K}} / \mathrm{DSMP}_{\boldsymbol{K}}$.

Proof. For proof, see References $[\mathbf{2 9}, \mathbf{3 0}]$.

TheOREM 4.4 (Generalized Delaunay-type angle condition). In 2D, if a simplicial mesh satisfies the following generalized Delaunay-type angle condition (which is much weaker than that of equation (4.15)):

$$
\begin{aligned}
& 0<\frac{1}{2}\left[\beta_{p q, \widetilde{\boldsymbol{D}}_{\Omega_{e}}^{-1}}+\beta_{p q, \widetilde{\boldsymbol{D}}_{\Omega_{e}^{\prime}}^{-1}}\right] \\
& +\frac{1}{2} \operatorname{arccot}\left(\sqrt{\frac{\operatorname{det}\left[\widetilde{\boldsymbol{D}}_{\Omega_{e}^{\prime}}\right]}{\operatorname{det}\left[\widetilde{\boldsymbol{D}}_{\Omega_{e}}\right]}} \cot \left(\beta_{p q, \widetilde{\boldsymbol{D}}_{\Omega_{e}^{\prime}}^{-1}}\right)-\frac{2 \mathfrak{C}_{q, \Omega_{e}, \Omega_{e}^{\prime}}}{\sqrt{\operatorname{det}\left[\widetilde{\boldsymbol{D}}_{\Omega_{e}}\right]}}\right) \\
& +\frac{1}{2} \operatorname{arccot}\left(\sqrt{\frac{\operatorname{det}\left[\widetilde{\boldsymbol{D}}_{\Omega_{e}}\right]}{\operatorname{det}\left[\widetilde{\boldsymbol{D}}_{\Omega_{e}^{\prime}}\right]}} \cot \left(\beta_{p q, \widetilde{\boldsymbol{D}}_{\Omega_{e}}^{-1}}\right)-\frac{2 \mathfrak{C}_{q, \Omega_{e}, \Omega_{e}^{\prime}}}{\sqrt{\operatorname{det}\left[\widetilde{\boldsymbol{D}}_{\Omega_{e}^{\prime}}\right]}}\right) \leq \pi
\end{aligned}
$$

for every internal edge $e_{p q}$ connecting the $p$-th and $q$-th vertices of the elements $\Omega_{e}$ and $\Omega_{e}^{\prime}$ that share this common edge (see Figure 7), then we have the following three results:

- The global stiffness matrix $\boldsymbol{K}_{f f}$ is (reducibly/irreducibly) weakly diagonally dominant if $\alpha(\mathbf{x}) \geq 0$ and is (reducibly/irreducibly) strictly diagonally dominant if $\alpha(\mathbf{x})>0$ for all $\mathrm{x} \in \bar{\Omega}$. 
- The discrete single-field Galerkin formulation given by equations (2.24a)-(2.24b) in association with equations (4.7)-(4.14) satisfies $\mathrm{DwMP}_{\boldsymbol{K}} / \mathrm{DWMP}_{\boldsymbol{K}}$. The quantities $h_{p, \Omega_{e}}$ and $h_{q, \Omega_{e}}$ are the heights of $\Omega_{e}, h_{p, \Omega_{e}^{\prime}}$ and $h_{q, \Omega_{e}^{\prime}}$ are the heights of $\Omega_{e}^{\prime}, \beta_{p q, \widetilde{\boldsymbol{D}}_{\Omega_{e}}^{-1}}$ and $\beta_{p q, \widetilde{\boldsymbol{D}}_{\Omega_{e}^{\prime}}^{-1}}$ are the relevant metric based dihedral angles in the elements $\Omega_{e}$ and $\Omega_{e}^{\prime}$ that face the edge $e_{p q}$, and the parameters $\|\boldsymbol{v}\|_{\infty, \bar{\Omega}_{e}}$ and $\|\alpha\|_{\infty, \bar{\Omega}_{e}}$ are evaluated in $\Omega_{e}$ based on the equations (4.16a)-(4.16b). Similarly, $\|\boldsymbol{v}\|_{\infty, \bar{\Omega}_{e}^{\prime}}$ and $\|\alpha\|_{\infty, \bar{\Omega}_{e}^{\prime}}$ are evaluated in $\Omega_{e}^{\prime}$. The constant $\mathfrak{C}_{q, \Omega_{e}, \Omega_{e}^{\prime}}$ in equation (4.17) is given as follows:

$$
\mathfrak{C}_{q, \Omega_{e}, \Omega_{e}^{\prime}}:=\operatorname{meas}\left(\Omega_{e}\right)\left(\frac{\|\boldsymbol{v}\|_{\infty, \bar{\Omega}_{e}}}{3 h_{q, \Omega_{e}}}+\frac{\|\alpha\|_{\infty, \bar{\Omega}_{e}}}{12}\right)+\operatorname{meas}\left(\Omega_{e}^{\prime}\right)\left(\frac{\|\boldsymbol{v}\|_{\infty, \bar{\Omega}_{e}^{\prime}}}{3 h_{q, \Omega_{e}^{\prime}}}+\frac{\|\alpha\|_{\infty, \bar{\Omega}_{e}^{\prime}}}{12}\right)
$$

- Additionally, if the triangulation $\mathcal{T}_{h}$ is interiorly connected, then the global stiffness matrix $\boldsymbol{K}_{f f}$ is irreducibly weakly or strictly diagonally dominant based on the nature of $\alpha(\mathbf{x})$ and the discrete single-field Galerkin formulation given by equations (2.24a)-(2.24b) satisfies $\operatorname{DsMP}_{\boldsymbol{K}} / \mathrm{DSMP}_{\boldsymbol{K}}$.

Proof. For proof, see References $[\mathbf{2 9}, \mathbf{3 0}]$.

Each method (global stiffness restriction method or local stiffness restriction method) has it own advantages and disadvantages. The advantage of the global stiffness restriction method is that we can operate at a global level. This gives us different types of relationships between various mesh parameters, which can be used in generating different types of triangulations, such as DelaunayVoronoi, non-obtuse, well-centered, and anisotropic $\mathcal{M}$-uniform finite element meshes. For instance, Taylor series expansion of equations (4.15) and (4.17) gives the following restrictions on the metric based dihedral angles for all simplicial elements in a triangulation:

$$
\begin{aligned}
& 0<\beta_{p q, \widetilde{\boldsymbol{D}}_{\Omega_{e}}^{-1}} \leq \frac{\pi}{2}-\mathcal{O}\left(h\|\boldsymbol{v}\|_{\infty, \mathcal{T}_{h}}+h^{2}\|\alpha\|_{\infty, \mathcal{T}_{h}}\right) \\
& 0<\frac{1}{2}\left[\beta_{p q, \widetilde{\boldsymbol{D}}_{\Omega_{e}}^{-1}}+\beta_{p q, \widetilde{\boldsymbol{D}}_{\Omega_{e}^{\prime}}^{-1}}\right]+\frac{1}{2} \operatorname{arccot}\left(\sqrt{\frac{\operatorname{det}\left[\widetilde{\boldsymbol{D}}_{\Omega_{e}^{\prime}}\right]}{\operatorname{det}\left[\widetilde{\boldsymbol{D}}_{\Omega_{e}}\right]}} \cot \left(\beta_{p q, \widetilde{\boldsymbol{D}}_{\Omega_{e}^{\prime}}^{-1}}\right)\right) \\
& +\frac{1}{2} \operatorname{arccot}\left(\sqrt{\frac{\operatorname{det}\left[\widetilde{\boldsymbol{D}}_{\Omega_{e}}\right]}{\operatorname{det}\left[\widetilde{\boldsymbol{D}}_{\Omega_{e}^{\prime}}\right]}} \cot \left(\beta_{p q, \widetilde{\boldsymbol{D}}_{\Omega_{e}}^{-1}}\right)\right) \leq \pi-\mathcal{O}\left(h\|\boldsymbol{v}\|_{\infty, \mathcal{T}_{h}}+h^{2}\|\alpha\|_{\infty, \mathcal{T}_{h}}\right)
\end{aligned}
$$

where the maximum element size $h$, maximum element normed velocity $\|\boldsymbol{v}\|_{\infty, \mathcal{T}_{h}}$, and maximum element normed linear reaction coefficient $\|\alpha\|_{\infty, \mathcal{T}_{h}}$ are given as follows:

$$
\begin{aligned}
h & :=\max _{\Omega_{e} \in \mathcal{T}_{h}}\left[h_{\max , \Omega_{e}}\right] \\
\|\boldsymbol{v}\|_{\infty, \mathcal{T}_{h}} & :=\max _{\Omega_{e} \in \mathcal{T}_{h}}\left[\|\boldsymbol{v}\|_{\infty, \bar{\Omega}_{e}}\right] \\
\|\alpha\|_{\infty, \mathcal{T}_{h}} & :=\max _{\Omega_{e} \in \mathcal{T}_{h}}\left[\|\alpha\|_{\infty, \bar{\Omega}_{e}}\right]
\end{aligned}
$$

where $h_{\max , \Omega_{e}}$ is the maximum possible height in a given simplicial element $\Omega_{e}$, and $\mathcal{O}(\bullet)$ is the standard "big-oh" notation [79]. Specifically, on a $h$-refined triangular mesh, which conforms to Theorems 4.3 and 4.4, then equation (4.19a) implies that all the dihedral angles when measured in 
the metric of $\widetilde{\boldsymbol{D}}_{\Omega_{e}}^{-1}$ have to be $\mathcal{O}\left(h\|\boldsymbol{v}\|_{\infty, \mathcal{T}_{h}}+h^{2}\|\alpha\|_{\infty, \mathcal{T}_{h}}\right)$ acute/non-obtuse and equation (4.19b) indicates that the triangulation needs to be $\mathcal{O}\left(h\|\boldsymbol{v}\|_{\infty, \mathcal{T}_{h}}+h^{2}\|\alpha\|_{\infty, \mathcal{T}_{h}}\right)$ Delaunay.

One downside of the global stiffness restriction approach is that obtaining mesh conditions is mathematically cumbersome. Moreover, extending it to non-simplicial low-order finite elements is extremely hard and is not straightforward. This is because the basis functions $\varphi_{p_{g}}$ spanning the finite dimensional subsets $\mathcal{C}^{h}$ and $\mathcal{W}^{h}$ are multi-linear, which makes $\operatorname{grad}\left[\varphi_{p_{g}}\right]$ on any arbitrary element $\Omega_{e}$ to be non-constant. So most of properties given by equations (4.7)-(4.14) are not valid for low-order finite elements such as the Q4 element and its corresponding elements in higher dimensions.

Now we shall describe the local stiffness restriction method and highlight the pros and cons of using this methodology. For the sake of illustration, we shall consider a pure anisotropic diffusion equation and assume that $\hat{\boldsymbol{x}}_{p}=(0,0), \hat{\boldsymbol{x}}_{q}=(1,0)$, and $\hat{\boldsymbol{x}}_{r}=(a, b)$. Our objective is to find the coordinates $(a, b)$, such that the local stiffness matrix is weakly diagonally dominant for any given type of diffusivity tensor. The local stiffness matrix for an anisotropic diffusion equation based on discrete single-field Galerkin formulation is given by:

$$
\boldsymbol{K}_{e}=\int_{\Omega_{e}} \boldsymbol{B} \boldsymbol{D}(\boldsymbol{x}) \boldsymbol{B}^{\mathrm{t}} \mathrm{d} \Omega
$$

where the matrix $\boldsymbol{B}$ in terms of the coordinates $(a, b)$ is given as follows:

$$
\boldsymbol{B}=\frac{1}{b}\left(\begin{array}{cc}
-b & (a-1) \\
b & -a \\
0 & 1
\end{array}\right)
$$

Since the entries in the matrix $\boldsymbol{B}$ are constants, we have the local stiffness matrix $\boldsymbol{K}_{e}$ given as follows:

$$
\boldsymbol{K}_{e}=\boldsymbol{B}\left(\int_{\Omega_{e}} \boldsymbol{D}(\boldsymbol{x}) \mathrm{d} \Omega\right) \boldsymbol{B}^{\mathrm{t}}
$$

In the subsequent subsections, we present various sufficient conditions through which we can find these coordinates $(a, b)$ and glean information on the possible angles and corresponding shape and size of the triangle PQR.

4.2.2. T3 element for heterogeneous isotropic diffusivity. In this subsection, we consider the case where the diffusivity is isotropic and heterogeneous in the total domain. For this case, we show that the diffusivity does not have any influence on determining the coordinates $(a, b)$. This means that the restrictions we obtain on the coordinates and the angles of the triangle PQR is independent of how the diffusivity is varying across the domain. The following is the local stiffness matrix for scalar heterogeneous isotropic diffusion:

$$
\boldsymbol{K}_{e}=\frac{1}{b^{2}} \int_{\Omega_{e}} D(\boldsymbol{x}) \mathrm{d} \Omega\left(\begin{array}{ccc}
b^{2}+(a-1)^{2} & a-a^{2}-b^{2} & (a-1) \\
a-a^{2}-b^{2} & a^{2}+b^{2} & -a \\
(a-1) & -a & 1
\end{array}\right)
$$


where the integral of the diffusivity $D(\boldsymbol{x})$ over the actual T3 element $\Omega_{e}$ (triangle PQR) is given as follows:

$$
\widetilde{D}=\frac{1}{\operatorname{meas}\left(\Omega_{e}\right)} \int_{\Omega_{e}} D(\boldsymbol{x}) \mathrm{d} \Omega
$$

where meas $\left(\Omega_{e}\right)=\frac{b}{2}$ is the area of the T3 element PQR, which is always positive. The simplified expression for the local stiffness matrix is given as follows:

$$
\boldsymbol{K}_{e}=\frac{\widetilde{D}}{2 b}\left(\begin{array}{ccc}
b^{2}+(a-1)^{2} & a-a^{2}-b^{2} & (a-1) \\
a-a^{2}-b^{2} & a^{2}+b^{2} & -a \\
(a-1) & -a & 1
\end{array}\right)
$$

We shall now present the sufficient conditions so that the matrix $\boldsymbol{K}_{e}$ is weakly diagonally dominant:

Condition No-1. Positive diagonal entries: $\left(\boldsymbol{K}_{e}\right)_{i i}>0 \quad \forall i=1,2,3$. This restriction gives us the following inequalities:

$$
\begin{aligned}
& \left(\boldsymbol{K}_{e}\right)_{11}=\frac{\widetilde{D}}{2 b}\left(b^{2}+(a-1)^{2}\right)>0 \\
& \left(\boldsymbol{K}_{e}\right)_{22}=\frac{\widetilde{D}}{2 b}\left(a^{2}+b^{2}\right)>0 \\
& \left(\boldsymbol{K}_{e}\right)_{33}=\frac{\widetilde{D}}{2 b}>0
\end{aligned}
$$

As $\widetilde{D}>0$ and $b>0$, it is evident that all of the inequalities given by equations $(4.27 \mathrm{a})-(4.27 \mathrm{c})$ are trivially satisfied. Hence, this condition has no effect on obtaining restrictions on coordinates $(a, b)$.

Condition No-2. Weak diagonal dominance of rows: $\left|\left(\boldsymbol{K}_{e}\right)_{i i}\right| \geq \sum_{i \neq j}\left|\left(\boldsymbol{K}_{e}\right)_{i j}\right| \quad \forall i, j$, where $i=1,2,3$ and $j=1,2,3$. This restriction gives the following inequalities:

$$
\begin{aligned}
\left(b^{2}+(a-1)^{2}\right) & \geq\left(a^{2}+b^{2}-a\right)+(1-a) \\
\left(a^{2}+b^{2}\right) & \geq a+\left(a^{2}+b^{2}-a\right) \\
1 & \geq(1-a)+a
\end{aligned}
$$

Note that these inequalities (4.28a)-(4.28c) are trivially satisfied. Hence, this condition has no influence on obtaining restrictions on triangle PQR.

Condition No-3. Non-positive off-diagonal entries: $\left(\boldsymbol{K}_{e}\right)_{i j} \leq 0 \quad \forall i \neq j$, where $i=1,2,3$ and $j=1,2,3$. This restriction gives the following inequalities:

$$
\left(\boldsymbol{K}_{e}\right)_{i j}=\left\{\begin{array}{ll}
\left(\boldsymbol{K}_{e}\right)_{12}=\frac{\widetilde{D}}{2 b}\left(a-a^{2}-b^{2}\right) \leq 0 \\
\left(\boldsymbol{K}_{e}\right)_{13}=\frac{\widetilde{D}}{2 b}(a-1) \leq 0 \\
\left(\boldsymbol{K}_{e}\right)_{23}=\frac{\widetilde{D}}{2 b}(-a) \leq 0 \\
25
\end{array} \quad \forall i \neq j\right.
$$


Using the fact that $\widetilde{D}>0$ and $b>0$, and rearranging the above relations, we get the following inequalities:

$$
\begin{aligned}
\left(a-\frac{1}{2}\right)^{2}+b^{2} & \geq\left(\frac{1}{2}\right)^{2} \\
a & \leq 1 \\
a & \geq 0
\end{aligned}
$$

The region in which the coordinates $(a, b)$ satisfy the above inequalities given by the equations $(4.29 \mathrm{a})-(4.29 \mathrm{c})$ is shown in Figure 8. According to these inequalities (4.29a)-(4.29c), heterogeneity of the scalar diffusivity has no role in obtaining the feasible region for the coordinates $(a, b)$. It is evident from Figure 8 that the interior angles of the triangle PQR are either acute or at most rightangle. Based on the sufficient conditions, one can also notice that an obtuse-angled triangle is not possible. So in order to satisfy discrete comparison principles, discrete maximum principles, and non-negative constraint, the triangulation of a given computational domain must contain acuteangled triangles or right-angled triangles. These three sufficient conditions show that non-obtuse or well-centered triangulations inherit all the three discrete versions of continuous properties of scalar heterogeneous isotropic diffusion equations.

4.2.3. T3 element for heterogeneous anisotropic diffusivity. In this subsection, we consider the case where the diffusivity $\mathbf{D}(\mathbf{x})=\left(\begin{array}{cc}D_{x x}(\mathbf{x}) & D_{x y}(\mathbf{x}) \\ D_{x y}(\mathbf{x}) & D_{y y}(\mathbf{x})\end{array}\right)$ is anisotropic and heterogeneous across the domain. For the sake of brevity and ease of manipulations, we shall drop the symbol ( $\mathbf{x})$ in the components of the diffusivity tensor. Note that the symbol ' $\mathbf{x}$ ' in the components of $\mathbf{D}(\mathbf{x})$ is dropped for the sake of convenience and should not be interpreted as though the diffusivity tensor is constant. As discussed in Section 2, the diffusivity tensor needs to satisfy certain properties. Based on equations (2.2) and (2.6), we derive various results related to $\mathbf{D}(\mathbf{x})$ that will be used in deriving mesh restrictions.

REMARK 4.5. In 2D, it is trivial to show that, if the matrix $\boldsymbol{D}(\boldsymbol{x})$ is symmetric, uniformly elliptic, and bounded above, then its components satisfy the following relations:

$$
\begin{aligned}
& D_{x x}>0 \\
& D_{y y}>0 \\
& D_{x x} D_{y y}>D_{x y}^{2}
\end{aligned}
$$

Let us denote $\epsilon:=\frac{\widetilde{D}_{y y}}{\widetilde{D}_{x x}}$ and $\eta:=\frac{\widetilde{D}_{x y}}{\widetilde{D}_{x x}}$, where $\widetilde{D}_{x x}, \widetilde{D}_{x y}$, and $\widetilde{D}_{y y}$ are the components of matrix $\widetilde{\boldsymbol{D}}_{\Omega_{e}}$ given by equation (4.7). From Theorem 4.2 , it is evident that $\widetilde{D}_{x x}>0, \widetilde{D}_{y y}>0$, and $\widetilde{D}_{x x} \widetilde{D}_{y y}>\widetilde{D}_{x y}^{2}$. So from equation (4.30c), we have $\eta \in(-\sqrt{\epsilon}, \sqrt{\epsilon})$. These two non-dimensional quantities $\epsilon$ and $\eta$ govern the mesh restrictions that we impose on the coordinates $(a, b)$. From equations (4.21) and (4.22), the stiffness matrix for any given anisotropic diffusivity tensor is given as follows:

$$
\boldsymbol{K}_{e}=\left(\begin{array}{ccc}
\frac{\widetilde{D}_{x x} b^{2}-2 \widetilde{D}_{x y} b(a-1)+\widetilde{D}_{y y}(a-1)^{2}}{2 b} & -\frac{\widetilde{D}_{x x} b^{2}+\widetilde{D}_{x y}(b-2 a b)+\widetilde{D}_{y y} a(a-1)}{2 b} & \frac{-\widetilde{D}_{x y} b+\widetilde{D}_{y y}(a-1)}{2 b \widetilde{D}_{x x} b^{2}+\widetilde{D}_{x y}(b-2 a b)+\widetilde{D}_{y y} a(a-1)} \\
\frac{-\widetilde{D}_{x y} b+\widetilde{D}_{y y}(a-1)}{2 b} & \frac{\widetilde{D}_{x x} b^{2}-2 \widetilde{D}_{x y} a b+\widetilde{D}_{y y} a^{2}}{2 b} & \frac{\widetilde{D}_{x y} b-\widetilde{D}_{y y} a}{2 b} \\
\frac{\widetilde{D}_{x y} b-\widetilde{D}_{y y} a}{2 b} & \frac{\widetilde{D}_{y y}}{2 b}
\end{array}\right)
$$

We now present sufficient conditions so that the matrix $\boldsymbol{K}_{e}$ is weakly diagonally dominant. 
Condition No-4. Positive diagonal entries: $\left(\boldsymbol{K}_{e}\right)_{i i}>0 \quad \forall i=1,2,3$, gives the following relations:

$$
\left(\boldsymbol{K}_{e}\right)_{i i}=\left\{\begin{array}{l}
\left(\boldsymbol{K}_{e}\right)_{11}=\frac{\widetilde{D}_{x x} b^{2}-2 \widetilde{D}_{x y} b(a-1)+\widetilde{D}_{y y}(a-1)^{2}}{2 b}>0 \\
\left(\boldsymbol{K}_{e}\right)_{22}=\frac{\widetilde{D}_{x x} b^{2}-2 \widetilde{D}_{x y} a b+\widetilde{D}_{y y} a^{2}}{2 b}>0 \\
\left(\boldsymbol{K}_{e}\right)_{33}=\frac{\widetilde{D}_{y y}}{2 b}>0
\end{array}\right.
$$

As $\widetilde{D}_{y y}>0$ and $b>0$, rearranging the above relations, we have the following restrictions:

$$
\begin{aligned}
& \left(\boldsymbol{K}_{e}\right)_{11}=\left(b \sqrt{\widetilde{D}_{x x}}-|a-1| \sqrt{\widetilde{D}_{y y}}\right)^{2}+2 b|a-1|\left(\sqrt{\widetilde{D}_{x x}} \sqrt{\widetilde{D}_{y y}}-\operatorname{Sgn}[|a-1|] \widetilde{D}_{x y}\right)>0 \\
& \left(\boldsymbol{K}_{e}\right)_{22}=\left(b \sqrt{\widetilde{D}_{x x}}-|a| \sqrt{\widetilde{D}_{y y}}\right)^{2}+2 b|a|\left(\sqrt{\widetilde{D}_{x x}} \sqrt{\widetilde{D}_{y y}}-\operatorname{Sgn}[|a|] \widetilde{D}_{x y}\right)>0 \\
& \left(\boldsymbol{K}_{e}\right)_{33}=\frac{\widetilde{D}_{y y}}{2 b}>0
\end{aligned}
$$

where $\operatorname{Sgn}[\bullet]$ is the standard lignum function (which provides the sign of the real number). It is evident that $\sqrt{\widetilde{D}_{x x}} \sqrt{\widetilde{D}_{y y}}>\widetilde{D}_{x y}$. Hence, equations $(4.32 \mathrm{a})-(4.32 \mathrm{c})$ are trivially satisfied for any abscissa $a$.

Condition No-5. Non-positive off-diagonal entries: $\left(\boldsymbol{K}_{e}\right)_{i j} \leq 0 \quad \forall i \neq j$, where $i=1,2,3$, and $j=1,2,3$. This restriction gives the following relations:

$$
\left(\boldsymbol{K}_{e}\right)_{i j}=\left\{\begin{array}{l}
\left(\boldsymbol{K}_{e}\right)_{12}=-\frac{\widetilde{D}_{x x} b^{2}+\widetilde{D}_{x y}(b-2 a b)+\widetilde{D}_{y y} a(a-1)}{2 b} \leq 0 \\
\left(\boldsymbol{K}_{e}\right)_{13}=\frac{-\widetilde{D}_{x y} b+\widetilde{D}_{y y}(a-1)}{2 b} \leq 0 \\
\left(\boldsymbol{K}_{e}\right)_{23}=\frac{\widetilde{D}_{x y} b-\widetilde{D}_{y y} a}{2 b} \leq 0
\end{array}\right.
$$

Using the parameters $\epsilon, \eta$, and the fact that ordinate $b>0$, we have the following inequalities:

$$
\begin{aligned}
\left(a-\frac{1}{2}\right)^{2}+\left(\frac{b}{\sqrt{\epsilon}}\right)^{2}-2 b\left(\frac{\eta}{\epsilon}\right)\left(a-\frac{1}{2}\right) & \geq\left(\frac{1}{2}\right)^{2} \\
\frac{a-1}{b} & \leq \frac{\eta}{\epsilon} \\
\frac{a}{b} & \geq \frac{\eta}{\epsilon}
\end{aligned}
$$

which dictate the feasible region for coordinates $(a, b)$. For a given $\epsilon$ and by varying $\eta$, which lies between $-\sqrt{\epsilon}$ and $\sqrt{\epsilon}$, we get different feasible regions for $(a, b)$. Herein, we have chosen $\epsilon=10$ and $\eta \in\{-1,0,1\}$. For these values, we have plotted the feasible region based on the inequalities (4.33a)-(4.33c). From Figures 9-14, the following can be inferred based on the feasible region:

- If $\eta=0$, the possible T3 elements are either acute-angled or right-angled triangles.

- If either $\eta<0$ or $\eta>0$, then obtuse-angled triangles are also possible. Moreover, the resulting triangles can be skinny or skewed. 
Condition No-6. Weak diagonal dominance of rows: $\left|\left(\boldsymbol{K}_{e}\right)_{i i}\right| \geq \sum_{i \neq j}\left|\left(\boldsymbol{K}_{e}\right)_{i j}\right| \quad \forall i, j$, where $i=1,2,3$, and $j=1,2,3$. This gives the following relations:

$$
\begin{aligned}
\left(b^{2}-2 \eta b(a-1)+\epsilon(a-1)^{2}\right) & \geq\left(b^{2}+\eta(b-2 a b)+\epsilon a(a-1)\right)+(\eta b-\epsilon(a-1)) \\
\left(b^{2}-2 \eta a b+\epsilon a^{2}\right) & \geq\left(b^{2}+\eta(b-2 a b)+\epsilon a(a-1)\right)(\epsilon a-\eta b) \\
\epsilon & \geq(\eta b-\epsilon(a-1))+(\epsilon a-\eta b)
\end{aligned}
$$

if Condition- 4 and Condition-5 are satisfied, then this condition is trivially satisfied. In a similar fashion, for a general case, where $\hat{\boldsymbol{x}}_{p}=\left(x_{1}, y_{1}\right), \hat{\boldsymbol{x}}_{q}=\left(x_{2}, y_{2}\right)$, and $\hat{\boldsymbol{x}}_{r}=\left(x_{3}, y_{3}\right)$, we have the following conditions based on the local stiffness restriction method:

$$
\begin{array}{r}
\left(y_{1}-y_{3}\right)\left(y_{3}-y_{2}\right)-\eta\left(x_{1}-x_{3}\right)\left(y_{3}-y_{2}\right)-\eta\left(x_{3}-x_{2}\right)\left(y_{1}-y_{3}\right)+\epsilon\left(x_{1}-x_{3}\right)\left(x_{3}-x_{2}\right) \leq 0 \\
\left(y_{2}-y_{1}\right)\left(y_{3}-y_{2}\right)-\eta\left(x_{3}-x_{2}\right)\left(y_{2}-y_{1}\right)-\eta\left(x_{2}-x_{1}\right)\left(y_{3}-y_{2}\right)+\epsilon\left(x_{2}-x_{1}\right)\left(x_{3}-x_{2}\right) \leq 0 \\
\left(y_{1}-y_{3}\right)\left(y_{2}-y_{1}\right)-\eta\left(x_{1}-x_{3}\right)\left(y_{2}-y_{1}\right)-\eta\left(x_{2}-x_{1}\right)\left(y_{1}-y_{3}\right)+\epsilon\left(x_{1}-x_{3}\right)\left(x_{2}-x_{1}\right) \leq 0 \\
\left(x_{1}-x_{3}\right)\left(y_{2}-y_{1}\right)-\left(x_{1}-x_{2}\right)\left(y_{3}-y_{1}\right)>0
\end{array}
$$

where the first three inequalities given by equations $(4.35 \mathrm{a})-(4.35 \mathrm{c})$ are obtained based on the condition that $\left(\boldsymbol{K}_{e}\right)_{i j} \leq 0$. The last inequality given by equation (4.35d) is the result of the condition that meas $\left(\Omega_{e}\right)>0$.

The benefit (attractive feature) of the local stiffness restriction method is that the local stiffness matrix for the discrete Galerkin formulation given by equations (2.24a)-(2.24b) can be calculated quite easily and could be extended to even non-simplicial elements (see subsection 4.4). Using this approach, we can obtain general restrictions and analytical expressions relating various coordinates of an arbitrary mesh element, using popular symbolic packages like Mathematica [80]. But a flip-side of this procedure is that incorporating the inequalities given by equations (4.35a)-(4.35d) in a mesh generator is very difficult and needs further detailed investigation. Additionally, the conditions obtained using this method are stringent and similar to that of Theorem 4.3 given by the global stiffness restriction method (which will be evident based on the numerical examples discussed in the following subsection). Finally, it should be noted that extending the local stiffness restriction method to include advection and linear reaction is straightforward and shall not be dealt with to save space. We shall now present various numerical examples and respective triangular meshes corresponding to different types of $\mathbf{D}(\mathbf{x})$. Using these meshes, we shall analyze and study in detail, which kind of DMPs and DCPs are preserved.

4.3. Numerical examples based on different types of triangulations. In this subsection, we shall first briefly discuss on a metric tensor $\mathcal{M}(\boldsymbol{x})$ to satisfy DCPs, DMPs, and NC. Based on this metric tensor, we shall describe an algorithm to generate various types of DMP-based triangulations (mainly utilizing open source mesh generators, such as Gmsh and BAMG). Simplicial meshes constructed based on $\mathcal{M}(\boldsymbol{x})$ (where the metric tensor $\mathcal{M}(\boldsymbol{x})$ is not equal to a scalar multiple of identity tensor) are called anisotropic $\mathcal{M}$-uniform simplicial meshes. They are uniform in the metric specified by $\mathcal{M}(\boldsymbol{x})[\mathbf{6 1}, \mathbf{7 2}]$ and are of primal importance in satisfying various important discrete properties in the areas of transport of chemical species, fluid mechanics, and porous media applications $[\mathbf{8 1 - 8 3}$. Given a $\mathcal{M}(\boldsymbol{x})$, there are different approaches to generate anisotropic $\mathcal{M}$-uniform simplicial meshes. Some of the notable research works in this direction include blue refinement [84], bubble packing [85], Delaunay-type triangulation [81], directional refinement [86], 
front advancing [85], local refinement and modification [87], and variational mesh generation [88]. In general, the metric tensor $\mathcal{M}(\boldsymbol{x})$ is symmetric and positive definite, and gives relevant information on the shape, size, and orientation of mesh elements in the computational domain.

Let $\chi$ be an affine mapping from the reference element $\Omega_{\text {ref }}$ to background mesh element $\Omega_{e}$. Denote its Jacobian by $\boldsymbol{J}_{\Omega_{e}}$. The affine mapping $\chi$ and its Jacobian $\boldsymbol{J}_{\Omega_{e}}$ are given as follows:

$$
\begin{aligned}
\boldsymbol{x} & =\chi(\hat{\boldsymbol{X}}, \boldsymbol{N})=\hat{\boldsymbol{X}}^{\mathrm{T}} \boldsymbol{N}^{\mathrm{T}} \\
\boldsymbol{J}_{\Omega_{e}} & =\hat{\boldsymbol{X}}^{\mathrm{T}} \boldsymbol{D} \boldsymbol{N}
\end{aligned}
$$

where $\hat{\boldsymbol{X}}$ is the nodal matrix, which comprises of nodal vertices $\left(\hat{\boldsymbol{x}}_{1}, \hat{\boldsymbol{x}}_{2}, \ldots, \hat{\boldsymbol{x}}_{n d+1}\right)$ of an arbitrary simplex $\Omega_{e}$. The elements of vector $N$ consists of shape functions corresponding to $\Omega_{\text {ref }}$. The entries of matrix $\boldsymbol{D} \boldsymbol{N}$ (which are constants for simplicial elements) correspond to the derivatives of shape functions. Straightforward manipulations on equations (4.8) and (4.36b) give the following result:

$$
\boldsymbol{q}_{p, \text { ref }}=\boldsymbol{J}_{\Omega_{e}} \boldsymbol{q}_{p}
$$

where $\boldsymbol{q}_{p \text {,ref }}$ is the corresponding $\boldsymbol{q}$-vector of the reference element $\Omega_{\text {ref }}$. Huang [72] has shown that an anisotropic $\mathcal{M}$-uniform simplicial mesh satisfies the following two conditions:

$$
\begin{aligned}
\rho_{\Omega_{e}} \operatorname{meas}\left(\Omega_{e}\right) & =\frac{\sigma_{h}}{\text { Nele }} \quad \forall \Omega_{e} \in \mathcal{T}_{h} \\
\frac{1}{n d} \operatorname{tr}\left[\boldsymbol{J}_{\Omega_{e}}^{\mathrm{T}} \mathcal{M}_{\Omega_{e}} \boldsymbol{J}_{\Omega_{e}}\right] & =\operatorname{det}\left[\boldsymbol{J}_{\Omega_{e}}^{\mathrm{T}} \mathcal{M}_{\Omega_{e}} \boldsymbol{J}_{\Omega_{e}}\right]^{\frac{1}{n d}} \quad \forall \Omega_{e} \in \mathcal{T}_{h}
\end{aligned}
$$

The quantities $\mathcal{M}_{\Omega_{e}}, \rho_{\Omega_{e}}$, and $\sigma_{h}$ are given as follows:

$$
\begin{aligned}
\mathcal{M}_{\Omega_{e}} & :=\frac{1}{\operatorname{meas}\left(\Omega_{e}\right)} \int_{\Omega_{e}} \mathcal{M}(\boldsymbol{x}) \mathrm{d} \Omega \quad \forall \Omega_{e} \in \mathcal{T}_{h} \\
\rho_{\Omega_{e}} & :=\sqrt{\operatorname{det}\left[\mathcal{M}_{\Omega_{e}}\right]} \forall \Omega_{e} \in \mathcal{T}_{h} \\
\sigma_{h} & :=\sum_{\Omega_{e} \in \mathcal{T}_{h}} \rho_{\Omega_{e}} \operatorname{meas}\left(\Omega_{e}\right)
\end{aligned}
$$

The condition given by the equation (4.38a) is called equidistribution condition and that by equation (4.38b) is called alignment condition. Equidistribution condition decides the size of $\Omega_{e}$, while alignment condition characterizes the shape and orientation of $\Omega_{e}$. From AM-GM inequality [89], equation (4.38b) implies the following:

$$
\boldsymbol{J}_{\Omega_{e}}^{\mathrm{T}} \mathcal{M}_{\Omega_{e}} \boldsymbol{J}_{\Omega_{e}}=\left(\frac{\sigma_{h}}{\text { Nele }}\right)^{\frac{2}{n d}} \boldsymbol{I} \quad \forall \Omega_{e} \in \mathcal{T}_{h}
$$

Now, through trivial manipulations on equations (4.37), (4.10), and (4.40), the metric tensor $\mathcal{M}_{\Omega_{e}}$ has to satisfy the following equation in order to meet various DMPs:

$$
\mathcal{M}_{\Omega_{e}}=\Theta_{\Omega_{e}} \widetilde{\boldsymbol{D}}_{\Omega_{e}}^{-1}
$$

where $\Theta_{\Omega_{e}}$ is an arbitrary piecewise positive scalar constant, which in general is a user-define parameters. Loosely speaking, an anisotropic $\mathcal{M}$-uniform simplicial mesh satisfying (4.41) satisfies weak DMPs. Furthermore, if the resulting simplicial mesh is interiorly connected, then it satisfies strong DMPs. 
For a given type of metric tensor (for example, $\mathcal{M}(\boldsymbol{x})$ given by equation (4.41)), open source mesh generators (such as BAMG, BL2D, and Mmg3d) take this as an input and operate on a background mesh to produce an anisotropic $\mathcal{M}$-uniform simplicial mesh. Algorithm 1 provides a methodology to develop an anisotropic $\mathcal{M}$-uniform simplicial mesh based on a background mesh, and Figure 10 highlights the salient aspects of this algorithm. Nevertheless, it should be noted that Algorithm 1 is a general algorithm to generate DMP-based triangulations (is not limited to either Gmsh or BAMG) for any type of mesh generator, which operates on a background mesh. On the other hand, there are certain open source and commercially available software packages, such as CGAL [90] and Simmetrix [91], which create an anisotropic $\mathcal{M}$-uniform simplicial mesh directly based on the metric tensor $[\mathbf{9 2}, \mathbf{9 3}]$ without the need of background meshes. Investigation of such mesh generators is beyond the scope of this research paper and is neither critical nor central to the ideas discussed here. Before we discuss various numerical examples based on different types of triangulations, we shall now present certain important (mesh-based) non-dimensional numbers relevant to our numerical study. Such a discussion is first of its kind and is not discussed elsewhere.

REMARK 4.6. It should be noted that STEP-7 in Algorithm 1 might be computationally intensive, as we need to solve a series of small constrained optimization problem for each $\Omega_{e, i} \in \widetilde{\mathcal{T}}_{h, i}$ ' and at every iteration level ' $i$ '. The corresponding constrained optimization problems are given as follows:

$$
\begin{aligned}
\|\boldsymbol{v}\|_{e, i}:=\underset{\boldsymbol{x} \in \bar{\Omega}_{e, i}}{\operatorname{maximize}}\|\boldsymbol{v}(\boldsymbol{x})\| & \forall 1 \leq i \leq \text { MaxIters } \\
\|\alpha\|_{e, i}:=\operatorname{maximize}_{\boldsymbol{x} \in \bar{\Omega}_{e, i}} \alpha(\boldsymbol{x}) & \forall 1 \leq i \leq \text { MaxIters }
\end{aligned}
$$

As $\bar{\Omega}_{e, i}$ is convex, triangular, and closed, by half-space representation theorem for convex polytopes [95, Section-2.2.4] equation (4.42a) can be written as follows:

$$
\begin{aligned}
\underset{\boldsymbol{x} \in \mathbb{R}^{2}}{\operatorname{maximize}} & \|\boldsymbol{v}(\boldsymbol{x})\| \\
\text { subject to } & \boldsymbol{A}_{e, i} \boldsymbol{x} \preceq \boldsymbol{b}_{e, i} \quad \forall 1 \leq i \leq \text { MaxIters }
\end{aligned}
$$

where $\boldsymbol{A}_{e, i}$ is a $3 \times 2$ matrix and $\boldsymbol{b}_{e, i}$ is a $3 \times 1$ vector, whose coefficients correspond to the linear inequalities defining the relevant half-spaces and supporting hyper-planes of the triangle $\bar{\Omega}_{e, i}$. If the element maximum value ' $\|\boldsymbol{v}\|_{e, i}$ ' is known a priori (through analytically or by means of a rigorous mathematical analysis), then one can use such information in STEP-7 of Algorithm 1. Otherwise, we need to solve equations (4.43a)-(4.43b) using the standard constrained optimization algorithms for small-scale problems [95]. Similarly, equation (4.42b) can be reformulated based on the lines of equations (4.43a)-(4.43b).

4.3.1. Péclet and Damköhler numbers for simplicial meshes. Herein, we shall describe three types of Péclet and Damköhler numbers for simplicial meshes. They are devised based on Theorems 4.3 and 4.4. However, it should be noted that extending it to non-simplicial elements, such as Q4, is not straightforward. This is because in order to construct Péclet and Damköhler numbers for non-simplicial meshes, one needs to obtain mesh restrictions using the global stiffness restriction method. This is beyond the scope of the current paper. 
$\overline{\text { Algorithm } 1 \text { An iterative method to generate an anisotropic } \mathcal{M} \text {-uniform mesh satisfying discrete }}$ principles

1: INPUT: Background mesh $\left(\mathcal{T}_{h, 0}, N e l e_{0}, N v_{0}\right.$, and $\left.N b v_{0}\right)$; anisotropic diffusivity tensor $(\mathbf{D}(\mathbf{x}))$; velocity vector field $(\mathbf{v}(\mathbf{x}))$; linear reaction coefficient $(\alpha(\mathbf{x}))$; maximum number of iterations (MaxIters); piecewise positive scalar element metric constants $\left(\left\{\Theta_{e}\right\}_{e=1}^{\text {Neleo }}\right)$; and a stopping criteria (StopCrit)

- $\mathcal{T}_{h, 0}$ is the initial background triangulation on which an anisotropic mesh generator operates

- $N v_{0}$ and $N b v_{0}$ are correspondingly the total number of vertices and boundary vertices

2: Set the iteration number: $i=0$

3: while (True) do

4: Compute the element average anisotropic diffusivity tensor using a quadrature rule (for example, see References [94])

- $\quad \widetilde{\boldsymbol{D}}_{e, i}:=\frac{1}{\operatorname{meas}\left(\Omega_{e}\right)} \int_{\Omega_{e}} \mathbf{D}(\mathbf{x}) \mathrm{d} \Omega \quad \forall e=1,2, \cdots$, Nele $e_{i}$

5: $\quad$ Compute the element metric tensor by explicitly inverting $\widetilde{\boldsymbol{D}}_{e, i}$

- $\mathcal{M}_{e, i}:=\Theta_{e}\left(\widetilde{\boldsymbol{D}}_{e, i}\right)^{-1} \quad \forall e=1,2, \cdots$, Nele $_{i}$

6: Based on the set of metric tensors $\left\{\mathcal{M}_{e, i}\right\}_{e=1}^{\text {Nele }_{i}}$, compute a new triangulation $\widetilde{\mathcal{T}}_{h, i}$

- $\quad$ Output the new triangulation $\widetilde{\mathcal{T}}_{h, i}$. Corresponding to this $\widetilde{\mathcal{T}}_{h, i}$, we have $\widetilde{N e l e}_{i}, \widetilde{N v_{i}}$, and $\widetilde{N b} v_{i}$

7: $\quad$ Compute the following quantities: $\forall e=1,2, \cdots, \widetilde{\text { Nele }_{i}}$

- $\widetilde{\boldsymbol{D}}_{e, i} ; \Lambda_{\min , \widetilde{\boldsymbol{D}}_{e, i}} ;\|\boldsymbol{v}\|_{e, i}:=\|\boldsymbol{v}(\boldsymbol{x})\|_{\infty, \bar{\Omega}_{e, i}} ;$ and $\|\alpha\|_{e, i}:=\|\alpha(\boldsymbol{x})\|_{\infty, \bar{\Omega}_{e, i}}$

- $\quad$ Need to use a constrained optimization methodology to calculate $\|\boldsymbol{v}\|_{e, i}$ and $\|\alpha\|_{e, i}$ (see Remark 4.6 for more details)

8: $\quad$ if (StopCrit $=$ Anisotropic non-obtuse angle condition) then

9: $\quad$ Check the inequality given by equation (4.15) in Theorem $4.3 \forall e=1,2, \cdots, \widetilde{\text { Nele }}_{i}$

10: $\quad$ if (true) then

11: $\quad$ OUTPUT: The triangulation $\widetilde{\mathcal{T}}_{h, i}$ and corresponding $\left\{\mathcal{M}_{e, i}\right\}_{e=1}^{\text {Nele }_{i}}$. EXIT

12: $\quad$ else

Update $\mathcal{T}_{h, i} \leftarrow \widetilde{\mathcal{T}}_{h, i}, N e l e_{i} \leftarrow \widetilde{N e l e}_{i}, N v_{i} \leftarrow \widetilde{N v_{i}}, N b v_{i} \leftarrow \widetilde{N b v_{i}}$, and $i \leftarrow(i+1)$ end if

end if

if (StopCrit $=$ Generalized Delaunay-type angle condition) then

Check the inequality given by equation (4.17) in Theorem $4.4 \forall e=1,2, \cdots, \widetilde{\text { Nele }_{i}}$

if (true) then

OUTPUT: The triangulation $\widetilde{\mathcal{T}}_{h, i}$ and corresponding $\left\{\mathcal{M}_{e, i}\right\}_{e=1}^{\text {Nele }_{i}}$. EXIT else

Update $\mathcal{T}_{h, i} \leftarrow \widetilde{\mathcal{T}}_{h, i}, N e l e_{i} \leftarrow \widetilde{N e l e}_{i}, N v_{i} \leftarrow \widetilde{N v}_{i}, N b v_{i} \leftarrow \widetilde{N b v_{i}}$, and $i \leftarrow(i+1)$

end if

end if

if $(i>$ MaxIters) then

OUTPUT: The existing triangulation $\widetilde{\mathcal{T}}_{h, i}$ and corresponding $\left\{\mathcal{M}_{e, i}\right\}_{e=1}^{\text {Nele }_{i}}$.

Anisotropic $\mathcal{M}$-uniform triangulation not found in MaxIters. EXIT

end if

end while 
- Element Péclet and Damköhler numbers: Based on Theorem 4.3, one can define the following mesh-based non-dimensional element Péclet $\left(\mathbb{P e}_{\Omega_{e}}\right)$ and Damköhler $\left(\mathbb{D a} \Omega_{e}\right)$ numbers:

$$
\begin{aligned}
\operatorname{Pe}_{\Omega_{e}} & :=\frac{h_{\max , \Omega_{e}}\|\boldsymbol{v}\|_{\infty, \bar{\Omega}_{e}}}{\Lambda_{\min , \widetilde{\boldsymbol{D}}_{\Omega_{e}}}} \\
\operatorname{Da}_{\Omega_{e}} & :=\frac{h_{\max , \Omega_{e}} h_{\mathrm{pumax}, \Omega_{e}}\|\alpha\|_{\infty, \bar{\Omega}_{e}}}{\Lambda_{\min , \widetilde{\boldsymbol{D}}_{\Omega_{e}}}}
\end{aligned}
$$

where the height $h_{\text {pumax }, \Omega_{e}}$ is given as follows:

$0<h_{1} \leq h_{2} \leq \cdots \leq h_{i} \leq \cdots \leq h_{\operatorname{pumax}, \Omega_{e}} \leq h_{\max , \Omega_{e}} \quad \forall i=1,2, \cdots, n d+1, \quad \Omega_{e} \in \mathcal{T}_{h}$

Correspondingly, using equations (4.44a)-(4.44b) in equation (4.15) gives the following (stronger) mesh restriction condition based on Theorem 4.3:

$$
\begin{aligned}
0<\frac{\mathbb{P e}_{\Omega_{e}}}{(n d+1) \cos \left(\beta_{\left.i j, \widetilde{\boldsymbol{D}}_{\Omega_{e}}^{-1}\right)}\right.}+\frac{\mathbb{D} a_{\Omega_{e}}}{(n d+1)(n d+2) \cos \left(\beta_{\left.i j, \widetilde{\boldsymbol{D}}_{\Omega_{e}}^{-1}\right)}\right.} \leq 1 \\
i=\max \text { and } j=\operatorname{pumax}, i \neq j, \forall \Omega_{e} \in \mathcal{T}_{h}
\end{aligned}
$$

- Edge Péclet and Damköhler numbers: In a similar fashion, utilizing Theorem 4.4, one can define the following mesh-based non-dimensional edge Péclet $\left(\mathbb{P e}_{\Omega_{e}, e_{p q}}\right)$ and Damköhler $\left(\mathbb{D a}_{\Omega_{e}, e_{p q}}\right)$ numbers:

$$
\begin{aligned}
\operatorname{Pe}_{\Omega_{e}, e_{p q}} & :=\frac{\operatorname{meas}\left(\Omega_{e}\right)\|\boldsymbol{v}\|_{\infty, \bar{\Omega}_{e}}}{h_{q, \Omega_{e}} \sqrt{\operatorname{det}\left[\widetilde{\boldsymbol{D}}_{\Omega_{e}}\right]}} \\
\mathbb{D} a_{\Omega_{e}, e_{p q}} & :=\frac{\operatorname{meas}\left(\Omega_{e}\right)\|\alpha\|_{\infty, \bar{\Omega}_{e}}}{\sqrt{\operatorname{det}\left[\widetilde{\boldsymbol{D}}_{\Omega_{e}}\right]}}
\end{aligned}
$$

Correspondingly, using equations (4.47a)-(4.47b) in equation (4.17) gives the following (weaker) mesh restriction condition based on Theorem 4.4:

$$
\begin{aligned}
0 & <\frac{1}{2 \pi}\left[\beta_{p q, \widetilde{\boldsymbol{D}}_{\Omega_{e}}^{-1}}+\beta_{p q, \widetilde{\boldsymbol{D}}_{\Omega_{e}^{\prime}}^{-1}}\right] \\
& +\frac{1}{2 \pi} \operatorname{arccot}\left(\sqrt{\frac{\operatorname{det}\left[\widetilde{\boldsymbol{D}}_{\Omega_{e}^{\prime}}\right]}{\operatorname{det}\left[\widetilde{\boldsymbol{D}}_{\Omega_{e}}\right]}}\left(\cot \left(\beta_{p q, \widetilde{\boldsymbol{D}}_{\Omega_{e}^{\prime}}^{-1}}\right)-\frac{2 \mathrm{Pe}_{\Omega_{e}^{\prime}, e_{p q}}}{3}-\frac{\mathbb{D a}_{\Omega_{e}^{\prime}, e_{p q}}}{6}\right)-\frac{2 \mathrm{Pe}_{\Omega_{e}, e_{p q}}}{3}-\frac{\mathbb{D a}_{\Omega_{e}, e_{p q}}}{6}\right) \\
& +\frac{1}{2 \pi} \operatorname{arccot}\left(\sqrt{\frac{\operatorname{det}\left[\widetilde{\boldsymbol{D}}_{\Omega_{e}}\right]}{\operatorname{det}\left[\widetilde{\boldsymbol{D}}_{\Omega_{e}^{\prime}}\right]}}\left(\cot \left(\beta_{p q, \widetilde{\boldsymbol{D}}_{\Omega_{e}}^{-1}}\right)-\frac{2 \mathbb{P} e_{\Omega_{e}, e_{p q}}}{3}-\frac{\mathbb{D a}_{\Omega_{e}, e_{p q}}}{6}\right)-\frac{2 \mathrm{Pe}_{\Omega_{e}^{\prime}, e_{p q}}}{3}-\frac{\mathbb{D a}_{\Omega_{e}^{\prime}, e_{p q}}}{6}\right) \\
& \leq 1
\end{aligned}
$$

- Global mesh Péclet and Damköhler numbers: On sufficiently fine h-refined simplicial meshes (which confirm to Theorem 4.3), one can define a global mesh Péclet $\left(\mathbb{P e}_{h}\right)$ and 
Damköhler $\left(\mathbb{D a}_{h}\right)$ numbers by modifying equations (4.44a)-(4.44b) as follows:

$$
\begin{aligned}
& \mathbb{P e}_{h}:= \frac{h \max _{\Omega_{e} \in \mathcal{T}_{h}}\left[\|\boldsymbol{v}\|_{\infty, \bar{\Omega}_{e}}\right]}{\min _{\Omega_{e} \in \mathcal{T}_{h}}\left[\Lambda_{\left.\min , \widetilde{\boldsymbol{D}}_{\Omega_{e}}\right]}\right.} \\
& \mathbb{D a} \mathrm{a}_{h}:=\frac{h_{\Omega_{e} \in \mathcal{T}_{h}}^{2}\left[\|\alpha\|_{\infty, \bar{\Omega}_{e}}\right]}{\min _{\Omega_{e} \in \mathcal{T}_{h}}\left[\Lambda_{\left.\min , \widetilde{\boldsymbol{D}}_{\Omega_{e}}\right]}\right.}
\end{aligned}
$$

Conservatively, equation (4.46) can be modified to give a (stronger) global mesh restriction condition based on Theorem 4.3:

$$
\begin{gathered}
0<\frac{\mathbb{P e}_{h}}{(n d+1) \min _{\Omega_{e} \in \mathcal{T}_{h}}\left[\cos \left(\beta_{\left.i j, \widetilde{\boldsymbol{D}}_{\Omega_{e}}^{-1}\right)}\right]\right.}+\frac{\mathbb{D a} a_{h}}{(n d+1)(n d+2) \min _{\Omega_{e} \in \mathcal{T}_{h}}\left[\cos \left(\beta_{i j, \widetilde{\boldsymbol{D}}_{\Omega_{e}}^{-1}}\right)\right]} \leq 1 \\
i=\max \text { and } j=\operatorname{pumax}, i \neq j
\end{gathered}
$$

One should note that equation (4.50) can provide a useful a priori (conservative) estimate on $h$ for constructing highly refined simplicial meshes.

4.3.2. Physics-based Péclet and Damköhler numbers. For isotropic diffusivity, it is wellknown that the following three physics-based non-dimensional numbers can be used to understand the qualitative nature of the solutions $[\mathbf{5}, \mathbf{9 6}, \mathbf{9 7}]$ :

$$
\begin{aligned}
& \mathbb{P e}_{D}:=\frac{\max _{\boldsymbol{x} \in \bar{\Omega}}\left[\|\mathbf{v}(\mathbf{x})\|_{\infty}\right] L}{\min _{\boldsymbol{x} \in \bar{\Omega}}[D(\mathbf{x})]} \text { Péclet number } \\
& \mathbb{D a I}:=\frac{\max _{\boldsymbol{x} \in \bar{\Omega}}\left[\|\alpha(\mathbf{x})\|_{\infty}\right] L}{\max _{\boldsymbol{x} \in \bar{\Omega}}\left[\|\mathbf{v}(\mathbf{x})\|_{\infty}\right]} \quad \text { Damköhler number of first kind } \\
& \mathbb{D a} \mathrm{aII}, D:=\frac{\max _{\boldsymbol{x} \in \bar{\Omega}}\left[\|\alpha(\mathbf{x})\|_{\infty}\right] L^{2}}{\min _{\boldsymbol{x} \in \bar{\Omega}}[D(\mathbf{x})]} \quad \text { Damköhler number of second kind }
\end{aligned}
$$

where $L$ is the characteristic length of the domain and $\|\bullet\|_{\infty}$ is the standard max-norm/infinitynorm for vectors [56]. However, it should be noted that the above three non-dimensional numbers are not independent, as they satisfy the relation $\mathbb{D} \mathrm{a}_{\mathrm{II}, D}=\mathbb{P e}_{D} \mathbb{D}$ a. . Physically, the non-dimensional Péclet number characterizes the relative dominance of advection as compared to diffusion processes. For larger Péclet numbers, the advection process dominates and for smaller Péclet numbers, the diffusion process dominates.

In the literature on chemically reacting systems (e.g., see [98, Table 22.2.1]), there exists various Damköhler numbers that relate progress of chemical reactions with respect to mixing, diffusivity, reaction coefficient, thermal effects, and advection. The Damköhler number of first-kind, $\mathbb{D} a_{I}$, gives information about the relative influence of a linear reaction coefficient to that of advection. For small values of $\mathbb{D}$ aI, advection progresses much faster than decay of the chemical species and has the opposite effect for large values of the number. The Damköhler number of second kind, Da $\mathrm{III}, D_{D}$, gives information related to the progress of chemical reaction with respect to diffusion. Based on the chemical system under consideration, these three non-dimensional numbers dictate how the 
reaction, advection, and diffusion interact with each other. On the other hand, one should note that extending it to anisotropic diffusivity is not straightforward. Motivated by equations (4.49a)(4.49b), a way to define physics-based non-dimensional numbers for anisotropic diffusivity tensor $\mathbf{D}(\mathbf{x})$ is as follows:

$$
\begin{aligned}
\mathbb{P e}_{\mathbf{D}}:=\frac{\max _{\boldsymbol{x} \in \bar{\Omega}}\left[\|\mathbf{v}(\mathbf{x})\|_{\infty}\right] L}{\min _{\boldsymbol{x} \in \bar{\Omega}}\left[\Lambda_{\min , \mathbf{D}(\mathbf{x})]}\right.} \quad \text { Péclet number } \\
\mathbb{D} \mathrm{a}_{\mathrm{II}, \mathbf{D}}:=\frac{\max _{\boldsymbol{x} \in \bar{\Omega}}\left[\|\alpha(\mathbf{x})\|_{\infty}\right] L^{2}}{\min _{\boldsymbol{x} \in \bar{\Omega}}\left[\Lambda_{\min , \mathbf{D}(\mathbf{x})]} \quad\right. \text { Damköhler number of second kind }}
\end{aligned}
$$

where $\Lambda_{\min , \mathbf{D}(\mathbf{x})}$ is the minimum eigenvalue of $\mathbf{D}(\mathbf{x})$ at a given point $\mathbf{x}$. The Damköhler number of first kind, $\mathbb{D}$ aI, given by equation (4.51b) remains the same as it does not depend on diffusivity tensor $\mathbf{D}(\mathbf{x})$. Alternatively, inspired by equations (4.47a)-(4.47b), one can define a different set of physics-based Péclet and Damköhler numbers. These are given as follows:

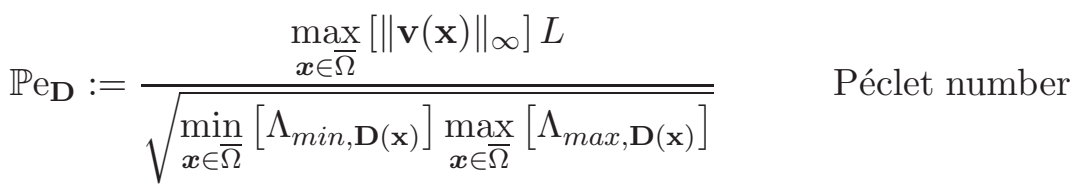

$$
\begin{aligned}
& \mathbb{D a} \mathrm{a}_{\mathrm{II}, \mathbf{D}}:=\frac{\max _{\boldsymbol{x} \in \bar{\Omega}}\left[\|\alpha(\mathbf{x})\|_{\infty}\right] L^{2}}{\sqrt{\min _{\boldsymbol{x} \in \bar{\Omega}}\left[\Lambda _ { \operatorname { m i n } , \mathbf { D } ( \mathbf { x } ) ] } \operatorname { m a x } _ { \boldsymbol { x } \in \overline { \Omega } } \left[\Lambda_{\max , \mathbf{D}(\mathbf{x})]}\right.\right.}} \quad \text { Damköhler number of second kind }
\end{aligned}
$$

where $\Lambda_{\max , \mathbf{D}(\mathbf{x})}$ is the maximum eigenvalue of $\mathbf{D}(\mathbf{x})$ at a given point $\mathbf{x}$. One should note that both these sets of non-dimensional numbers (given by equations (4.52a)-(4.52b) and (4.53a)-(4.53b)) are perfectly valid, as anisotropy in diffusivity tensor can introduce multiple ways of defining physics-based Péclet and Damköhler numbers. Now, we shall present various numerical examples to demonstrate the pros and cons of the mesh restrictions approach.

4.3.3. Test problem \#1: Transport in fractured media. This test problem has profound impact in simulating the transport of chemical species in fractured media [99]. The numerical simulations, using the Delaunay-Voronoi triangulation (with MaxIters $=50$ ) for different cases of diffusivity, velocity field, and linear reaction coefficient, are presented in Figure 15. Homogeneous Dirichlet boundary conditions are prescribed on the sides of the fractured domain; $c^{\mathrm{p}}(\mathbf{x})=1.0$ on the left set of fracture lines and $c^{\mathrm{p}}(\mathbf{x})=2.5$ on the right set of fracture lines. The volumetric source $f(\mathbf{x})$ is zero inside the fractured domain. Diffusivity is assumed to isotropic and scalar, whose value is given by $D(\mathbf{x})=10^{-3}$. We perform numerical simulations for three different cases of velocity field and linear reaction coefficient, which are given by $\mathbf{v}(\mathbf{x})=(0.0,0.0)$ and $\alpha(\mathbf{x})=0.0$, $\mathbf{v}(\mathbf{x})=(0.1,1.0)$ and $\alpha(\mathbf{x})=0.0$, and $\mathbf{v}(\mathbf{x})=(0.1,1.0)$ and $\alpha(\mathbf{x})=1.0$.

From Figure 15, it can be inferred that we need highly refined DMP-based triangular meshes to obtain physically meaningful values for concentration for large values of edge Péclet and Damköhler numbers. The white region in the figures indicates the area in which the value of concentration is negative and also violated the maximum constraint. The coarse Delaunay-Voronoi mesh obtained using the open source mesh generator Gmsh satisfies NC and DMPs in the case of pure diffusion. But, this is not true for $\mathrm{AD}$ and $\mathrm{ADR}$ cases. In such scenarios, it produces unphysical values for 
the concentration field. Moreover, the percentage of nodes that have violated NC and maximum constraint is also very high.

Quantitatively, Tables 1 and 2 provide more details pertinent to the violations in NC and DMPs for $\mathrm{AD}$ and $\mathrm{ADR}$ cases. It should be noted that the decrease in unphysical values of concentration is not monotonic. This is because the generalized Delaunay-type angle condition, in general, does not ensure uniform convergence for diffusion-type equations in $L^{\infty}$ norm (for example, in case of pure isotropic diffusion, see the mesh restriction result by Ciarlet and Raviart [12]). Figure 16 shows that the weak DMP-based condition is satisfied only for pure diffusion equation. But in all other cases, generalized Delaunay-type condition is violated. In the case of pure diffusion, it should be noted that DMP-based mesh given in Figure 16 is not interiorly connected. Hence, it only satisfies $\operatorname{DWMP}_{\boldsymbol{K}}$, but not $\operatorname{DSMP}_{\boldsymbol{K}}$.

4.3.4. Test problem \#2: Species dispersion in subsurface flows. A pictorial description of the boundary value problem with various parameters is shown in Figure 17. Relevant (coarse) background mesh used and the corresponding DMP-based mesh (with MaxIters $=50$ ) obtained using BAMG are shown in Figure 18. The diffusivity tensor for this problem is taken from the subsurface hydrology literature [100] and is given as follows:

$$
\mathbf{D}_{\text {subsurface }}(\mathbf{x})=\alpha_{T}\|\mathbf{v}\| \mathbf{I}+\frac{\alpha_{L}-\alpha_{T}}{\|\mathbf{v}\|} \mathbf{v} \otimes \mathbf{v}
$$

where $\otimes$ is the tensor product, $\mathbf{I}$ is the identity tensor, $\mathbf{v}$ is velocity vector field of the subsurface flow, and $\alpha_{T}$ and $\alpha_{L}$ are, respectively, transverse and longitudinal diffusivity coefficients with $\alpha_{T}=0.01$ and $\alpha_{L}=0.1$. It should be emphasized that we have neglected advection. Correspondingly, the numerical values for the velocity vector field used to define the diffusion tensor is given by $\mathbf{v}(\mathbf{x})=(1.0,1.0)$. This test problem has importance in simulating diffusion of chemical species in subsurface flows of hydrogeological systems [101-103].

Numerical simulations using these meshes are shown in Figure 19. The white region in this figure depicts the area in which the value of concentration is negative. From Figure 19, it is apparent that the coarse anisotropic triangulation violates the DMPs and NC. This is because the Algorithm 1 did not converge in MaxIters. However, quantitatively, this violation in NC is low as compared to background mesh. Specifically, the minimum concentration and the percentage of nodes that have violated the non-negative constraint on the background mesh is about $-4.8 \times 10^{-5}$ and $2.13 \%$, while these values on the anisotropic triangulation are around $-1.35 \times 10^{-8}$ and $0.34 \%$. Additionally, from Figure 19, it is evident that we need a highly refined DMP-based anisotropic mesh to avoid negative values for concentration. Nevertheless, it should be noted that there is a considerable decrease in the negative values for concentration if a traditionally $h$-refined anisotropic triangulation is used (see Figure 23 and subsubsection 4.3.6 for more details).

4.3.5. Test problem \#3: Contaminant transport in leaky wells. The computational domain is a circle with a hole centered at origin $(0,0)$. The radius of the circular hole and the circular domain are 0.1 and 1.0. Numerical simulations are performed for four different cases of the velocity vector field and linear reaction coefficient, which are given by $\mathbf{v}(\mathbf{x})=(0.0,0.0)$ and $\alpha(\mathbf{x})=0.0, \mathbf{v}(\mathbf{x})=(1.5,1.0)$ and $\alpha(\mathbf{x})=1.0, \mathbf{v}(\mathbf{x})=(5.0,0.5)$ and $\alpha(\mathbf{x})=1.0$, and $\mathbf{v}(\mathbf{x})=(0.0,0.0)$ and $\alpha(\mathbf{x})=1000$. Each case is designed to test a particular aspect. For example, $\mathbf{v}(\mathbf{x})=(5.0,0.5)$ and $\alpha(\mathbf{x})=1.0$ corresponds to advection-dominated ADR problems, while $\mathbf{v}(\mathbf{x})=(0.0,0.0)$ and $\alpha(\mathbf{x})=1000$ corresponds to reaction-dominated diffusion-reaction problems. The diffusivity tensor 
for this problem is given by equations (1.1)-(1.3). Correspondingly, the values for the parameters $d_{\max }, d_{\min }$, and $\theta$ are equal to $1,0.001$, and $\frac{\pi}{3}$.

The computational domain in this test problem has various practical applications related to well design in multi-aquifers and groundwater distribution systems [104-106], understanding emanation of gaseous hydrocarbons through bore-holes in oil and gas reservoirs [107-109], and to study leakage of contaminants (such as $\mathrm{CO}_{2}$, salts, and nitrates) through abandoned wells [110-113]. The background mesh used and the corresponding DMP-based (coarse) mesh obtained using BAMG are shown in Figure 20 (MaxIters $=50)$. For the cases when $\mathbf{v}(\mathbf{x})=(0.0,0.0)$ and $\alpha(\mathbf{x})=0.0$, $\mathbf{v}(\mathbf{x})=(1.5,1.0)$ and $\alpha(\mathbf{x})=1.0$, Algorithm 1 converged in MaxIters. But for $\mathbf{v}(\mathbf{x})=(5.0,0.5)$ and $\alpha(\mathbf{x})=1.0, \mathbf{v}(\mathbf{x})=(0.0,0.0)$ and $\alpha(\mathbf{x})=1000$, Algorithm 1 did not converge in MaxIters. Herein, the DMP-based coarse mesh is composed of needle-type triangles. This is because the ratio of the minimum eigenvalue of $\mathbf{D}(\mathbf{x})$ to its maximum is 0.001 (which is related to the aspect ratio of the sides of the triangle in the DMP-based anisotropic mesh). Moreover, it is evident that the triangles in the mesh are aligned and oriented along the principal axis of the eigenvectors of the diffusivity tensor.

Numerical simulations using these meshes are shown in Figure 21. The white region in the figures (circular annulus) shows the area in which the value of concentration is negative. The minimum concentration and the percentage of nodes that have violated the non-negative constraint for the background mesh are about $-1.67 \times 10^{-2}$ and $30.28 \%$. As the anisotropic mesh is coarse and the Algorithm 1 did not converge in MaxIters for advection-dominated ADR problems and reactiondominated diffusion-reaction problems, the resulting mesh not only violates the non-negative constraint, but also produces spurious oscillations. The minimum concentration and the percentage of nodes that have violated the non-negative constraint for the case when $\mathbf{v}=(5.0,0.5)$ and $\alpha=1.0$ are about $-1.78 \times 10^{-1}$ and $13.47 \%$, whereas for the case when $\mathbf{v}=(0.0,0.0)$ and $\alpha=1000$ are around $-2.79 \times 10^{-1}$ and $20.54 \%$. Hence in both these cases, we need a highly refined DMP-based mesh to avoid negative values for concentration (see subsubsection 4.3.6 for more details). For scenarios when $\mathbf{v}(\mathbf{x})=(0.0,0.0)$ and $\alpha(\mathbf{x})=0.0, \mathbf{v}(\mathbf{x})=(1.5,1.0)$ and $\alpha(\mathbf{x})=1.0$, the coarse DMP-based mesh is interiorly connected and satisfies $\operatorname{DsMP}_{\boldsymbol{K}}\left(\right.$ or $\operatorname{DSMP}_{\boldsymbol{K}}$, when $\left.\alpha(\mathbf{x})=0\right)$.

4.3.6. Issues with DMP-based h-refinement. From the above set of test problems, it is apparent that mesh refinement is needed to avoid spurious node-to-node oscillations and satisfaction of various discrete principles (mainly for the cases when the values of the velocity vector field and linear reaction coefficient are predominant as compared to minimum eigenvalue of diffusivity tensor). In general, within the context of computational geometry and mesh generation literature, there are various methods to generate different types of $h$-refined meshes $[\mathbf{6 1}, \mathbf{6 3}, \mathbf{7 3}]$. However, it is evident from these test problems that we are interested in $h$-refined meshes that conform to either anisotropic non-obtuse angle condition or generalized Delaunay-type angle condition. Herein, we shall study two different and important methodologies that are popular in mesh generation literature and implemented in the open source mesh generators, such as Gmsh and BAMG in FreeFem ++ . Our objective is to understand whether the $h$-refined meshes generated using these mesh generators satisfy DMPs, DCPs, and NC or not.

Traditional $h$-refinement. In this method, an $h$-refined mesh is obtained by splitting each triangle in the coarse mesh into multiple triangles. Based on this methodology, numerical simulations are performed using the traditional $h$-refined meshes (which are derived based on the DMP-based coarse meshes presented in Figures 15, 18, and 20). The resulting concentration profiles for test 
TABLE 1. Fractured domain with isotropic diffusivity: For AD equation

\begin{tabular}{|c|c|c|c|c|}
\hline Delaunay triangulation & \multicolumn{2}{|c|}{ Concentration } & \multicolumn{2}{c|}{ \% of nodes violated } \\
\cline { 2 - 5 }$($ Nv, Nele, $h)$ & Minimum & Maximum & (Non-negative constraint) & (Maximum constraint) \\
\hline$(539,906,0.1)$ & -10.51 & 9.85 & 17.44 & 13.73 \\
$(1564,2826,0.05)$ & -18.26 & 5.86 & 18.22 & 14.19 \\
$(5090,9620,0.025)$ & -4.65 & 5.71 & 16.09 & 13.77 \\
$(18372,35665,0.0125)$ & -2.97 & 5.17 & 12.82 & 12.70 \\
$(69995,137881,0.00625)$ & -1.62 & 3.89 & 8.08 & 8.47 \\
\hline
\end{tabular}

problems \#1, \#2, and \#3, are shown in Figures 22-24. Qualitatively and quantitatively, the following inferences can be drawn from these numerical results:

- Test problem \#1: For isotropic diffusivity, from Figure 22, Table 1, and Table 2, it is apparent that there is a decrease in negative values for concentration and reduction in spurious oscillations.

- Test problem \#2: Qualitatively, based on Figure 23, it can be concluded that there is a considerable decrease in the violation of non-negative constraint. Quantitatively, for this $h$-refined anisotropic triangulation, the minimum concentration and the percentage of nodes that have violated the non-negative constraint is about $-1.15 \times 10^{-11}$ and $0.02 \%$ (which is significantly close to machine epsilon $\epsilon_{\text {mach }} \approx 2.22 \times 10^{-16}$ ).

- Test problem \#3: For the pure anisotropic diffusion case, the coarse anisotropic DMPbased mesh given in Figure 20 satisfies NC and all of the DMPs. However, contrary to this, on traditional $h$-refinement, the resulting anisotropic triangulation produces unphysical negative values and violates the DMPs. Quantitatively, this violation is far from machine epsilon, $\epsilon_{\text {mach }}$. Correspondingly, the minimum concentration and the percentage of nodes that have violated the non-negative constraint is about $-2.7 \times 10^{-1}$ and $28.51 \%$, which is way higher as compared the numerical simulations based on the background mesh.

To summarize, it is clear that traditional h-refinement does not always reduce the unphysical numerical values. Certainly, there is a decrease in negative values for concentration for test problem $\# 1$ and \#2. But, this is not the case for test problem \#3. This is because the methodology to obtain traditional $h$-refinement meshes from a given coarse mesh need not conform to the conditions outlined in either Theorem 4.3 or Theorem 4.4.

Non-traditional $h$-refinement. In this method, a $h$-refined mesh is obtained directly from the background mesh (using Algorithm 1) by change certain parameters related to metric tensor, geometry of the domain, and number of nodes on the boundary of the domain [20, Chapter-5]. It should be noted that this methodology is completely different from the traditional $h$-refinement procedure, as we never generate a coarse DMP-based triangulation and then split the respective mesh elements. However, even this non-traditional $h$-refined mesh is not guaranteed to produce physics-compatible numerical values for concentration (as the mesh generation procedure need not converge in MaxIters). This is evident from the numerical simulations performed on the nontraditional $h$-refined mesh for test problem \#3. The corresponding concentration profile is shown in Figure 25. Quantitatively, the minimum concentration and the percentage of nodes that have violated the non-negative constraint are about $-9.69 \times 10^{-2}$ and $34.11 \%$, which is comparatively similar to that of the traditional $h$-refinement methodology. 
TABLE 2. Fractured domain with isotropic diffusivity: For ADR equation

\begin{tabular}{|c|c|c|c|c|}
\hline Delaunay triangulation & \multicolumn{2}{|c|}{ Concentration } & \multicolumn{2}{c|}{ \% of nodes violated } \\
\cline { 2 - 5 }$($ Nv, Nele, $h)$ & Minimum & Maximum & (Non-negative constraint) & (Maximum constraint) \\
\hline$(539,906,0.1)$ & -9.64 & 4.53 & 14.29 & 8.16 \\
$(1564,2826,0.05)$ & -1.54 & 3.57 & 18.03 & 1.53 \\
$(5090,9620,0.025)$ & -4.47 & 3.30 & 16.01 & 0.29 \\
$(18372,35665,0.0125)$ & -2.95 & 2.56 & 12.89 & 0.04 \\
$(69995,137881,0.00625)$ & -1.62 & 2.50 & 8.06 & 0.00 \\
\hline
\end{tabular}

TABLE 3. Errors in local and global species balance: For pure isotropic and anisotropic diffusion equation.

\begin{tabular}{|c|c|c|c|c|c|}
\hline \multirow{2}{*}{$h$} & \multicolumn{2}{|c|}{ Test problem \#1 } & \multirow{2}{*}{$h$} & \multicolumn{2}{|c|}{ Test problem \#2 } \\
\cline { 2 - 3 } \cline { 5 - 5 } & Local error (abs. max.) & Global error & & Local error (abs. max.) & Global error \\
\hline 0.1 & $1.80 \times 10^{-3}$ & $1.53 \times 10^{-2}$ & 0.08 & $5.75 \times 10^{-4}$ & $-1.44 \times 10^{-4}$ \\
0.05 & $4.66 \times 10^{-4}$ & $1.23 \times 10^{-2}$ & 0.042 & $1.47 \times 10^{-4}$ & $-1.38 \times 10^{-4}$ \\
0.025 & $3.48 \times 10^{-4}$ & $8.48 \times 10^{-3}$ & 0.028 & $7.34 \times 10^{-5}$ & $-1.12 \times 10^{-4}$ \\
0.0125 & $7.14 \times 10^{-4}$ & $7.82 \times 10^{-3}$ & 0.0135 & $1.59 \times 10^{-5}$ & $-6.83 \times 10^{-5}$ \\
0.00625 & $6.75 \times 10^{-4}$ & $5.38 \times 10^{-3}$ & 0.0075 & $5.46 \times 10^{-6}$ & $-4.36 \times 10^{-5}$ \\
\hline
\end{tabular}

4.3.7. Errors in local and global species balance. It is well-known that without using a post-processing method, the discrete standard single-field Galerkin formulation does not possess local and global conservation properties $[\mathbf{4 6 , 1 1 4}]$. In finite element literature, there are various postprocessing methods to quantify the errors incurred in satisfying local and global species balance [5, 96,115,116]. Herein, we shall use Herrmann's method of optimal sampling [116, Subsection 15.2.2], which is a popular post-processing technique to obtain derived quantities from primary variables (such as the concentration variable in single-field finite element formulations). In the context of recovery-based error estimators [62], this post-processing method is also known as traditional global smoothing method [115, Subsection 9.9]. To summarize, this method involves minimizing a unconstrained least-squares flux functional to obtain nodal flux vectors. Then, using these flux vectors, local and global species balance errors are computed.

Qualitatively, the contours corresponding to local species balance errors for test problems \#1, $\# 2$, and \#3 on coarse DMP-based meshes are shown in Figure 26. From this figure, it is clear that test problem \#3 exhibits considerable errors in satisfying local species balance. Quantitatively, Table 3 provides local and global species balance errors on traditional $h$-refined meshes for test problems \#1 and \#2. From this table, it can be concluded that the decrease in these errors is slow. Hence, there is need for locally conservative DMP-preserving finite element methods.

REMARK 4.7. It should be noted that there are various ways in which one can develop locally conservative global smoothing methods. For example, this can be achieved by constructing a constrained monotonic regression based method (following the procedure outlined by Burdakov et al. [46]) to obtain a conservative flux vector. However, as discussed in Section 1 and Section 3, it should be note that such type of post-processing methods are not variationally consistent and refutes the purpose of developing physics-compatible DMP-based meshes. 
4.4. Sufficient conditions for a rectangular element. For the sake of illustration, consider a rectangular element whose vertices are located at $(0,0),(a, 0),(a, b)$, and $(0, b)$. Our objective is to derive conditions on $a$ and $b$, such that the local stiffness matrix is weakly diagonally dominant. Herein, we consider a pure anisotropic diffusion equation and derive restrictions on the coordinates of the rectangular element. Based on the lines of the local stiffness restriction method outlined in subsubsection 4.2.1, the local stiffness matrix for the case when diffusivity $\mathbf{D}(\mathbf{x})=\left(\begin{array}{ll}D_{x x} & D_{x y} \\ D_{x y} & D_{y y}\end{array}\right)$ is anisotropic and constant in $\Omega$ is given as follows:

$$
\boldsymbol{K}_{e}=\left(\begin{array}{cccc}
\frac{b D_{x x}}{3 a}+\frac{D_{x y}}{2}+\frac{a D_{y y}}{3 b} & -\frac{b D_{x x}}{3 a}+\frac{a D_{y y}}{6 b} & \frac{b D_{x x}}{6 a}-\frac{a D_{y y}}{3 b} & -\frac{b D_{x x}}{6 a}-\frac{D_{x y}}{2}-\frac{a D_{y y}}{6 b} \\
-\frac{b D_{x x}}{3 a}+\frac{a D_{y y}}{6 b} & \frac{b D_{x x}}{3 a}-\frac{D_{x y}}{2}+\frac{a D_{y y}}{3 b} & -\frac{b D_{x x}}{6 a}+\frac{D_{x y}}{2}-\frac{a D_{y y}}{6 b} & \frac{b D_{x x}}{6 a}-\frac{a D_{y y}}{3 b} \\
\frac{b D_{x x}}{6 a}-\frac{a D_{y y}}{3 b} & -\frac{b D_{x x}}{6 a}+\frac{D_{x y}}{2}-\frac{a D_{y y}}{6 b} & \frac{b D_{x x}}{3 a}-\frac{D_{x y}}{2}+\frac{a D_{y y}}{3 b} & -\frac{b D_{x x}}{3 a}+\frac{a D_{y y}}{6 b} \\
-\frac{b D_{x x}}{6 a}-\frac{D_{x y}}{2}-\frac{a D_{y y}}{6 b} & \frac{b D_{x x}}{6 a}-\frac{a D_{y y}}{3 b} & -\frac{b D_{x x}}{3 a}+\frac{a D_{y y}}{6 b} & \frac{b D_{x x}}{3 a}+\frac{D_{x y}}{2}+\frac{a D_{y y}}{3 b}
\end{array}\right)
$$

Condition No-7. From AM-GM inequality, we have the following relation:

$$
\frac{b D_{x x}}{3 a}+\frac{a D_{y y}}{3 b} \geq \frac{2}{3} \sqrt{D_{x x} D_{y y}}>\frac{2}{3}\left|D_{x y}\right| \geq \frac{1}{2}\left|D_{x y}\right|
$$

which implies that the positive diagonal entries: $\left(\boldsymbol{K}_{e}\right)_{i i}>0 \quad \forall i=1,2,3,4$, is trivially satisfied.

Condition No-8. Non-positive off-diagonal entries: $\left(\boldsymbol{K}_{e}\right)_{i j} \leq 0 \quad \forall i \neq j$ where $i=1,2,3,4$, and $j=1,2,3,4$, needs to be satisfied. For instance, when $i=1$ and $j=2,3,4$, this restriction gives the following relations:

$$
\begin{aligned}
& \sqrt{\frac{D_{x x}}{2 D_{y y}}} \leq \frac{a}{b} \leq \sqrt{\frac{2 D_{x x}}{D_{y y}}} \\
& -\frac{b D_{x x}}{6 a}-\frac{D_{x y}}{2}-\frac{a D_{y y}}{6 b} \leq 0
\end{aligned}
$$

Additionally, we should also satisfy the restrictions imposed by equations (4.30a)-(4.30c) on diffusivity tensor. In a similar fashion, one can derive restrictions for other combinations of $i$ and $j$.

Condition No-9. Weak diagonal dominance of rows: $\left|\left(\boldsymbol{K}_{e}\right)_{i i}\right| \geq \sum_{i \neq j}\left|\left(\boldsymbol{K}_{e}\right)_{i j}\right| \quad \forall i, j$ where $i=1,2,3,4$, and $j=1,2,3,4$, is trivially satisfied if Condition- 7 and Condition- 8 are met. This is because from equation (4.55), it is evident that $\left(\boldsymbol{K}_{e}\right)_{i i}+\sum_{i \neq j}\left(\boldsymbol{K}_{e}\right)_{i j}=0 \quad \forall i, j$ where $i=1,2,3,4$, and $j=1,2,3,4$.

Finally, as explained in subsubsection 4.2.1, extending the local stiffness restriction approach to incorporate advection and linear reaction is straightforward and shall not be dealt with to save space. Moreover, it is easy to construct mesh restrictions for any set of arbitrary coordinates of a quadrilateral element using symbolic packages like Mathematica. But the resulting inequalities will be more complex to analyze mathematically and visualize graphically.

\section{CONCLUDING REMARKS AND OPEN QUESTIONS}

We outlined a general procedure to obtain the restrictions that are needed for a computational grid to satisfy various mathematical principles - comparison principles, maximum principles, and the non-negative constraint. We illustrated the workings of this procedure by obtaining the mesh 
restrictions for T3 and Q4 finite elements. The procedure is, however, equally applicable to other low-order finite elements.

First, we critiqued three different approaches to satisfy maximum principles, comparison principles, and non-negative constraint for a general linear second-order elliptic equation. A pictorial description of a generic relationship between DMPs, DCPs, and NC based on a Venn diagram is proposed. This sketch helps to easily relate the space of solutions obtained using mesh restrictions, non-negative numerical formulations, and post-processing methods. We then presented necessary and sufficient conditions on the stiffness matrix $\boldsymbol{K}$ to meet the mathematical properties. Using these conditions, we derived stronger and weaker mesh restrictions for T3 element. The stronger mesh restriction corresponds to the anisotropic non-obtuse angle condition while the weaker one corresponds to the generalized Delaunay-type angle condition. Motivated by these mesh restriction conditions, different kinds of Péclet and Damköhler numbers are proposed for advectivediffusive-reactive systems when diffusivity is anisotropic.

For isotropic diffusivity, we established that acute-angled or right-angled triangle is sufficient to satisfy discrete principles. However, for anisotropic diffusivity, we showed that in order to satisfy DMPs, DCPs, and NC, all the dihedral angles of a simplex measured in the metric of $\widetilde{\boldsymbol{D}}_{\Omega_{e}}^{-1}$ have to be either $\mathcal{O}\left(h\|\boldsymbol{v}\|_{\infty, \mathcal{T}_{h}}+h^{2}\|\alpha\|_{\infty, \mathcal{T}_{h}}\right)$ acute/non-obtuse or $\mathcal{O}\left(h\|\boldsymbol{v}\|_{\infty, \mathcal{T}_{h}}+h^{2}\|\alpha\|_{\infty, \mathcal{T}_{h}}\right)$ Delaunay. Pictorially, this means that the feasible region for T3 and Q4 elements to satisfy various discrete principles is based on a metric tensor whose components are a function of anisotropic diffusivity with respect to a suitable coordinate system. Then, an anisotropic metric tensor and an iterative algorithm to generate various types of DMP-based triangulations are described. Different numerical examples and respective DMP-based triangular meshes are presented for different types of $\mathbf{D}(\mathbf{x})$ to demonstrate the pros and cons of imposing mesh restrictions. Furthermore, the errors incurred in satisfying local and global species balance are documented. Based on these numerical experiments, the following inferences can be drawn:

(C1) For pure isotropic or anisotropic diffusion equation, a coarse DMP-based triangulation is sufficient to satisfy various discrete principles. However, for advection-dominated and reactiondominated scenarios, we need a highly refined DMP-preserving computational mesh to obtain non-negative solutions.

(C2) Existing traditional and non-traditional methods of $h$-refinement may not guarantee the satisfaction of DMPs, DCPs, and NC always.

(C3) On coarse DMP-based meshes, errors incurred in satisfying local and global species balance for highly anisotropic diffusion-type problems is considerable due to the skewed nature of the mesh elements. Moreover, the decrease in local and global species balance errors upon $h$-refinement is slow.

(C4) DMP-based meshes change as one alters the underlying anisotropic diffusivity tensor.

In the light of the recent developments and motivated by the above discussions, we have chosen to emphasize on the following four open problems that we consider particularly interesting in view of their mathematical richness, numerical challenges, and potential applications:

(OP1) In this paper, all the meshes used in the numerical examples are of Delaunay-type. This is because most of the existing open source mesh generators such as BAMG, Gmsh, Triangle, BL2D, Mmg3d, and CGALmesh are Delaunay. Recently, Erten and Üngör [15-17] have developed a non-obtuse/acute angled mesh generator called aCute by modifying Triangle. However, aCute is restricted to 2D and Eucledian metric tensors. Hence, such a software 
can only be used to satisfy discrete principles for problems involving heterogeneous isotropic diffusivity. Having an anisotropic non-obtuse/acute $\mathcal{M}$-uniform meshing software would be of great importance, as the numerical solutions obtained from these meshes not only satisfy DMPs, DCPs, and NC, but also converge uniformly (an attractive aspect in finite element analysis [12]). To date, there is no such mesh generator. Developing such a software will have a profound impact on obtaining physically meaningful numerical solutions for diffusiontype equations.

(OP2) For advection-dominated and reaction-dominated advection-diffusion-reaction problems, mesh refinement that adheres to DMPs is needed to obtain stable and sufficiently accurate numerical solutions. As described in (C2), not every method of $h$-refinement is DMPpreserving. Hence, a consistent way of generating a DMP-based $h$-refined mesh (that satisfies Generalized Delaunay-type angle condition) is still unresolved.

(OP3) From (C3), it is apparent that local and global mass conservation property is needed. An approach to preserve such a property without violating DMPs, DCPs, and NC, is to obtain mesh restrictions for mixed Galerkin formulation based on lowest-order Raviart-Thomas spaces. Recently, Huang and Wang [117] have developed a methodology to satisfy DMPs for a class of locally conservative weak Galerkin methods using lowest-order Raviart-Thomas spaces. However, this methodology is limited to pure anisotropic steady-state diffusion equation in two-dimensions. Hence, a mesh restriction based method to satisfy different discrete principles, local species balance, and global species balance for anisotropic advection-diffusion-reaction equations thus far is unsolved.

(OP4) In order to construct DMP-based meshes for low-order non-simplicial finite elements such as Q4, from subsection 4.4, it is evident that stronger and weaker mesh conditions are needed. So far, there are no mesh restriction theorems analogous to simplicial meshes that can provide a general framework to construct non-simplicial meshes for anisotropic diffusivity tensors. Theoretically and numerically, it would be very interesting and informative to have a comparative study on the performance of simplicial vs. non-simplicial DMP-based meshes for various benchmark problems discussed in Section 4.

Nevertheless, due to enormous research activity in the field of advection-diffusion-reaction equations, it is impossible to list every open question on preserving DCPs, DMPs, and NC. To conclude, the research findings in this paper will be invaluable to the research community and finite element practitioners in two respects. First, it will guide the existing users on the restrictions to be placed on the computational mesh to meet important mathematical properties like maximum principles, comparison principles, and the non-negative constraint. Second, for complex geometries and highly anisotropic media, this study has clearly shown that placing restrictions on computational grids may not always be a viable approach to achieve physically meaningful non-negative solutions. We hope that this research work will motivate researchers to develop new methodologies for advective-diffusive-reactive systems that satisfy local and global species balance, comparison principles, maximum principles, and the non-negative constraint on coarse general computational grids.

\section{ACKNOWLEDGMENTS}

The authors acknowledge the support from the DOE Nuclear Energy University Programs (NEUP). The opinions expressed in this paper are those of the authors and do not necessarily 
reflect that of the sponsors. The authors are grateful to the developers of the open source scientific software packages: BAMG, FreeFem++, and Gmsh.

\section{References}

[1] J. Crank. The Mathematics of Diffusion. Clarendon press, Oxford, second edition, 1975.

[2] Z. Mei. Numerical Bifurcation Analysis for Reaction-Diffusion Equations. Springer-Verlag, New York, USA, 2000.

[3] M. Aoki. Modeling Aggregate Behavior and Fluctuations in Economics: Stochastic Views of Interacting Agents. Cambridge University Press, Cambridge, UK, 2004.

[4] C. V. Pao. Nonlinear Parabolic and Elliptic Equations. Springer-Verlag, New York, USA, 1993.

[5] P. M. Gresho and R. L. Sani. Incompressible Flow and the Finite Element Method: Advection-Diffusion, volume 1. John Wiley \& Sons, Inc., Chichester, UK, 2000.

[6] ABAQUS/CAE/Standard, Version 6.8-3. Simulia, Providence, Rhode Island, www.simulia.com, 2009.

[7] ANSYS, Version 14.5. ANSYS, Inc., Canonsburg, Pennsylvania, www.ansys.com, 2012.

[8] COMSOL Multiphysics User's Guide, Version 4.3a. COMSOL, Inc., Burlington, Massachusetts, www.comsol.com, 2012.

[9] MATLAB 2012a. The MathWorks, Inc., Natick, Massachusetts, USA, 2012.

[10] W. Hackbusch. Multi-Grid Methods and Applications. Springer Series in Computational Mathematics. SpringerVerlag, New York, USA, 2003.

[11] K. B. Nakshatrala, M. K. Mudunuru, and A. J. Valocchi. A numerical framework for diffusion-controlled bimolecular-reactive systems to enforce maximum principles and the non-negative constraint. Journal of Computational Physics, 253:278-307, 2013.

[12] P. G. Ciarlet and P-A. Raviart. Maximum principle and uniform convergence for the finite element method. Computer Methods in Applied Methods and Engineering, 2:17-31, 1973.

[13] E. Vanderzee, A. N. Hirani, D. Guoy, and E. A. Ramos. Well-centered triangulation. SIAM Journal on Scientific Computing, 31:4497-4523, 2010.

[14] S. J. Owen. A survey of unstructured mesh generation technology. In 7th International Meshing Roundtable, Sandia National Laboratory, pages 239-267, 1998.

[15] H. Erten and A. Üngör. Computing acute and non-obtuse triangulations. 19th Canadian Conference on Computational Geometry, pages 205-208, 2007.

[16] H. Erten and A. Üngör. Computing triangulations without small and large angles. In Sixth International Symposium on Voronoi Diagrams, IEEE, pages 192-201, 2009.

[17] H. Erten and A. Üngör. Quality triangulations with locally optimal Steiner points. SIAM Journal on Scientific Computing, 31:2103-2130, 2009.

[18] J. R. Shewchuk. Triangle: Engineering a 2D Quality Mesh Generator and Delaunay Triangulator. In Ming C. Lin and Dinesh Manocha, editors, Applied Computational Geometry: Towards Geometric Engineering, volume 1148 of Lecture Notes in Computer Science, pages 203-222. Springer-Verlag, May 1996. From the First ACM Workshop on Applied Computational Geometry.

[19] F. Hecht. BAMG: Bidimensional Anisotropic Mesh Generator. Technical Report, INRIA, Rocquencourt, Website: http://www.ann.jussieu.fr/hecht/ftp/bamg/bamg.pdf, 2006.

[20] F. Hecht, S. Auliac, O. Pironneau, J. Morice, A. L. Hyaric, and K. Ohtsuka. FreeFem++. URL: http://www.freefem.org/ff++/, third edition, 2014. Version 3.26-2.

[21] F. Hecht. New development in FreeFem++. Journal of Numerical Mathematics, 20:251-266, 2012.

[22] P. Laug and H. Borouchaki. The BL2D mesh generator: Beginner's guide, user's and programmer's manual. Technical Report, Website: http://hal.archives-ouvertes.fr/docs/00/06/99/77/PDF/RT-0194.pdf, 1996.

[23] C. Dobrzynski. Mmg3d: User Guide. Technical Report, Website: http://hal.inria.fr/docs/00/68/18/13/PDF/RT422.pdf, 2012.

[24] P. Alliez, D. C.-Steiner, O. Devillers, B. Lévy, and M. Desbrun. Anisotropic polygonal remeshing. ACM Transactions on Graphics, 22:485-493, 2003.

[25] J.-D. Boissonnat, C. Wormser, and M. Yvinec. Locally uniform anisotropic meshing. In Proceedings of the twenty-fourth annual symposium on Computational geometry, ACM, pages 270-277, 2008. 
[26] J.-D. Boissonnat, J.-P. Pons, and M. Yvinec. From segmented images to good quality meshes using delaunay refinement. In F. Nielsen, editor, Emerging Trends in Visual Computing, volume 5416 of Lecture Notes in Computer Science, pages 13-37, Berlin, Heidelberg, 2009. Springer.

[27] J.-D. Boissonnat, C. Wormser, and M. Yvinec. Anisotropic Delaunay mesh generation. HAL: inria-00615486, version 1, 2011.

[28] X. Li and W. Huang. An anisotropic mesh adaptation method for the finite element solution of heterogeneous anisotropic diffusion problems. Journal of Computational Physics, 229:8072-8094, 2010.

[29] C. Lu, W. Huang, and J. Qiu. Maximum principle in linear finite element approximations of anisotropic diffusionconvection-reaction problems. arXiv:1201.3564, 2012.

[30] W. Huang. Sign-preserving of principal eigenfunctions in P1 finite element approximation of eigenvalue problems of second-order elliptic operators. arXiv:1306.1987, 2013.

[31] P. G. Ciarlet. Discrete maximum principle for finite-difference operators. Aequationes Mathematicae, 4:338-352, 1970.

[32] R. Varga. On a discrete maximum principle. SIAM Journal on Numerical Analysis, 3:355-359, 1966.

[33] F. Brezzi, K. Lipnikov, and M. Shashkov. Convergence of the mimetic finite difference method for diffusion problems on polyhedral meshes. SIAM Journal on Numerical Analysis, 43:1872-1896, 2005.

[34] K. Lipnikov, G. Manzini, and D. Svyatskiy. Analysis of the monotonicity conditions in the mimetic finite difference method for elliptic problems. Journal of Computational Physics, 230:2620-2642, 2011.

[35] C. Le Potier. A nonlinear finite volume scheme satisfying maximum and minimum principles for diffusion operators. International Journal of Finite Volumes, 6(2), 2009.

[36] J. M. Nordbotten, I. Aavatsmark, and G. T. Eigestad. Monotonicity of control volume methods. Numerische Mathematik, 106:255-288, 2007.

[37] J. Droniou and C. Le Potier. Construction and convergence study of schemes preserving the elliptic local maximum principle. SIAM Journal of Numerical Analysis, 49:459-490, 2011.

[38] E. Burman and A. Ern. Stabilized Galerkin approximation of convection-diffusion-reaction equations: Discrete maximum principle and convergence. Mathematics of Computation, 74:1637-1652, 2005.

[39] A. Drăgănescu, T. Dupont, and L. Scott. Failure of the discrete maximum principle for an elliptic finite element problem. Mathematics of Computation, 74:1-23, 2005.

[40] R. Liska and M. Shashkov. Enforcing the discrete maximum principle for linear finite element solutions for elliptic problems. Communications in Computational Physics, 3:852-877, 2008.

[41] K. B. Nakshatrala and A. J. Valocchi. Non-negative mixed finite element formulations for a tensorial diffusion equation. Journal of Computational Physics, 228:6726-6752, 2009.

[42] H. Nagarajan and K. B. Nakshatrala. Enforcing the non-negativity constraint and maximum principles for diffusion with decay on general computational grids. International Journal for Numerical Methods in Fluids, 67:820-847, 2011.

[43] K. B. Nakshatrala, H. Nagarajan, and M. Shabouei. A numerical methodology for enforcing maximum principles and the non-negative constraint for transient diffusion equations. Available on arXiv: 1206.0701, 2012.

[44] M. Kucharik, M. Shashkov, and B. Wendroff. An efficient linearity-and-bound-preserving remapping method. Journal of Computational Physics, 188:462-471, 2003.

[45] R. Garimella, M. Kucharik, and M. Shashkov. An efficient linearity and bound preserving conservative interpolation (remapping) on polyhedral meshes. Computers \&f fluids, 36:224-237, 2007.

[46] O. Burdakov, I. Kapyrin, and Y. Vassilevski. Monotonicity recovering and accuracy preserving optimization methods for postprocessing finite element solutions. Journal of Computational Physics, 231:3126-3142, 2012.

[47] C. Kreuzer. A note on why enforcing discrete maximum principles by a simple a posteriori cutoff is a good idea. arXiv:1208.3958, 2012.

[48] C. Lu, W. Huang, and E. S. V. Vleck. The cutoff method for the numerical computation of nonnegative solutions of parabolic PDEs with application to anisotropic diffusion and lubrication-type equations. Journal of Computational Physics, 242:24-36, 2013.

[49] S. Wang, G. Yuan, Y. Li, and Z. Sheng. Discrete maximum principle based on repair technique for diamond type scheme of diffusion problems. International Journal for Numerical Methods in Fluids, 70:1188-1205, 2012.

[50] X. Zhao, Y. Chen, Y. Gao, C. Yu, and Y. Li. Finite volume element methods for nonequilibrium radiation diffusion equations. International Journal for Numerical Methods in Fluids, DOI: 10.1002/fld.3838, 2013. 
[51] L. C. Evans. Partial Differential Equations. American Mathematical Society, Providence, Rhode Island, USA, 1998.

[52] D. Gilbarg and N. S. Trudinger. Elliptic Partial Differential Equations of Second Order. Springer, New York, USA, 2001.

[53] P. Pucci and J. Serrin. The Maximum Principle. Birkhäuser Verlag, Basel, Switzerland, 2007.

[54] J. Karátson and S. Korotov. Discrete maximum principles for finite element solutions of nonlinear elliptic problems with mixed boundary conditions. Numerische Mathematik, 99:669-698, 2005.

[55] M. Borsuk and V. Kondratiev. Elliptic Boundary Value Problems of Second Order in Piecewise Smooth Domains. Elsevier Science, San Diego, USA, 2006.

[56] M. E. Gurtin. An Introduction to Continuum Mechanics. Academic Press, San Diego, USA, 1981.

[57] K. Ishihara. Strong and weak discrete maximum principles for matrices associated with elliptic problems. Linear Algebra and its Applications, 88-89:431-448, 1987.

[58] M. E. Mincsovics and T. L. Hórvath. On the differences of the discrete weak and strong maximum principles for elliptic operators. In I. Lirkov, S. Margenov, and J. Waśniewski, editors, Large-Scale Scientific Computing, volume 7116 of Lecture Notes in Computer Science, pages 614-621, Berlin, Heidelberg, 2012. Springer-Verlag.

[59] A. Berman and R. J. Plemmons. Nonnegative Matrices in the Mathematical Sciences. Academic Press, New York, USA, 1979.

[60] R. S. Varga. Matrix Iterative Analysis, volume 27 of Springer Series in Computational Mathematics. SpringerVerlag, Berlin, Heidelberg, second revised and expanded edition, 2009.

[61] P.-L. George and S. Frey. Mesh Generation, volume 32. John Wiley \& Sons, Inc., New Jersey, USA, second edition, 2010.

[62] I. Babuška, J. R. Whiteman, and T. Strouboulis. Finite Elements: An Introduction to the Method and Error Estimation. Oxford University Press, New York, USA, 2011.

[63] T. Plewa, T. J. Linde, and V. G. Weirs, editors. Adaptive Mesh Refinement - Theory and Applications, volume 41 of Lecture Notes in Computational Science and Engineering. Springer, Berlin, Germany, 2005.

[64] G. S. Payette, K. B. Nakshatrala, and J. N. Reddy. On the performance of high-order finite elements with respect to maximum principles and the non-negative constraint for diffusion-type equations. International Journal for Numerical Methods in Engineering, 91:742-771, 2012.

[65] T. Vejchodský. Angle conditions for discrete maximum principles in higher-order FEM, pages 901-909. Numerical Mathematics and Advanced Applications 2009. Springer-Verlag, 2010.

[66] A. Prestel and C. N. Delzell. Positive Polynomials: From Hilbert's 17th Problem to Real Algebra. Springer Monographs in Mathematics. Springer-Verlag, Berlin, Heidelberg, Germany, 2001.

[67] B. Reznick. Some concrete aspects of Hilbert's 17th problem. Contemporary Mathematics, 253:251-272, 2000.

[68] P. G. Ciarlet. Discrete variational Green's function - I. Aequationes Mathematicae, 4:74-82, 1970.

[69] P. G. Ciarlet and R. S. Varga. Discrete variational Green's function - II. Numerische Mathematik, 16:115-128, 1970 .

[70] D. W. Höhn and D. H. D. Mittelmann. Some remarks on the discrete maximum-principle for finite elements in higher order. Computing, 27:145-154, 1981.

[71] P. Šolín and T. Vejchodský. A weak discrete maximum principle for hp-FEM. Journal of Computational and Applied Mathematics, 209:54-65, 2007.

[72] W. Huang. Metric tensors for anisotropic mesh generation. Journal of Computational Physics, 204:633-665, 2005.

[73] R. Schneider. A review of anisotropic refinement methods for triangular meshes in FEM. In T. Apel and O. Steinbach, editors, Advanced Finite Element Methods and Applications, volume 66 of Lecture Notes in Applied and Computational Mechanics, pages 133-152, Berlin, Heidelberg, 2013. Springer-Verlag.

[74] M. Křížek and L. Qun. On diagonal dominance of stiffness matrices in 3D. East-West Journal of Numerical Mathematics, 3:59-69, 1995.

[75] K. Lipnikov, M. Shashkov, D. Svyatskiy, and Y. Vassilevski. Monotone finite volume schemes for diffusion equations on unstructured triangular and shape-regular polygonal meshes. Journal of Computational Physics, 227:492-512, 2007.

[76] Gmsh: A three-dimensional finite element mesh generator with pre- and post-processing facilities. URL: http://www.geuz.org/gmsh/. 
[77] J. H. Brandts, S. Korotov, and M. Kř́̌žek. The discrete maximum principle for linear simplicial finite element approximations of a reaction-diffusion problem. Linear Algebra and its Applications, 429:2344-2357, 2008.

[78] A. J. Wathen. An analysis of some element-by-element techniques. Computer Methods in Applied Mechanics and Engineering, 74:271-287, 1989.

[79] M. T. Heath. Scientific Computing-An Introductory Survey. McGraw-Hill, New York, USA, second edition, 2005.

[80] S. Wolfram. Mathematica, Version 9.0.1. Wolfram Research, Inc., Champaign, Illinois, USA, 2013.

[81] M. J. Castro-Diaz, F. Hecht, B. Mohammadi, and O. Pironneau. Anisotropic unstructured mesh adaption for flow simulations. International Journal for Numerical Methods in Fluids, 25:475-491, 1997.

[82] W. Huang and X. Li. An anisotropic mesh adaptation method for the finite element solution of variational problems. Finite Elements in Analysis and Design, 46:61-73, 2010.

[83] P. J. Frey and F. Alauzet. Anisotropic mesh adaptation for CFD computations. Computer Methods in Applied Mechanics and Engineering, 194:5068-5082, 2005.

[84] R. Kornhuber and R. Roitzsch. On adaptive grid refinement in the presence of internal or boundary layers. IMPACT of Computing in Science and Engineering, 2:40-72, 1990.

[85] S. Yamakawa and K. Shimada. Anisotropic tetrahedral meshing via bubble packing and advancing front. International Journal for Numerical Methods in Engineering, 57:1923-1942, 2003.

[86] W. Rachowicz. An anisotropic h-type mesh-refinement strategy. Computer Methods in Applied Mechanics and Engineering, 109:169-181, 1993.

[87] F. J. Bossen and P. S. Heckbert. A pliant method for anisotropic mesh generation. In 5th International Meshing Roundtable, Sandia National Laboratory, pages 63-74, 1996.

[88] W. Huang. Variational mesh adaptation: Isotropy and equidistribution. Journal of Computational Physics, 174:903-924, 2001.

[89] M. J. Cloud and B. C. Drachman, editors. Inequalities: With Applications to Engineering. Springer-Verlag, New York, USA, 1998.

[90] CGAL: Computational Geometry Algorithms Library. http://www.cgal.org.

[91] Simmetrix-MeshSim, Automatic Mesh Generation Suite. Simmetrix, Inc., Website: http://www.simmetrix.com/, Clifton Park, New York, USA.

[92] H. Nguyen, M. Gunzburger, L. Ju, and J. Burkardt. Adaptive anisotropic meshing for steady convectiondominated problems. Computer Methods in Applied Mechanics and Engineering, 198:2964-2981, 2009.

[93] Y. Q. Huang, Y. F. Su, H. Y. Wei, and N. Y. Yi. Anisotropic mesh generation methods based on ACVT and natural metric for anisotropic elliptic equation. Science China Mathematics, 56:2615-2630, 2013.

[94] J. N. Reddy. Finite Element Method. McGraw Hill, New York, USA, 1993.

[95] S. Boyd and L. Vandenberghe. Convex Optimization. Cambridge University Press, Cambridge, UK, 2004.

[96] J. Donea and A. Huerta. Finite Element Methods for Flow Problems. John Wiley \& Sons, Inc., Chichester, UK, 2003.

[97] R. Taylor and R. Krishna. Multicomponent Mass Transfer. Wiley Series in Chemical Engineering. John Wiley \& Sons Inc., New York, USA, 1993.

[98] T. J. Chung. Computational Fluid Dynamics. Cambridge University Press, New York, USA, second edition, 2010.

[99] R. Therrien and E. A. Sudicky. Three-dimensional analysis of variably-saturated flow and solute transport in discretely-fractured porous media. Journal of Contaminant Hydrology, 23:1-44, 1996.

[100] G. F. Pinder and M. A. Celia. Subsurface Hydrology. John Wiley \& Sons, Inc., New Jersey, USA, 2006.

[101] C. Zheng and G. D. Bennett. Applied Contaminant Transport Modeling. John Wiley \& Sons, Inc., New York, USA, second edition, 2002.

[102] P. L. McCarty and C. S. Criddle. Chemical and biological processes: The need for mixing. In P. K. Kitanidis and P. L. McCarty, editors, Delivery and Mixing in the Subsurface: Processes and Design Principles for In Situ Remediation, pages 7-52. Springer, 2012.

[103] M. Dentz, T. Le Borgne, A. Englert, and B. Bijeljic. Mixing, spreading and reaction in heterogeneous media: A brief review. Journal of Contaminant Hydrology, 120-121:1-17, 2011.

[104] B. A. Zinn and L. F. Konikow. Effects of intraborehole flow on groundwater age distribution. Hydrogeology Journal, 15:633-643, 2007. 


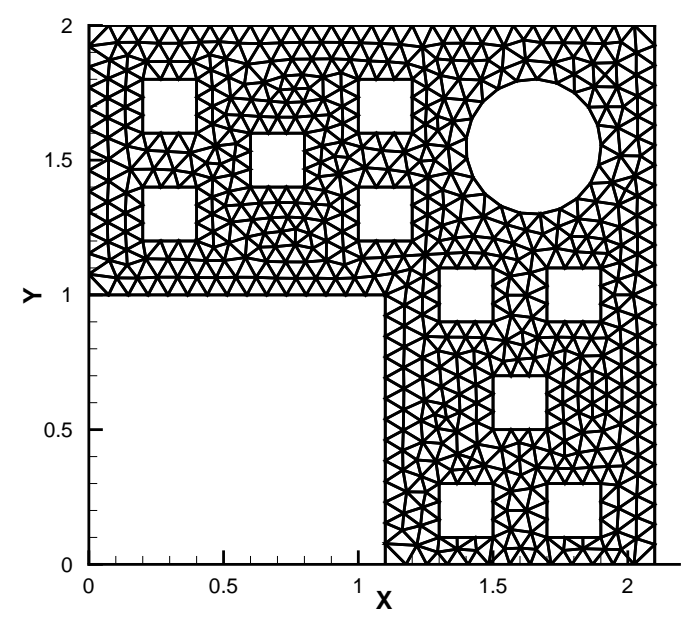

(a) T3 mesh

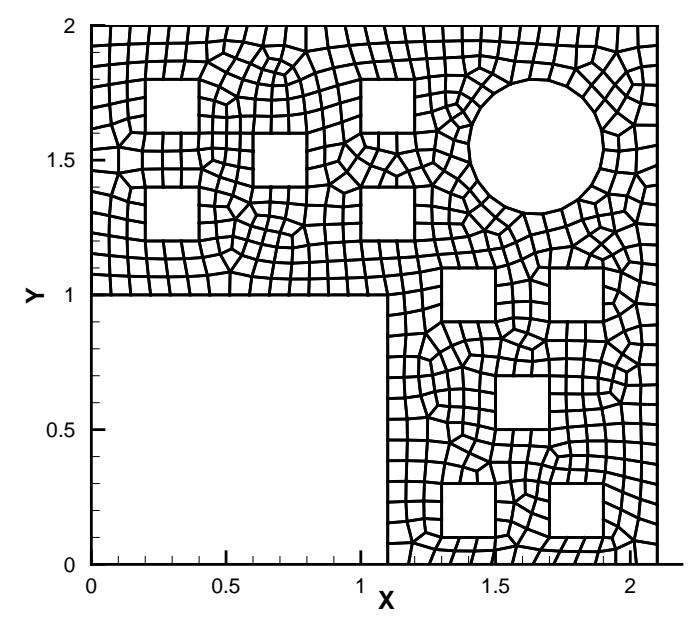

(b) Q4 mesh

Figure 1. ABAQUS UNSTRUCTURED MESHES FOR AN L-SHAPED DOMAIN WITH MULTIPLE HOLES: The left and right figures show an instance of three-node triangular and four-node quadrilateral meshes employed in the numerical simulation of a pure anisotropic diffusion problem using ABAQUS.

[105] L. F. Konikow and G. Z. Hornberger. Modeling effects of multinode wells on solute transport. Groundwater, 44:648-660, 2006.

[106] A. Mejía, E. Cassiraga, and A. Sahuquillo. Influence of hydraulic conductivity and wellbore design in the fate and transport of nitrate in multi-aquifer systems. Mathematical Geosciences, 44:227-238, 2012.

[107] T. Myers. Potential contaminant pathways from hydraulically fractured shale to aquifers. Groundwater, 50:872882, 2012.

[108] J. E. Saiers and E. Barth. Potential contaminant pathways from hydraulically fractured shale to aquifers. Groundwater, 50:826-828, 2012.

[109] H. A. Cohen, T. Parratt, and C. B. Andrews. Potential contaminant pathways from hydraulically fractured shale to aquifers. Groundwater, 51:317-319, 2013.

[110] C. B. Avci. Evaluation of flow leakage through abandoned wells and boreholes. Water Resources Research, 30:2565-2578, 1994.

[111] S. Lacombe, E. A. Sudicky, S. K. Frape, and A. J. A. Unger. Influence of leaky boreholes on cross-formational groundwater flow and contaminant transport. Water Resources Research, 31:1871-1882, 1995.

[112] A. Ebigbo, H. Class, and R. Helmig. $\mathrm{CO}_{2}$ leakage through an abandoned well: Problem-oriented benchmarks. Computational Geosciences, 11:103-115, 2007.

[113] K. B. Nakshatrala and D. Z. Turner. A mixed formulation for a modification to Darcy equation based on Picard linearization and numerical solutions to large-scale realistic problems. International Journal for Computational Methods in Engineering Science and Mechanics, 14:524-541, 2013.

[114] T. J. R. Hughes, G. Engel, L. Mazzei, and M. G. Larson. The continuous Galerkin method is locally conservative. Journal of Computational Physics, 163:467-488, 2000.

[115] R. D. Cook, D. S. Malkus, M. E. Plesha, and R. J. Witt. Concepts and Applications of Finite Element Analysis. John Wiley \& Sons, Inc., New York, USA, fourth edition, 2002.

[116] O. C. Zienkiewicz, R. L. Taylor, and J. Z. Zhu. The Finite Element Method: Its Basis and Fundamentals. Butterworth-Heinemann, Elsevier Ltd., Oxford, UK, seventh edition, 2013.

[117] W. Huang and Y. Wang. Discrete maximum principle for the weak Galerkin method for anisotropic diffusion problems. arXiv:1401.6232, 2014. 


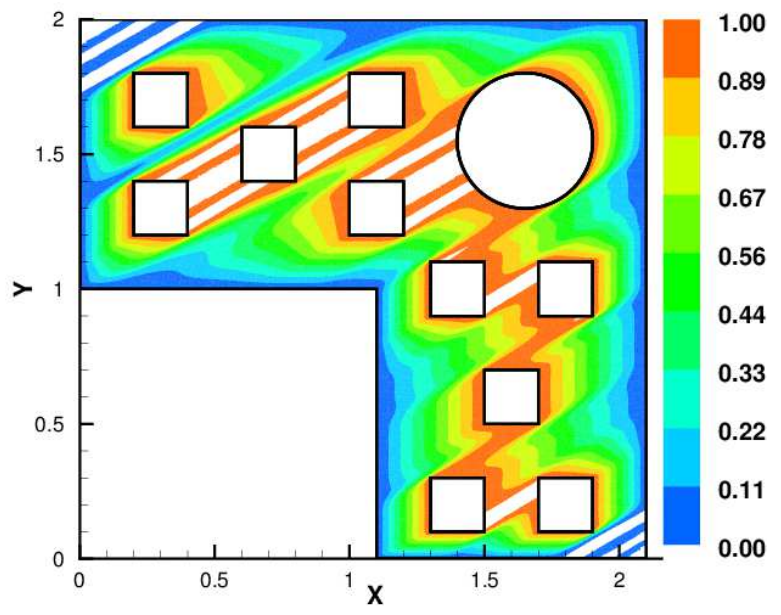

Figure 2. ABAQUS NUMERICAL SIMULATION FOR AN L-SHAPED DOMAIN WITH MULTIPLE HOLES: The contours of concentration obtained using ABAQUS are based on three-node triangular mesh.

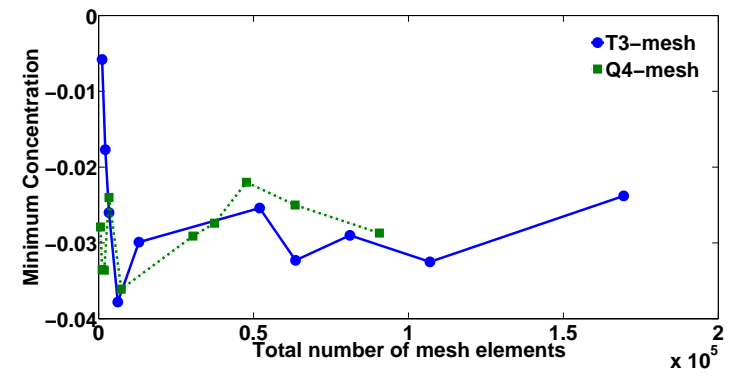

(a) Non-negative constraint violation

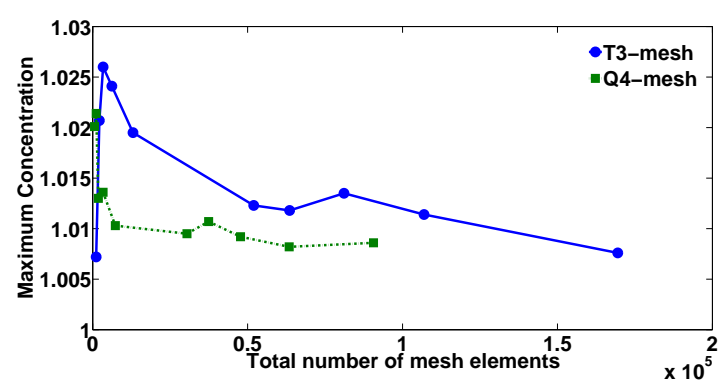

(b) Maximum constraint violation

Figure 3. Minimum And MAXimum VAlues For CONCENTRATion in AN L-SHAPED DOMAIN WITH MULTIPLE HOLES: The left and right figures show the minimum and maximum values attained in the computational domain based on the numerical simulations for various three-node triangular and four-node quadrilateral meshes using ABAQUS. From the above figures, it is evident that the violation in the non-negative and maximum constraints do not reduce on mesh refinement. 


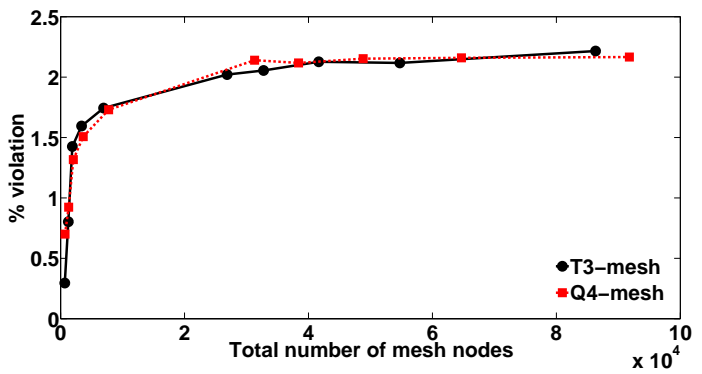

(a) \% violation: Non-negative constraint

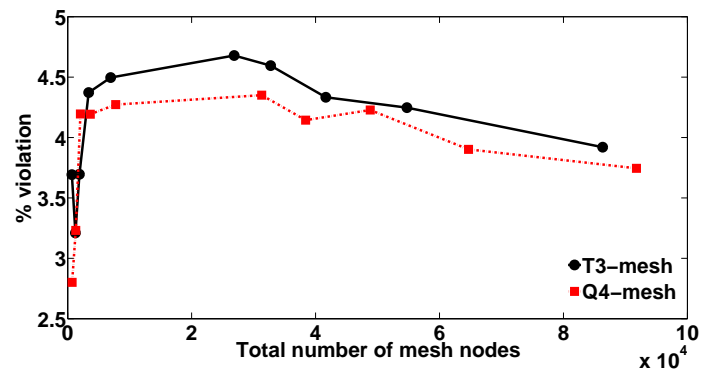

(b) \% violation: Maximum constraint

Figure 4. Percentage of Violation in minimum and maximum Constraints For CONCENTRATION IN AN L-SHAPED DOMAIN WITH MULTIPlE HOLEs: The left and right figures show the percentage of nodes that have violated the non-negative and maximum constraint for concentration obtained using ABAQUS. These numerical results are based on various three-node triangular and four-node quadrilateral meshes. In both of these cases, the percentage of mesh nodes that violated these constraints never decreases to zero.

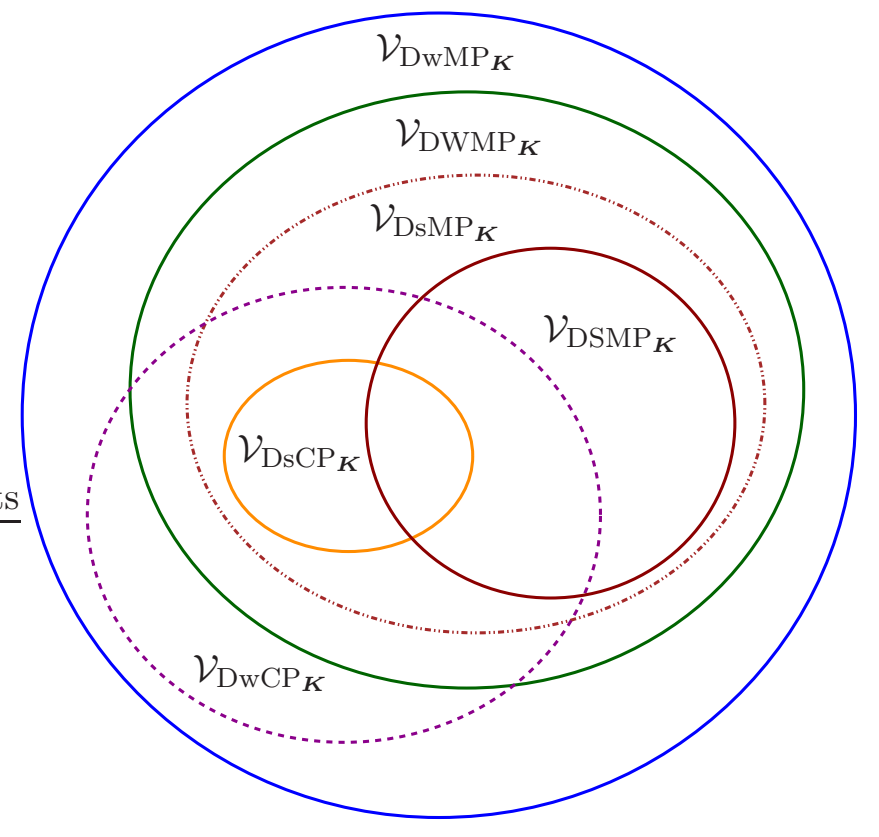

Figure 5. VEnN DiAGRAM FOR THE SPACE OF SOLUTIONS BASED ON MESH RESTRICTIONS: A pictorial description of the space of numerical solutions satisfying various DMPs and DCPs based on equation (2.25) and Theorem 2.6. It is evident from the above figure that $\mathcal{V}_{\mathrm{DSMP}_{K}} \subset \mathcal{V}_{\mathrm{DsMP}_{K}} \subset \mathcal{V}_{\mathrm{DWMP}_{K}} \subset \mathcal{V}_{\mathrm{DwMP}_{K}}$ and $\mathcal{V}_{\mathrm{DsCP}_{K}} \subset \mathcal{V}_{\mathrm{DwCP}_{K}}$. But we would like to emphasize that we do not have the following enclosures: $\mathcal{V}_{\mathrm{DwCP}_{K}} \subset \mathcal{V}_{\mathrm{DWMP}_{K}}$ and $\mathcal{V}_{\mathrm{DsCP}_{K}} \subset \mathcal{V}_{\mathrm{DSMP}_{K}}$. 


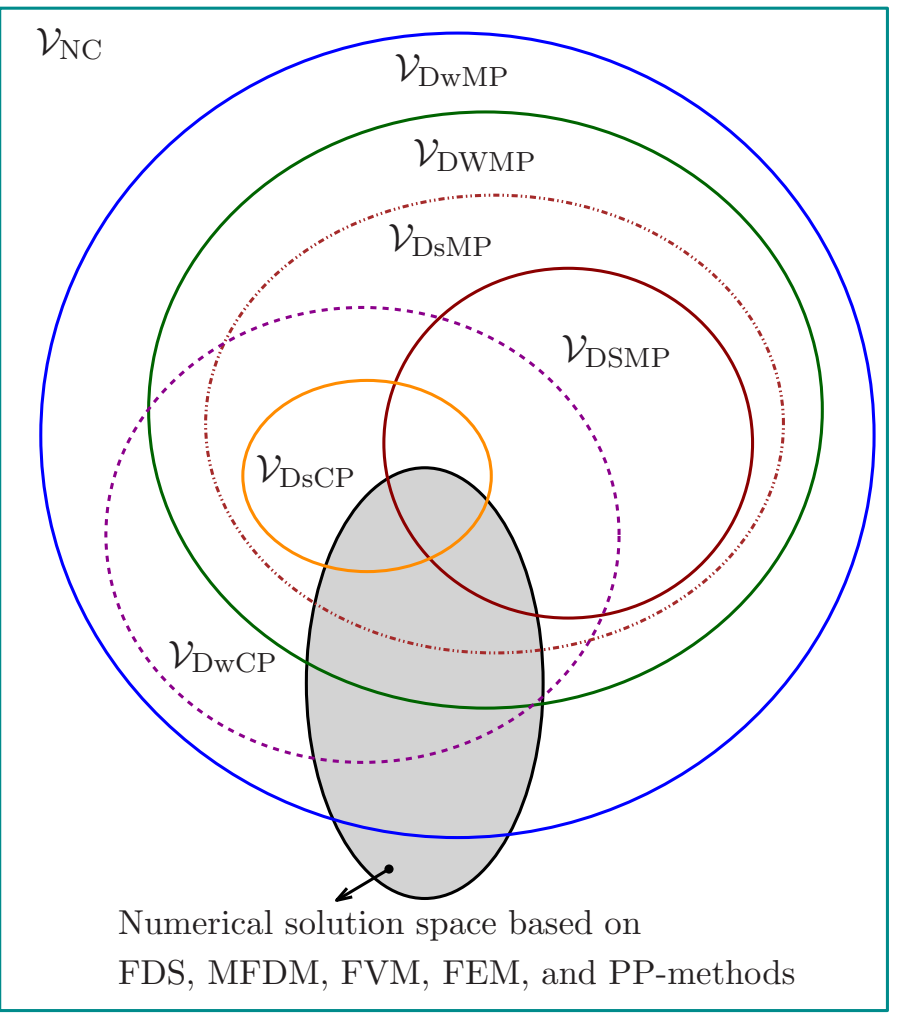

Figure 6. VENN DIAGRAM FOR THE SPACE OF SOlUtions BASED ON VARIOUS NUMERICAL FORMULATIONS: A pictorial description of the space of numerical solutions satisfying various DMPs, DCPs, and NC. In numerical literature, most of the numerical methods that exist to satisfy various DMPs are mainly non-linear. In the past decade, considerable advancements have been made to fulfill various version of DMPs for a certain class of linear elliptic and parabolic partial differential equations. But it should be noted that there is seldom research progress related to satisfaction of different DCPs (see [11, Section 4]). Hence, we would like to highlight that developing a general and variationally consistent numerical technique to encompass all these discrete principles is still an open problem. 

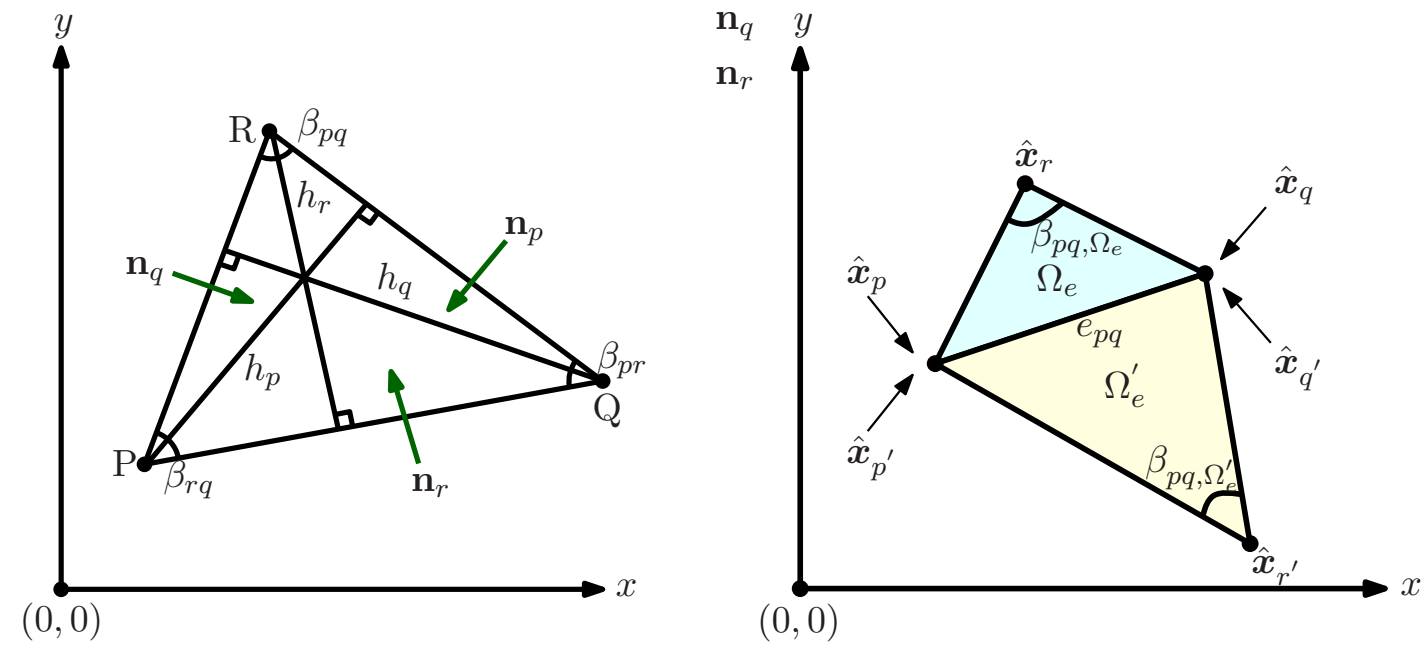

Figure 7. Geometrical properties of an arbitrary simplex in 2D: The left figure shows a pictorial description of various geometrical properties, such as unit inward normals $\left(\mathbf{n}_{p}, \mathbf{n}_{q}\right.$, and $\left.\mathbf{n}_{r}\right)$, dihedral angles in Euclidean metric $\left(\beta_{p q}, \beta_{p r}\right.$, and $\left.\beta_{r q}\right)$, and heights $\left(h_{p}\right.$, $h_{q}$, and $h_{r}$ ) of an arbitrary element $\Omega_{e} \in \mathcal{T}_{h}$. Correspondingly, the vertices of this triangle PQR are given by $\hat{\boldsymbol{x}}_{p}, \hat{\boldsymbol{x}}_{q}$, and $\hat{\boldsymbol{x}}_{r}$. The right figure shows an arbitrary patch of elements $\Omega_{e}$ and $\Omega_{e}^{\prime}$, (which belong to the triangulation $\mathcal{T}_{h}$ ) sharing a common edge $e_{p q}$. The edge $e_{p q}$ connects the coordinates $\hat{\boldsymbol{x}}_{p}\left(=\hat{\boldsymbol{x}}_{p^{\prime}}\right)$ and $\hat{\boldsymbol{x}}_{q}\left(=\hat{\boldsymbol{x}}_{q^{\prime}}\right)$. The dihedral angles in Euclidean metric opposite to edge $e_{p q}$ are denoted by $\beta_{p q, \Omega_{e}}$ and $\beta_{p q, \Omega_{e}^{\prime}}$.
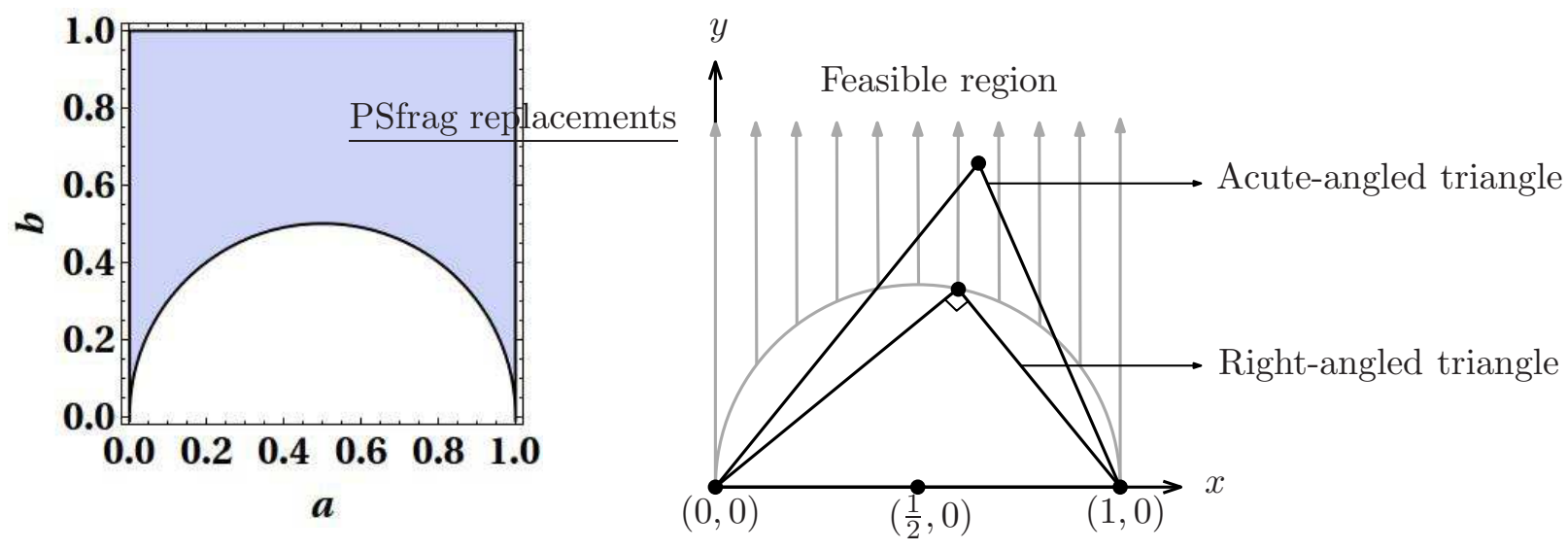

Figure 8. T3 element for heterogeneous isotropic Diffusivity: A pictorial description of the feasible region (left figure) is shown in light blue color. The right figure indicates that the point $(a, b)$ can lie either on the circle with center $\left(\frac{1}{2}, 0\right)$ and radius $\frac{1}{2}$ or outside the circular region. The points within the circular region are infeasible. This results in two possibilities for choosing a T3 element in the realm of the feasible region, which is either a right-angled triangle or an acute-angled triangle. 


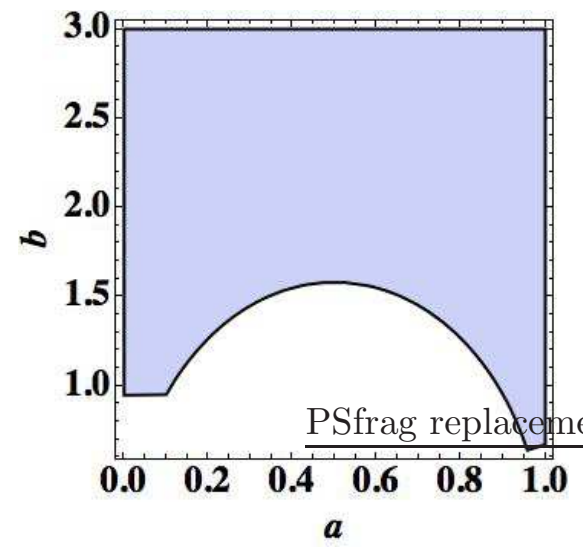

(a) Feasible region for $(a, b)$

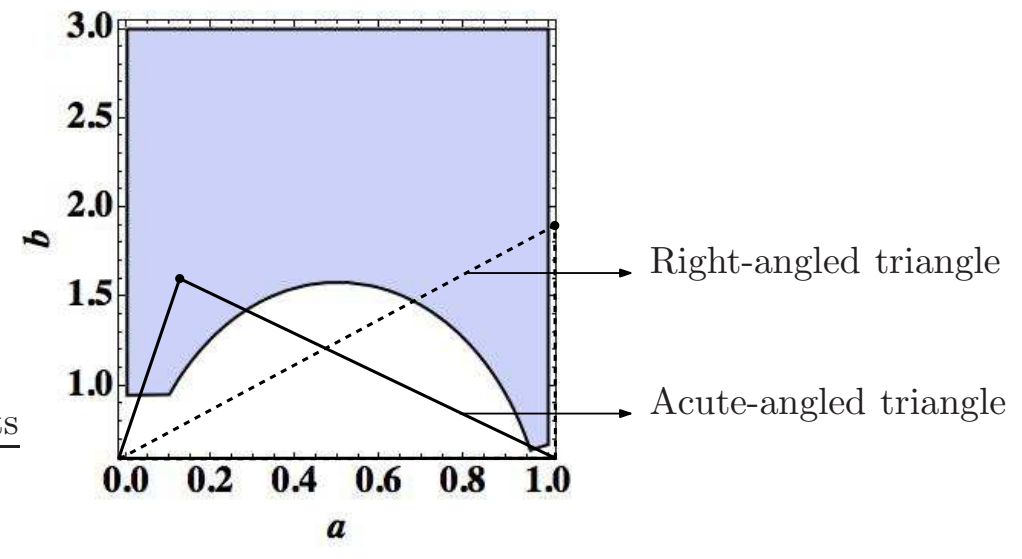

(b) Possible T3 elements when $\widetilde{D}_{x y}=0$

Figure 9. T3 ELEMENT For Anisotropic Diffusivity when $\widetilde{D}_{x y}=0$ : A pictorial description of the feasible region (left figure) for the coordinates $(a, b)$ is indicated in light blue color. The numerical values for the two parameters, which decide the feasible region, are chosen to be $\epsilon=10$ and $\eta=0$. In this case, the right figure indicates that acute-angled and right-angled triangles are possible. As $\epsilon$ increases, the coordinate $b$ has to increase proportionally to satisfy the inequality given by the equation (4.33a).

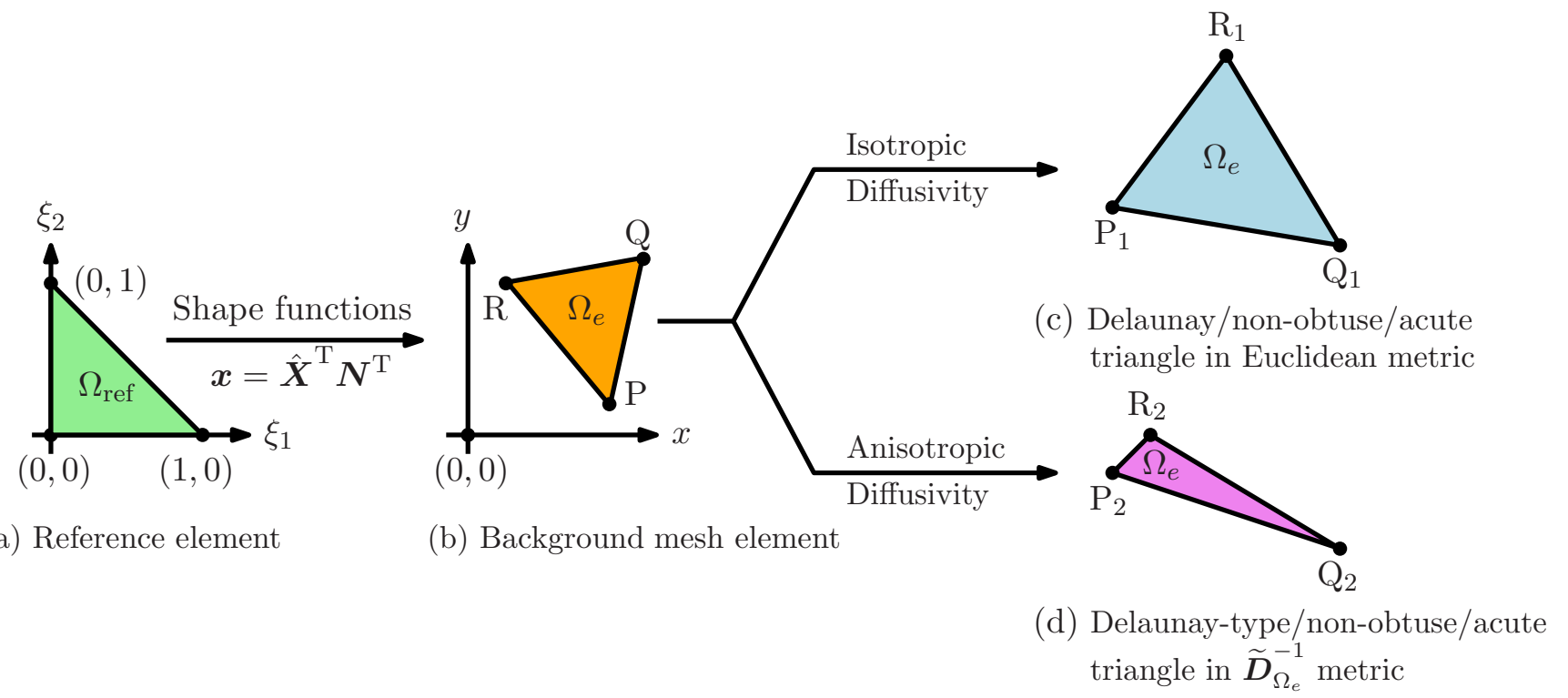

Figure 10. DMP-Based T3 elements For heterogeneous isotropic AND ANISOTROPIC DIFFUSIVITY: A pictorial description of a mesh generation procedure to obtain a new triangulation using a given background mesh. This new simplicial mesh satisfies various discrete properties as contrary to the background mesh. The procedure to obtain such a triangulation is iterative and is based on Theorems 4.3 and 4.4. 


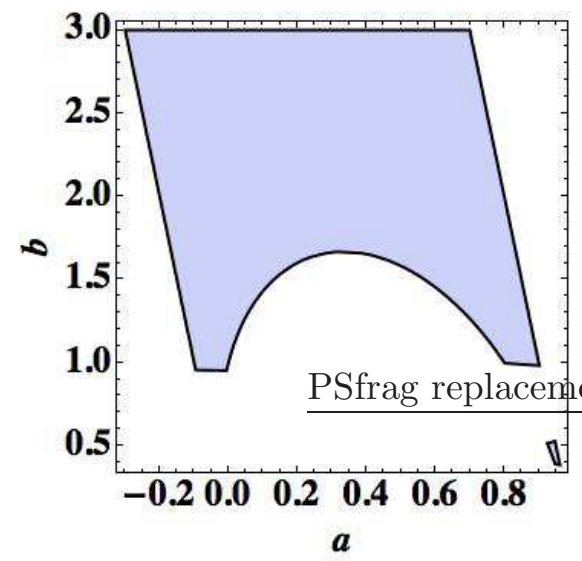

(a) Feasible region for $(a, b)$

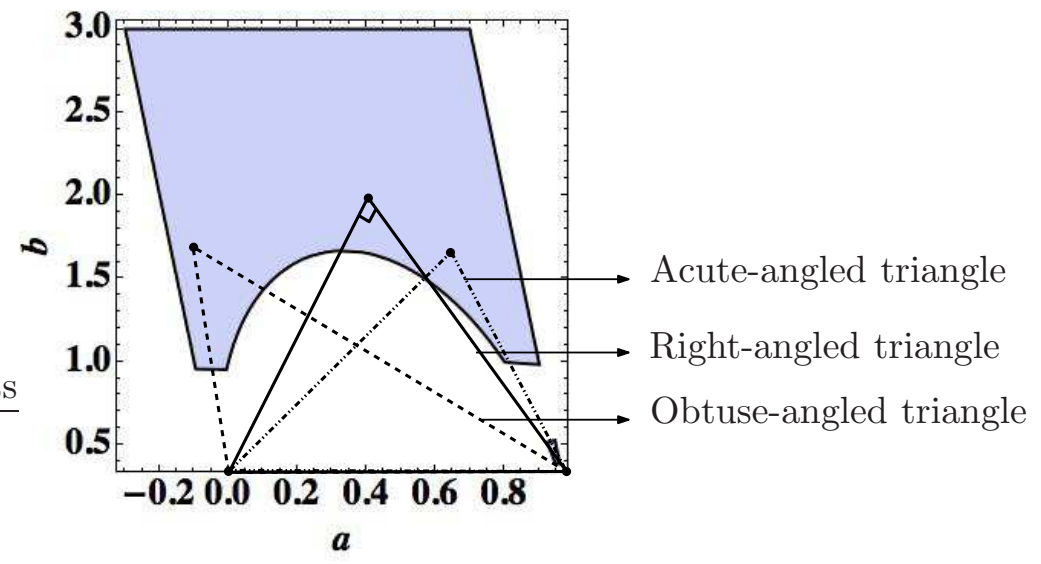

(b) Possible T3 elements when $\widetilde{D}_{x y}<0$

Figure 11. T3 ELEMENT FOR ANisotropic Diffusivity when $\widetilde{D}_{x y}<0$ : The left figure indicates the feasible region for the coordinates $(a, b)$ in light blue color. The right figure indicates that when $\widetilde{D}_{x y}<0$, the (Euclidean metric) dihedral angles in the T3 element can be acute-angled or right-angled or even obtuse-angled. In this case, we have chosen $\epsilon=10$ and $\eta=-1$. For a fixed $\eta$ as $\epsilon$ increases, the value of coordinate $b$ also increases. So it is a daunting task to find a viable T3 element. One can also notice that the feasible region is not contiguous.

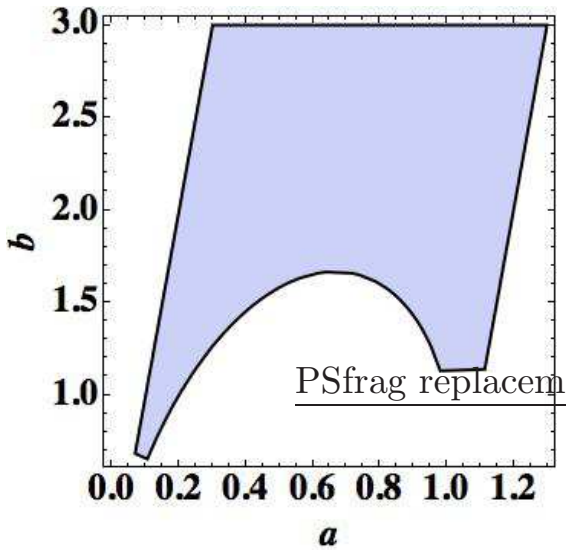

(a) Feasible region for $(a, b)$

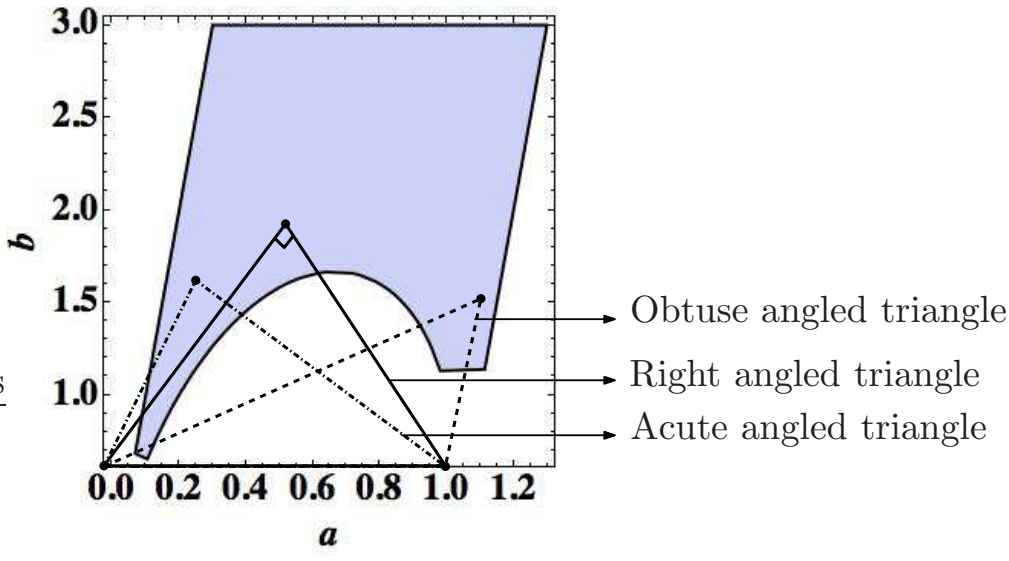

(b) Possible T3 elements when $\widetilde{D}_{x y}>0$

Figure 12. T3 ELEMENT FOR ANisotropic Diffusivity When $\widetilde{D}_{x y}>0$ : The left figure indicates the feasible region for the coordinates $(a, b)$ in light blue color. The right figure indicates that when $\widetilde{D}_{x y}>0$, the (Euclidean metric) dihedral angles in the T3 element can be acute-angled or right-angled or even obtuse-angled. In this case, we have chosen $\epsilon=10$ and $\eta=1$. For a fixed $\eta$ as $\epsilon$ increases, the value of coordinate $b$ also increases. For higher values of $\epsilon$, it is very difficult to find a suitable T3 element, which can mesh any given computational domain. 

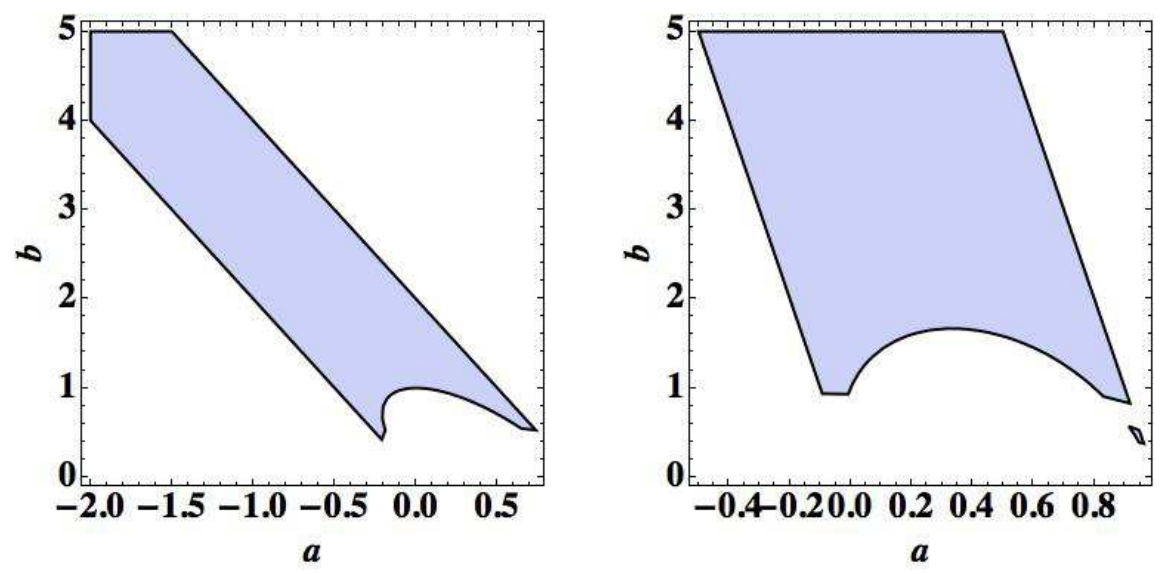

(a) $\epsilon=2$ and $\eta=-1$

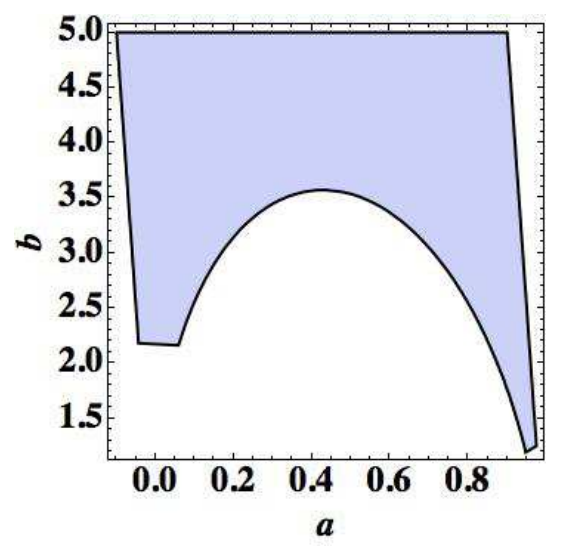

(b) $\epsilon=10$ and $\eta=-1$

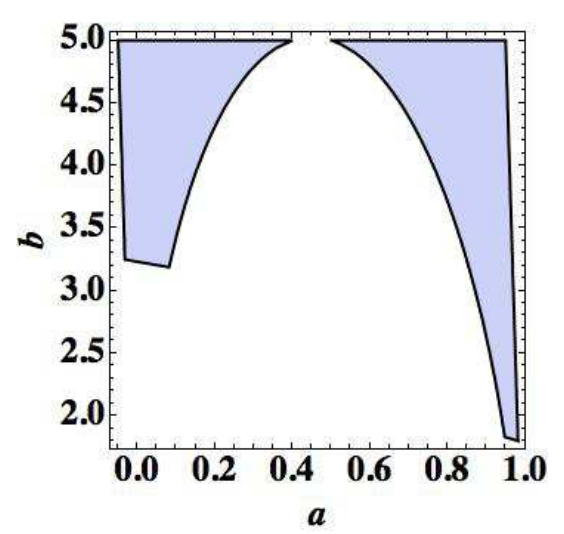

(c) $\epsilon=50$ and $\eta=-1$

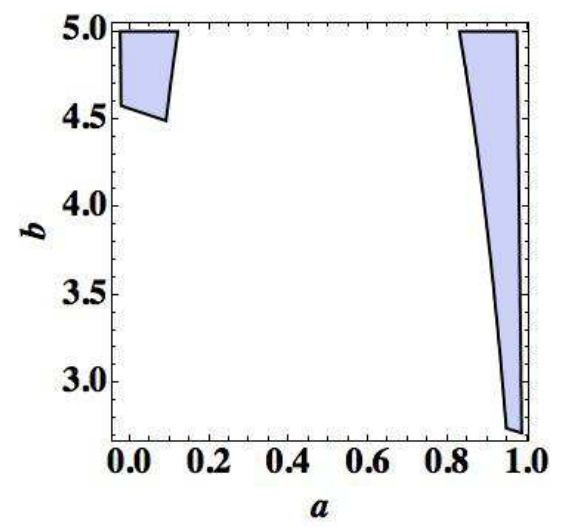

(d) $\epsilon=100$ and $\eta=-1$

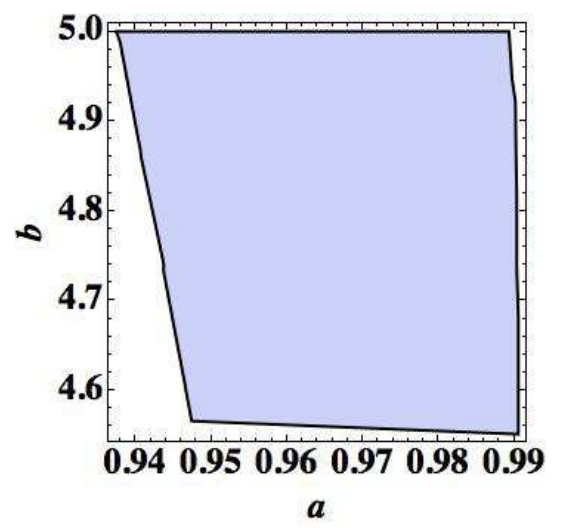

(e) $\epsilon=200$ and $\eta=-1$

(f) $\epsilon=500$ and $\eta=-1$

Figure 13. T3 ElEMENT For FIXED $\eta$ AND VARYing $\epsilon$ : A pictorial description of the feasible region (light blue color) for a fixed $\eta$ and varying $\epsilon$. Analysis is performed for $\eta=-1$ and $\epsilon=\{2,10,50,100,200,500\}$. It is evident there is a drastic variation in the feasible region as $\epsilon$ increases. 


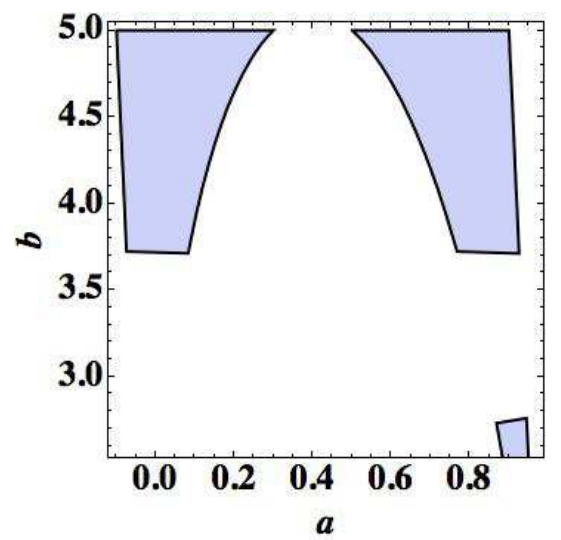

(a) $\epsilon=100$ and $\eta=-2$

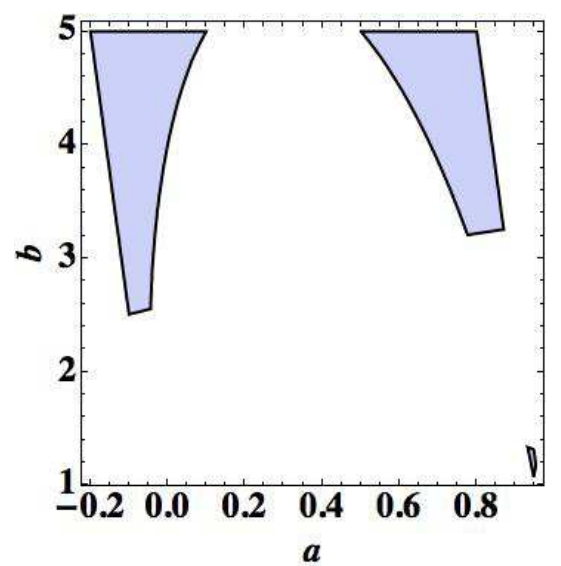

(c) $\epsilon=100$ and $\eta=-4$

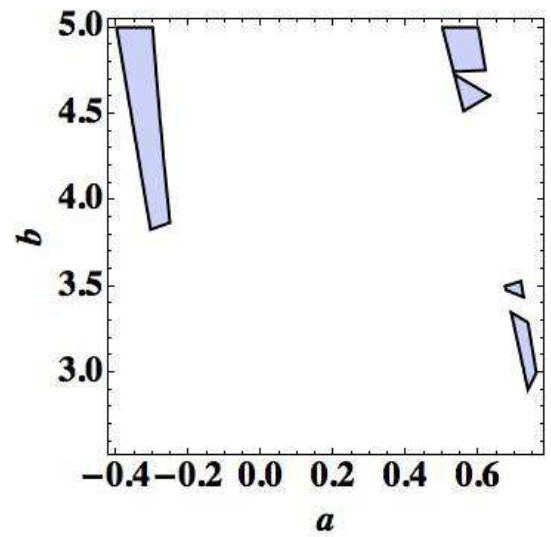

(e) $\epsilon=100$ and $\eta=-8$

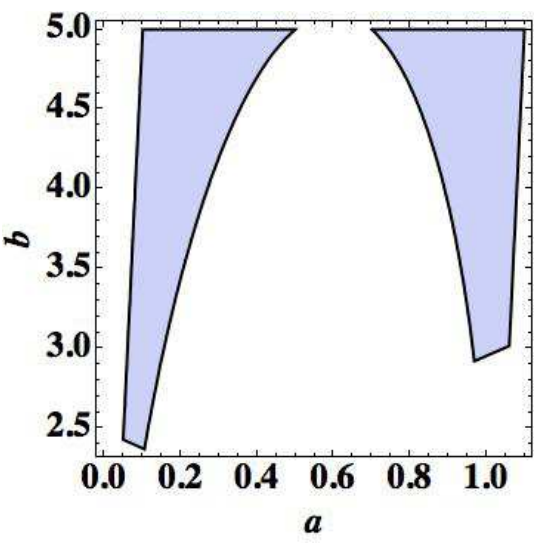

(b) $\epsilon=100$ and $\eta=2$

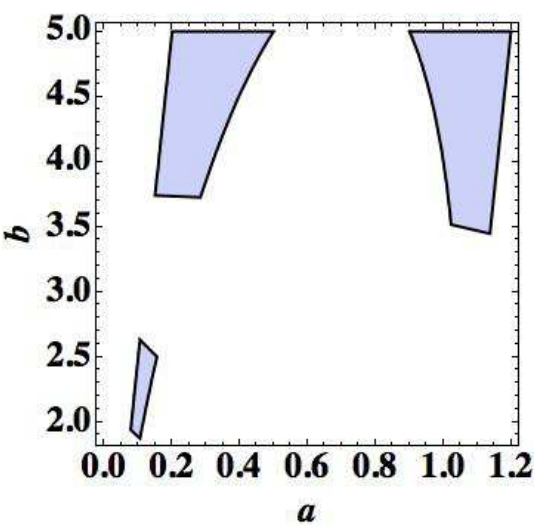

(d) $\epsilon=100$ and $\eta=4$

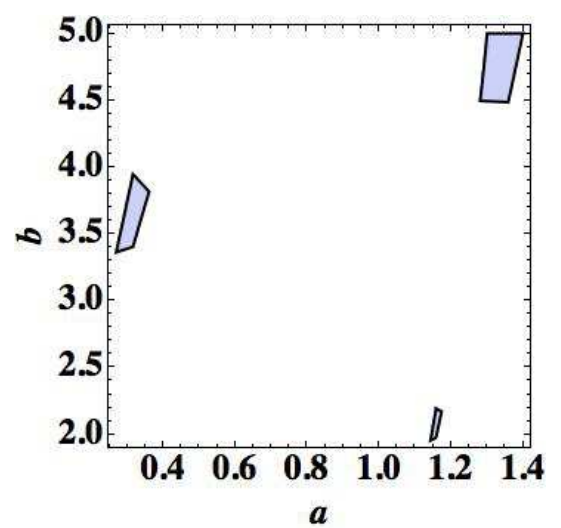

(f) $\epsilon=100$ and $\eta=8$

Figure 14. T3 ELEMENT FOR FIXED $\epsilon$ AND VARYING $\eta$ : A pictorial description of the feasible region (light blue color) for a fixed $\epsilon$ and varying $\eta$. Analysis is performed for $\epsilon=100$ and $\eta=\{-8,-4,-2,2,4,8\}$. It is evident there is considerable variation in the feasible region as $\eta$ changes. Also, there is no fixed pattern on this variation about $\eta$. 


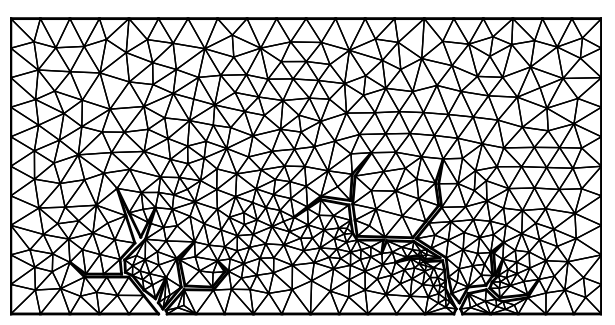

$\begin{array}{lllllllllll}0 & 0.25 & 0.5 & 0.75 & 1 & 1.25 & 1.5 & 1.75 & 2 & 2.25 & 2.5\end{array}$

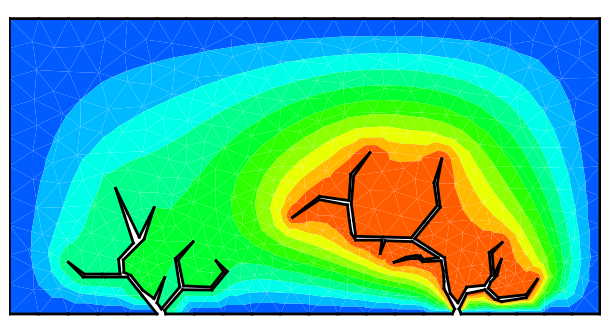

(a) Delaunay-Voronoi mesh: $N v=539$ and Nele $=906$

(b) $\mathbf{v}=(0,0)$ and $\alpha=0$
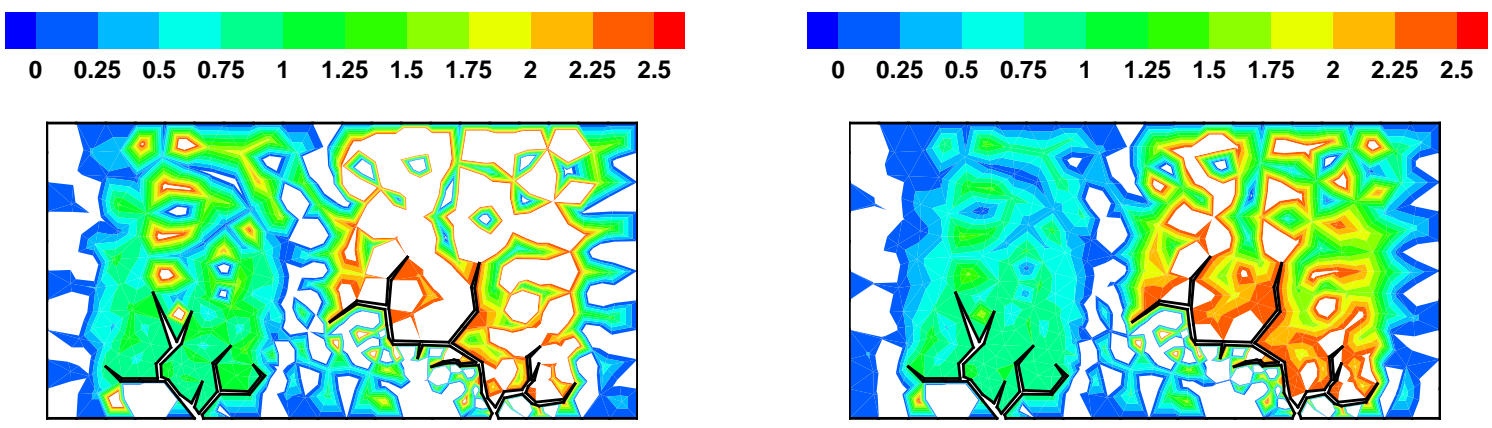

(c) $\mathbf{v}=(0.1,1.0)$ and $\alpha=0$

(d) $\mathbf{v}=(0.1,1.0)$ and $\alpha=1.0$

Figure 15. Test Problem \#1: The top left figure shows a coarse triangulation (generated using Gmsh based on Algorithm 1) employed in the numerical study, which is to the scale. The top right figure and the bottom two figures show the concentration profiles obtained for various values of the velocity field and linear reaction coefficient using this mesh. 


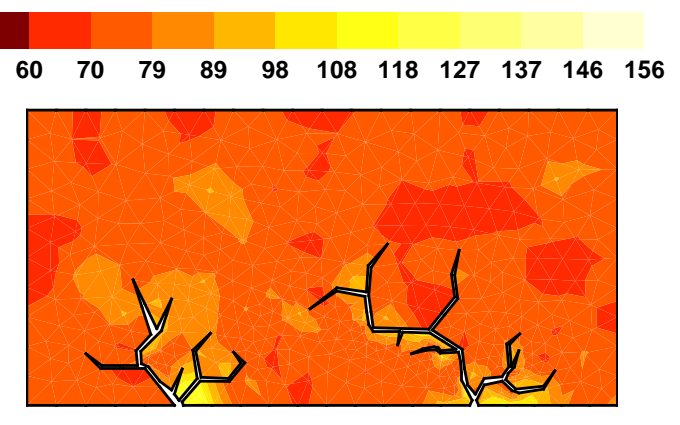

(a) Element maximum angles: $N v=539$ and $N e l e=906$

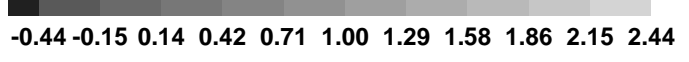

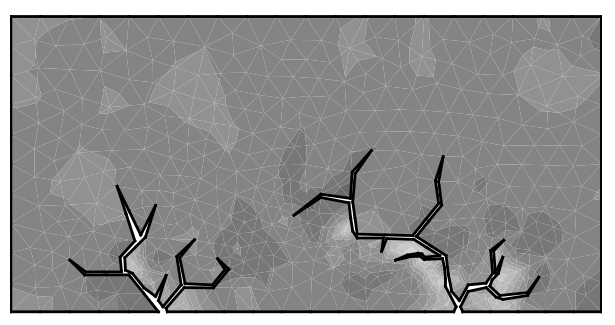

(c) Delaunay-type condition: $\mathbf{v}=(0.1,1.0)$ and $\alpha=0$

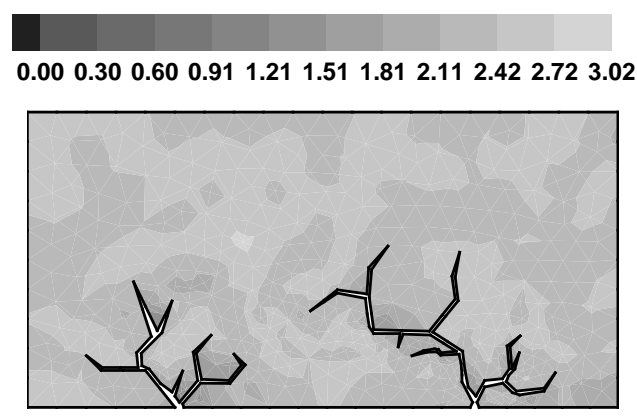

(b) Delaunay-type condition: $\mathbf{v}=(0,0)$ and $\alpha=0$
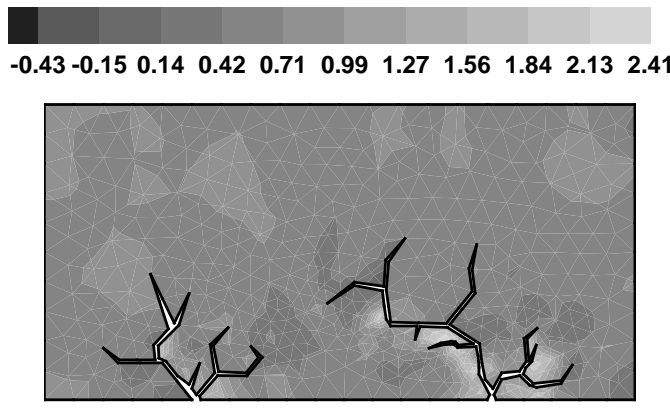

Figure 16. TeSt problem \#1: The top left figure shows the maximum angle possible in each element of the mesh. The top right figure and the bottom two figures show the element maximum generalized Delaunay-type condition, which is a weaker condition as compared to the element maximum anisotropic non-obtuse angle condition. 


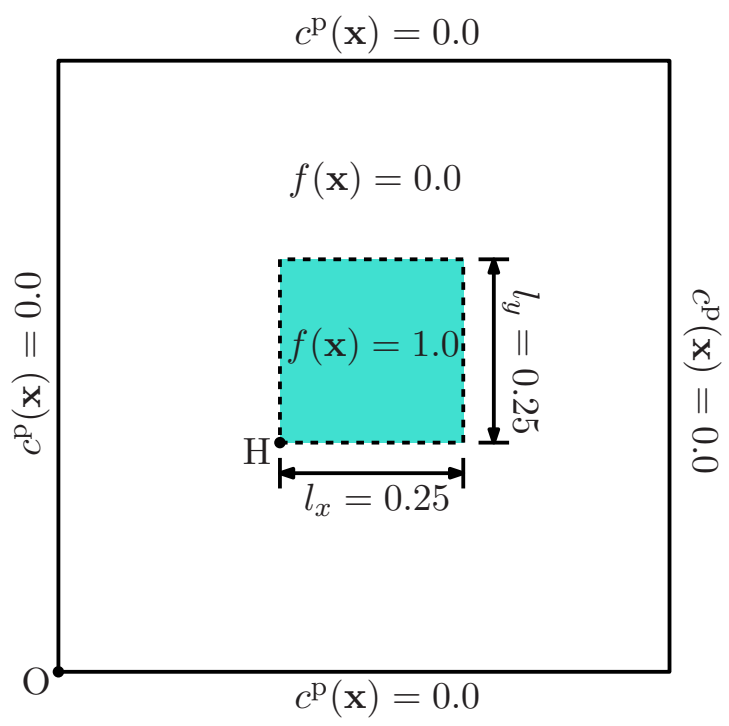

Figure 17. Test problem \#2: The computational domain under consideration is a biunit square with one of its vertices at origin $\mathrm{O}=(0,0)$. Homogeneous Dirichlet boundary conditions are prescribed on all sides of the square. The volumetric source $f(\mathbf{x})$ is zero inside the domain, except for the square region (including the boundaries) located at vertex $\mathrm{H}=(0.375,0.375)$. In this region, $f(\mathbf{x})$ is equal to unity. Herein, we assume that the velocity vector field and linear reaction coefficient are equal to zero everywhere in the computational domain.
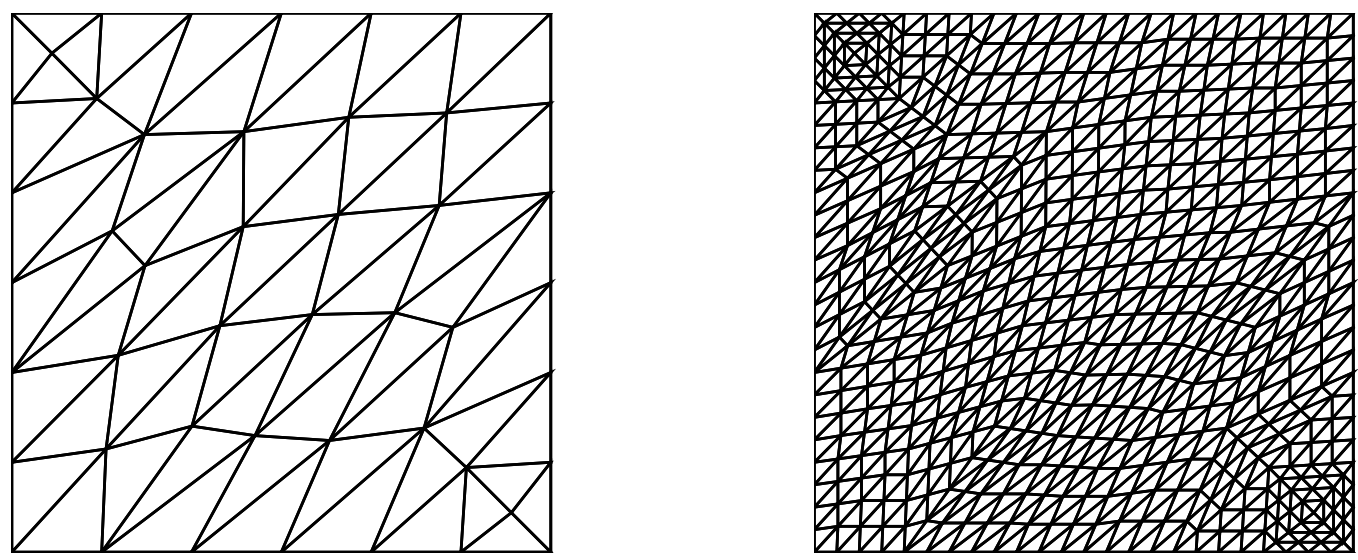

(a) Background mesh: $N v=47$ and $N e l e=68$

(b) Anisotropic mesh: $N v=593$ and $N e l e=1088$

Figure 18. Test Problem \#2: The left figure shows the background mesh on which BAMG operates to give an anisotropic triangulation, which is shown in the right figure. As the ratio of the minimum eigenvalue of anisotropic diffusivity tensor to its maximum is 0.1 , which is not very high, so the resulting triangulation consists of a mixture of skinny and normal triangles. 


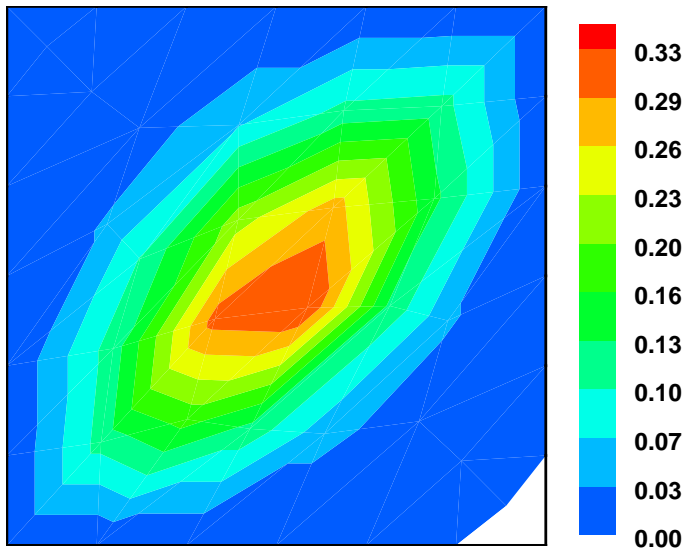

(a) Background mesh: $\mathbf{v}=(0,0)$ and $\alpha=0$

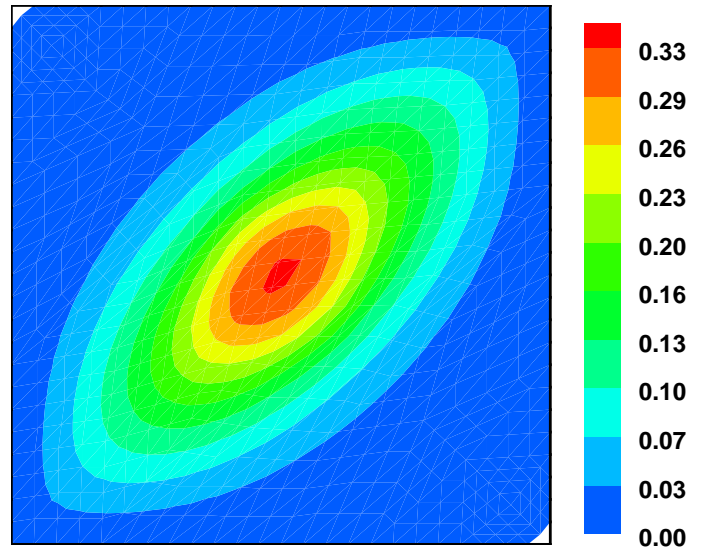

(b) Anisotropic mesh: $\mathbf{v}=(0,0)$ and $\alpha=0$

Figure 19. Test problem \#2: The left figure shows the concentration profile based on the background mesh, while the right figure shows the concentration profile using the anisotropic triangulation.

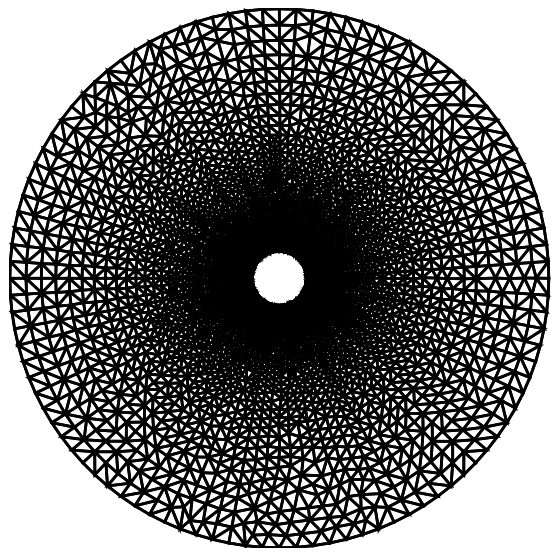

(a) Background mesh: $N v=5079$ and $N e l e=9918$

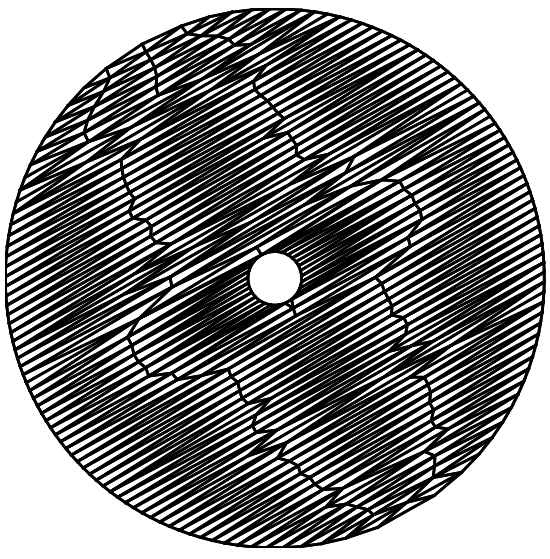

(b) Anisotropic mesh: $N v=297$ and Nele $=436$

Figure 20. Test problem \#3: The left figure shows the background mesh and the right figure shows the anisotropic triangulation obtained using BAMG for all the four cases. 

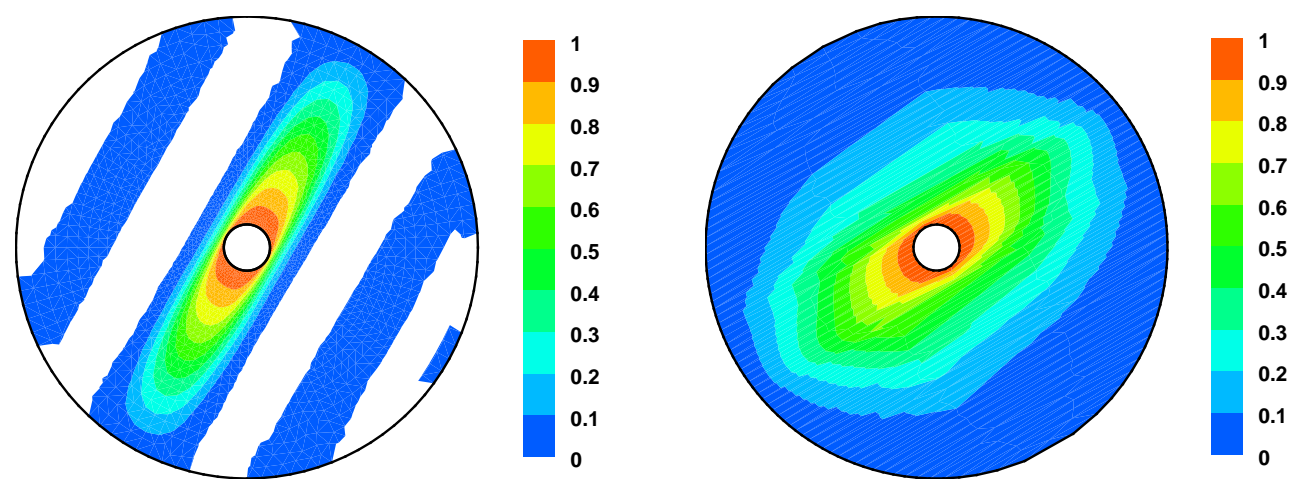

(a) Background mesh: $\mathbf{v}=(0,0)$ and $\alpha=0$

(b) Anisotropic mesh: $\mathbf{v}=(0,0)$ and $\alpha=0$
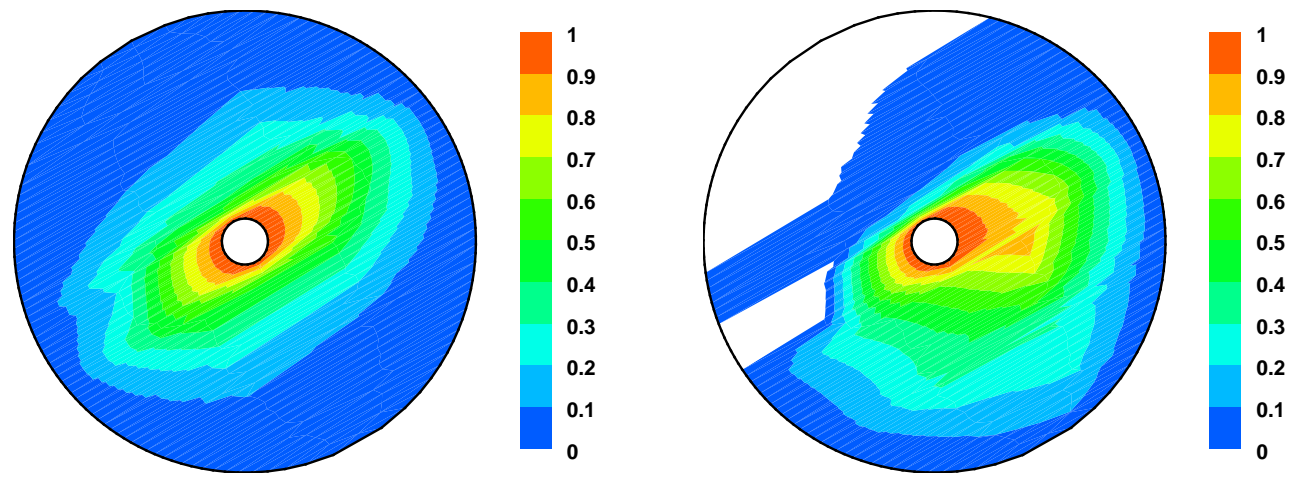

(c) Anisotropic mesh: $\mathbf{v}=(1.5,1.0)$ and $\alpha=1.0(\mathrm{~d})$ Anisotropic mesh: $\mathbf{v}=(5.0,0.5)$ and $\alpha=1.0$

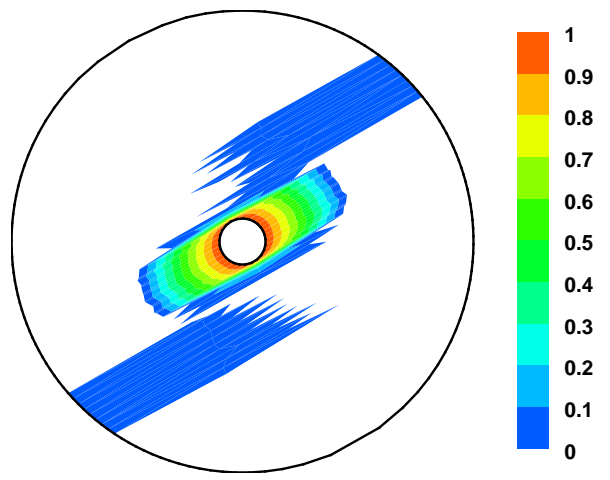

(e) Anisotropic mesh: $\mathbf{v}=(0,0)$ and $\alpha=1000$

Figure 21. TEST PROBlem \#3: This figure shows the concentration profiles for four different cases based on the background mesh and anisotropic meshes shown in Figure 20. 

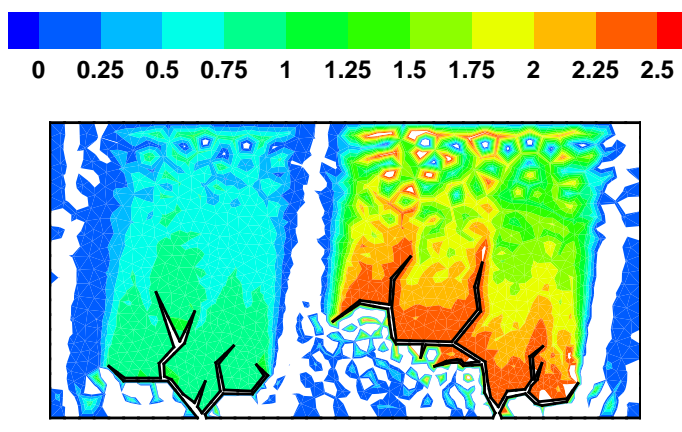

(a) Delaunay mesh: $N v=1564$ and Nele $=2826$
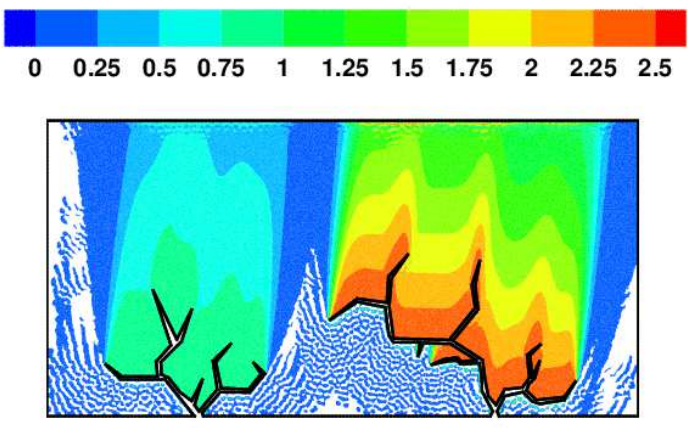

(c) Delaunay mesh: $N v=18372$ and Nele $=35665$
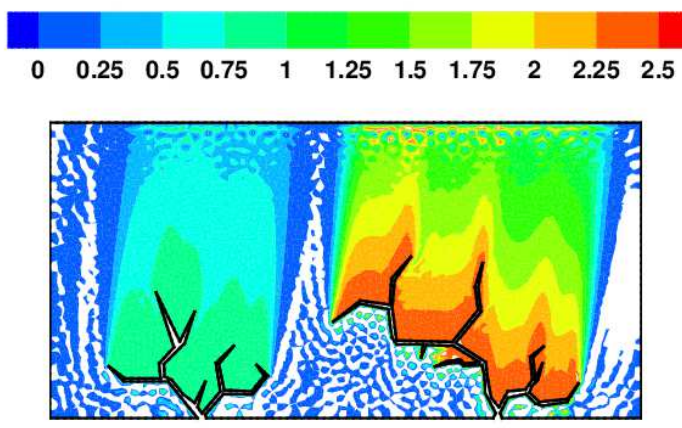

(b) Delaunay mesh: $N v=5090$ and Nele $=9620$
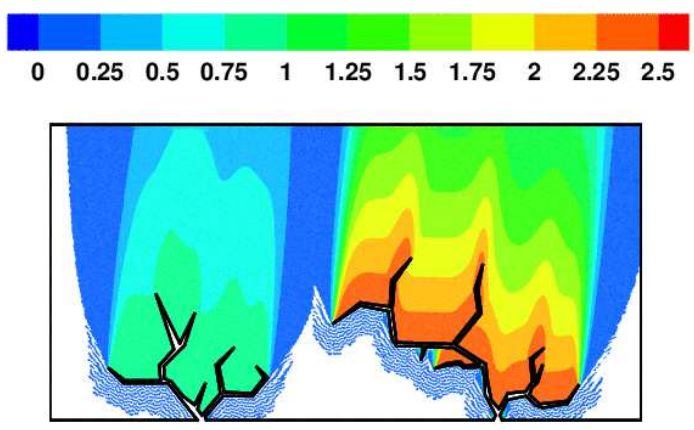

(d) Delaunay mesh: $N v=69995$ and $N e l e=137881$

Figure 22. Issues with traditional mesh Refinement: Concentration profiles for the fracture domain when $\mathbf{v}=(0.1,1.0)$ and $\alpha=1.0$. The white region in the figures shows the area in which the numerical simulation has violated the $\mathrm{NC}$ and maximum constraint. 


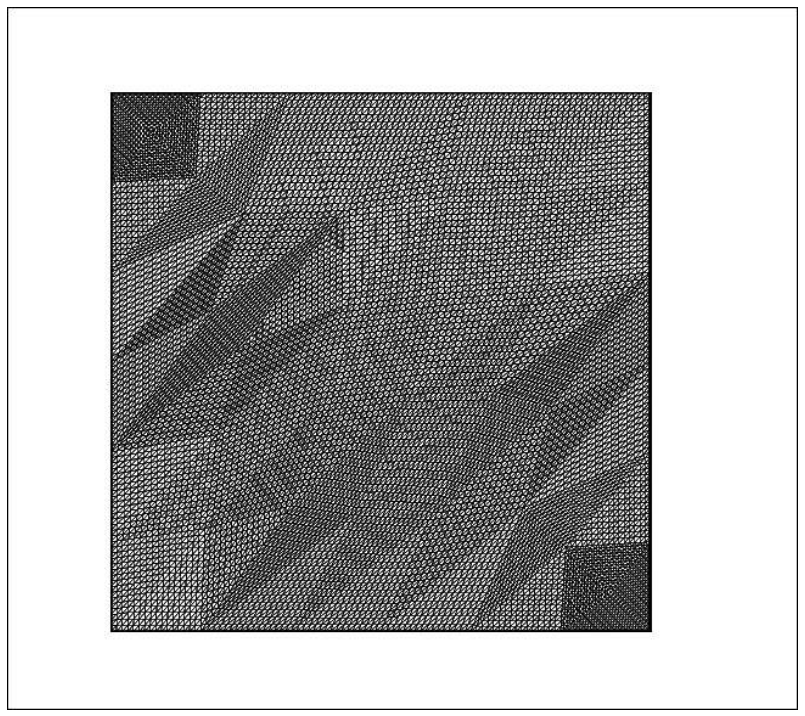

(a) Anisotropic mesh: $N v=8897$ and $N e l e=17408$

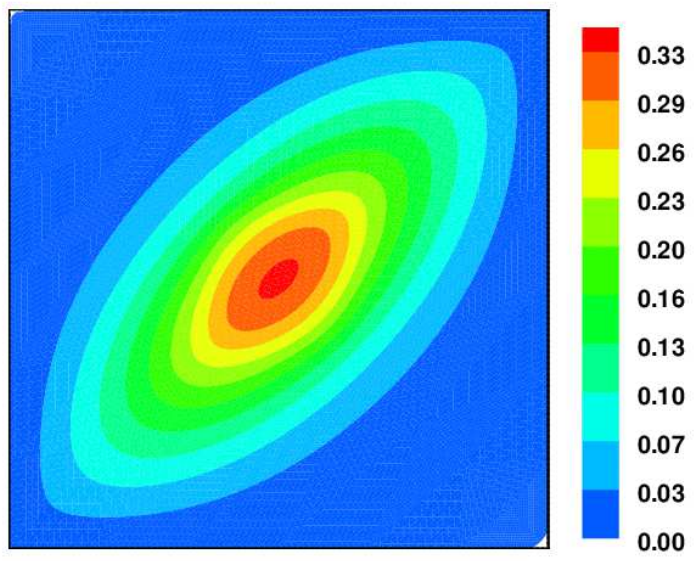

(b) Pure anisotropic diffusion: Concentration profile

Figure 23. Issues With TRADitional MESH REFInEMEnt: The left figure shows the anisotropic mesh obtained using the traditional mesh refinement procedure on the anisotropic triangulation given in Figure 18. The right figure shows the concentration profile obtained using this refined mesh. It should be noted that this $h$-refined DMP-based mesh is interiorly connected. Hence, it satisfies $\operatorname{DSMP}_{\boldsymbol{K}}$.

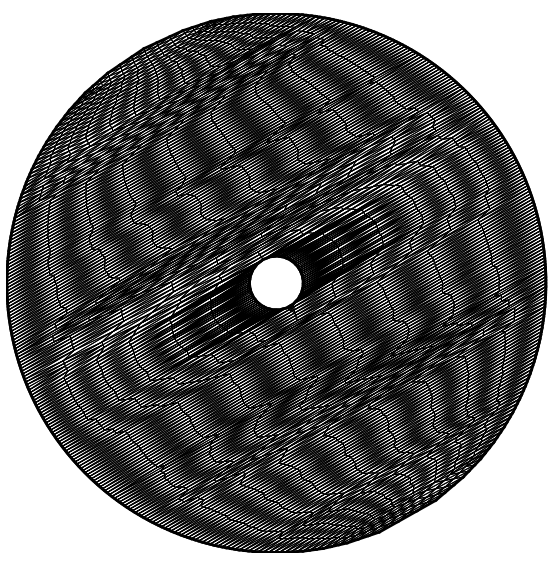

(a) Anisotropic mesh: $N v=2199$ and $N e l e=3924$

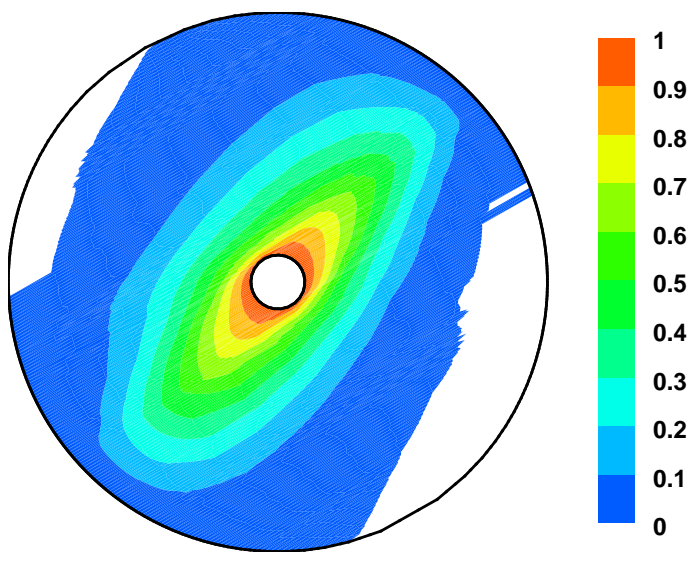

(b) Pure anisotropic diffusion: Concentration profile

Figure 24. Issues With traditional MESH REFinEmEnt: The left figure shows the anisotropic mesh obtained using the traditional mesh refinement procedure on the anisotropic triangulation given in Figure 20. The right figure shows the concentration profile obtained using this refined mesh. 


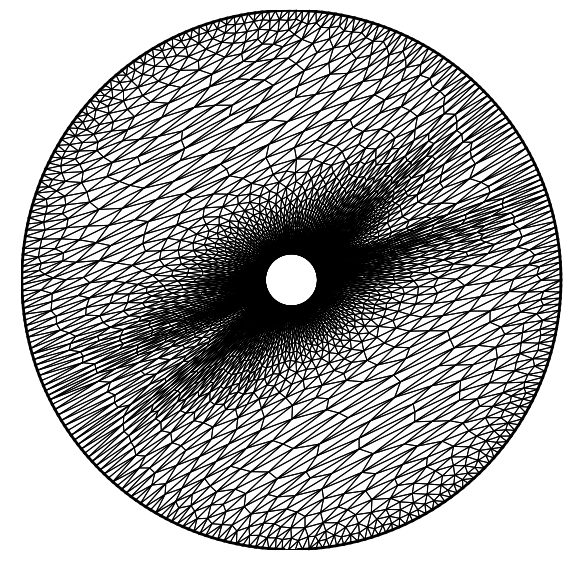

(a) Anisotropic mesh: $N v=2647$ and Nele $=4789$

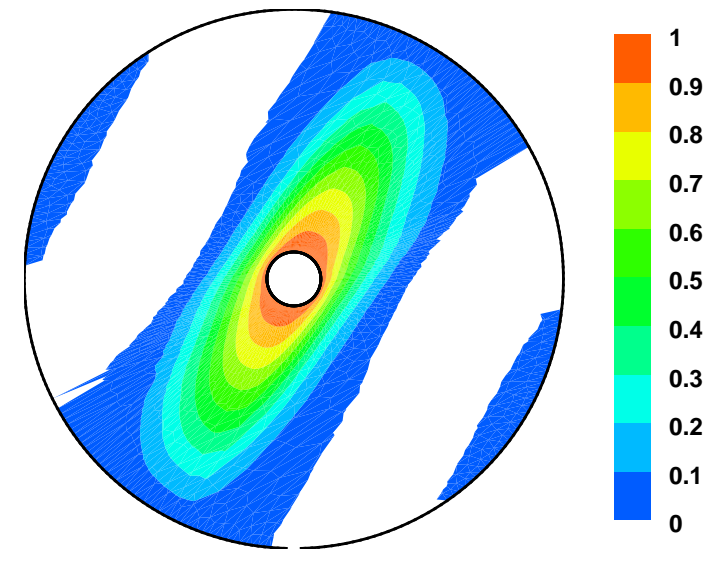

(b) Pure anisotropic diffusion: Concentration profile

Figure 25. Issues With nON-Traditional mesh REFinement: The left figure shows a refined anisotropic mesh obtained using the non-traditional approach. The right figure shows the concentration profile obtained using this refined mesh. It should be pointed out that the mesh obtained using this procedure did not converge in MaxIters $=100$. Hence, as a result, it violates $\mathrm{NC}$ and DMPs. 


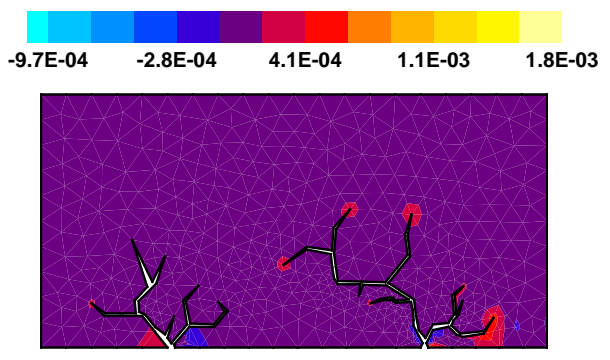

(a) Test problem \#1: $\mathbf{v}=(0,0)$ and $\alpha=0$

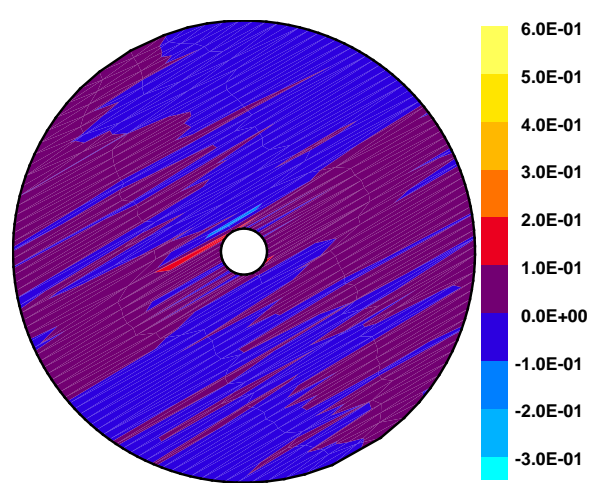

(c) Test problem \#3: $\mathbf{v}=(0,0)$ and $\alpha=0$

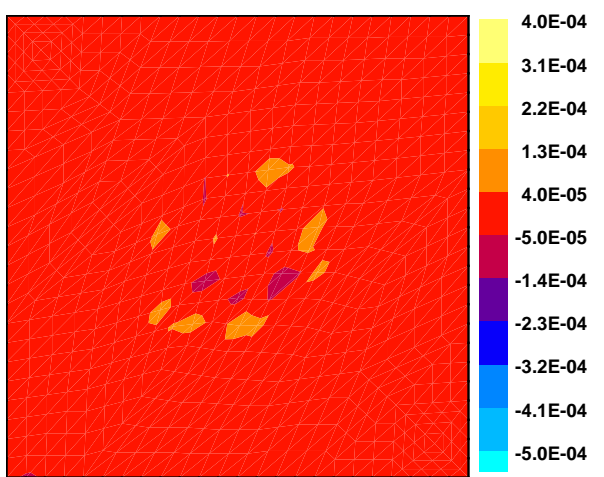

(b) Test problem \#2: $\mathbf{v}=(0,0)$ and $\alpha=0$

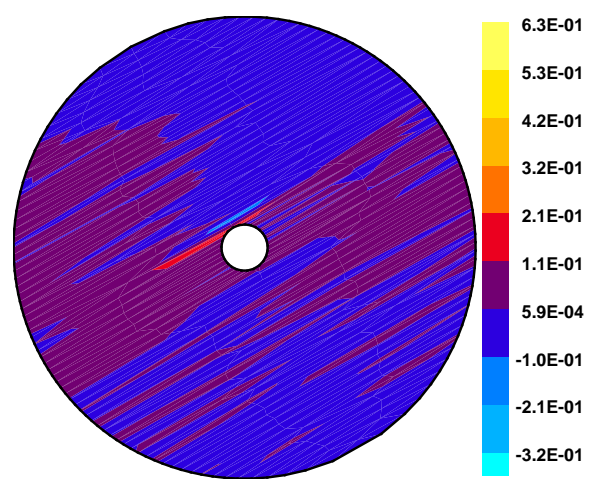

(d) Test problem \#3: $\mathbf{v}=(1.5,1.0)$ and $\alpha=1.0$

Figure 26. Local SPECIES BALANCE ERRORs: The figures show the errors incurred in satisfying local species balance for various test problems on coarse meshes. 